\title{
British Thoracic Society guideline for respiratory management of children with neuromuscular weakness
}

\author{
Jeremy Hull, ${ }^{1}$ Roona Aniapravan, ${ }^{2}$ Elaine Chan, ${ }^{3}$ Michelle Chatwin ${ }^{4}$ Julian Forton, ${ }^{5}$ \\ Jayne Gallagher, ${ }^{1}$ Neil Gibson, ${ }^{6}$ Jill Gordon, ${ }^{7}$ Imelda Hughes, ${ }^{3}$ Renee McCulloch, ${ }^{8}$ \\ Robert Ross Russell, ${ }^{2}$ Anita Simonds ${ }^{4}$
}

\begin{abstract}
- Additional appendices are published online only. To view these files please visit the journal online (http://thorax.bmj. com/content/67/S1.toc)
\end{abstract}

1 Department of Paediatrics, Oxford University Hospitals NHS Trust, Oxford, UK

${ }^{2}$ Department of Paediatrics, Cambridge University Hospitals NHS Foundation Trust, Cambridge, UK

${ }^{3}$ Royal Manchester Children's Hospital, Manchester, UK

${ }^{4}$ National Institute of Health Research Respiratory Biomedical Research Unit, Royal Brompton and Harefield NHS Foundation Trust, UK

${ }^{5}$ University Hospital of Wales, Cardiff, UK

${ }^{6}$ Royal Hospital for Sick Children, Glasgow, UK

${ }^{7}$ Child Development Centre Suffolk Community Healthcare, UK

${ }^{8}$ Great Ormond St Hospital, London, UK

\section{Correspondence to}

Jeremy Hull, Level 2, Children's Hospital, John Radcliffe

Hospital, Oxford, OX3 9DU, UK; jeremy.hull@ouh.nhs.uk

Received 26 March 2012 Accepted 5 April 2012

\section{SUMMARY OF RECOMMENDATIONS Identifying children at risk of respiratory complications}

- Clinical assessment of respiratory health should be part of every medical consultation for children with neuromuscular weakness (NMW) and should be directed towards identifying progressive muscle weakness, ability to cope with respiratory infection, aspiration, progression of scoliosis and sleep-disordered breathing. [D]

- Ulna length or armspan should be used to predict lung function in children with neuromuscular disease whose height cannot be accurately measured. [B]

- Vital capacity should be measured in all patients with neuromuscular disease who are capable of performing spirometry as part of the respiratory assessment. [C]

- Cough peak flow should be used as part of the assessment of effective secretion clearance in children with neuromuscular disease over the age of 12 years.

- Assessment for sleep-disordered breathing should be carried out no less than annually for children with neuromuscular disease who have a vital capacity of $<60 \%$ predicted and for children who have become non-ambulant because of progressive muscle weakness or who never attain the ability to walk. [D]

- Assessment for sleep-disordered breathing should be carried out no less than annually for all infants with weakness, children with NMW who have symptoms of obstructive sleep apnoea or hypoventilation, children with clinically apparent diaphragmatic weakness and children with rigid spine syndromes. [ $\sqrt{ }]$

- In young children whose rate of disease progression is uncertain, or in older children who have shown a clinical deterioration or who are suffering with repeated infections, or who develop symptoms of sleep-disordered breathing, sleep assessment may need to be more frequent than once a year. $[\sqrt{ }]$

- All children with abnormal overnight oximetry should undergo more detailed sleep monitoring with at least oxycapnography. [ $\sqrt{ }]$

-When there is doubt about the cause of sleepdisordered breathing, overnight polysomnography or sleep polygraphy should be performed. [ $\sqrt{ }]$

- Portable overnight oxycapnography or polygraphy in the home may be the most appropriate option for some patients. [ $\sqrt{ }]$
- Children with neuromuscular disease with a history of swallowing difficulties should have a feeding assessment by a speech and language therapist including a video fluoroscopy swallow assessment if the swallow is thought to be unsafe. $[\sqrt{ }]$

\section{Airway clearance and respiratory muscle training}

- Children with ineffective cough (including children over 12 years of age with cough peak flow $<270$ litres/min), particularly if they have had episodes of deterioration with respiratory infection, should be taught augmented cough techniques. [C]

- Manual cough assist and air-stacking methods to achieve maximum insufflation capacity are effective methods of improving cough efficiency and should be used when appropriate. [C]

- Mechanical insufflation/exsufflation (MI-E) should be considered in very weak children, those with loss of bulbar function, and those who cannot cooperate with manual cough assist or air-stacking or in whom these methods are not effective. [C]

- Oscillatory techniques such as high-frequency chest wall oscillation and intrapulmonary percussive ventilation should be considered in children who have difficulty mobilising secretions or who have persistent atelectasis, despite use of other airway clearance techniques. [D]

- Airway clearance techniques should be used during respiratory infection when oxygen saturation levels fall below $95 \%$ while the child is breathing room air. If the techniques being used fail to result in an increase in oxygen saturation to $95 \%$ or above, different methods of airway clearance should be used. This may require attendance at hospital for treatment. [D]

-MI-E should be available in the acute setting in all hospitals that treat neuromuscular patients as an alternative method of airway clearance with the purpose of preventing deterioration and the need for intubation and mechanical ventilation. [D]

-Nebulised normal saline may be considered in children who have continued tenacious secretions. [ $\sqrt{ }]$

- Humidification should be considered in children who use non-invasive ventilation (NIV) and who have tenacious airway secretions. Care should be taken to ensure that use of humidification does not result in a troublesome increase in oral secretions. $[\sqrt{ }]$

-When using sputum mobilising techniques, appropriate emergency equipment (eg, resuscitation bag 
and suction) should be available in case of mobilising large mucus plugs into the central airways where they may result in airways obstruction. [ $\sqrt{ }]$

- Children who use regular night-time or diurnal NIV should use their ventilator to support deep breathing during airway clearance treatments. Use of NIV during airway clearance sessions can help prevent respiratory muscle fatigue. [ $\sqrt{ }]$

- Children who use MI-E for airway clearance should be given long enough periods of rest during treatment sessions to prevent respiratory muscle fatigue due to coughing. [ $\mathrm{J}$ ]

- At the end of a treatment session with MI-E it is important to complete the session with an insufflation to leave the child with an appropriate functional residual capacity. [ $\sqrt{ }]$

\section{Assisted ventilation}

- Children with NMW resulting in symptomatic nocturnal hypoventilation or daytime hypercapnia should be supported with NIV. [C]

- A non-invasive approach should be considered in children needing daytime ventilation. [D]

- Clinical teams caring for children using home ventilators should become familiar with a small number of machines. For most children pressure-targeted machines work well and are simple to use. [ $\sqrt{ }]$

- Ventilation modes with fixed inspiratory times are usually the most appropriate for use in young or very weak children. [ $/]$

- For older teenage children who need to use mouthpiece ventilation during the day, and/or who use air-stacking as a way of assisting cough, volume-targeted or hybrid ventilators may be preferred. $[\sqrt{ }]$

-When full face masks are used, anti-asphyxia valves should be fitted to allow room air breathing in case of ventilator failure. The risks of the child vomiting and aspirating should be considered, particularly if the child is unable to remove the mask on their own. [ $\sqrt{ }]$

- Children needing ventilatory support for more than $16 \mathrm{~h}$ per day should be provided with two ventilators in case of equipment failure. $[\sqrt{ }]$

- Once a child has become established on NIV, a sleep polygraphy or oxycapnography should be carried out to check that it has effectively abolished sleep-associated hypoventilation. Ventilator settings should be adjusted and rechecked as necessary. [D]

- Children supported by NIV should be assessed regularly with repeat sleep studies to ensure continued effectiveness of NIV at preventing hypoventilation. The frequency of review will vary according to clinical circumstances, but should not be less than every 12 months. [D]

- Assessment for skin injury and facial flattening should be carried out regularly in children using NIV and the mask interface adjusted as necessary to minimise these complications. [ $\sqrt{ }]$

- Tracheostomy tubes should be carefully sized and sited to ensure the tip of the tube does not abut the tracheal wall. [V]

- Family and child preference should be taken into account when considering tracheostomy to facilitate diurnal ventilation. $[\sqrt{ }]$

- Oxygen alone should not be used to correct hypoxaemia caused by hypoventilation in patients with neuromuscular disease. $[\sqrt{ }]$

- NIV should be the first-line treatment for children with NMW in acute respiratory failure. [D]

- Intensive care units caring for children with NMW should be aware of appropriate extubation criteria. These should include the presence of only minimal airway secretions, use of effective airway clearance methods (such as MI-E devices) and oxygen saturation more than $94 \%$ without supplemental oxygen for more than $12 \mathrm{~h}$. Continuous NIV should be used immediately after extubation. [ J]

\section{Planning for surgical procedures}

- Surgery in children with NMW should take place in units with experienced paediatric surgeons, anaesthetists and physiotherapists, and where there are facilities for paediatric intensive care and NIV. [D]

- Extubation protocols for high-risk children (those with vital capacity $<60 \%$ predicted and/or ineffective cough and/or already use NIV for hypoventilation) should include effective airway clearance techniques and immediate use of noninvasive ventilation following extubation. [ J]

- Children with NMW who require surgery (including scoliosis surgery) should be assessed by a multidisciplinary team prior to any intervention. $[\sqrt{ }]$

\section{Scoliosis}

- The effect of wearing a spinal brace on respiratory function should be assessed and weighed against the limited evidence of benefit in terms of affecting final scoliosis severity. [D]

- The primary consideration when planning surgery for children with scoliosis associated with NMW should be comfort and quality of life. [ $\sqrt{ }]$

\section{Other problems and interventions that impact on respiratory health}

- Videofluoroscopy and feeding advice from a specialist therapist is indicated in children with a history of recurrent chest infection or swallowing difficulties. [ $\sqrt{ }]$

- A problem-orientated approach to nutrition should aim to minimise risk of aspiration, optimise nutritional status, promote comfort and balance the positive social consequences of continued oral feeding. $[\sqrt{ }]$

\section{Transition to adult care}

- A key worker can act as a valuable advocate, a source of knowledge and provide support for young people with neuromuscular disease and their families during the period of transition from paediatric to adult services. [ $\sqrt{ }]$

\section{Quality of life and palliative care}

- Evaluating the child and parent or carer using multidimensional health-related quality of life assessment tools should be the standard for routine assessment in clinical practice and future clinical trials. [D]

- International standardised disease-specific tools for children and young people with neuromuscular disorders should be used if available to evaluate clinical interventions and patientrelated outcome measures with respect to quality of life. [D]

- Assisting patients, parents and carers to make informed choices that are consistent with their own values and preferences requires physicians to engage patients and their parents and carers in a process of mutual participation in decision-making with full disclosure of all information in a sensitive and timely fashion. [D]

-Open discussion across the multidisciplinary team regarding the type and duration of specific interventions encourages transparency and shared decision-making. [ $\sqrt{ }]$

- Benefit and burden of all interventions must be considered with respect to impact on mental health of patients and their families. [D] 
- Acknowledgement of stress factors and mental health issues, requirement for wider support through organizations, and practical and focused individual care are needed for patients and their parents or carers. [D]

- Routine pain evaluation should be part of standard clinical assessment in all children and young people with neuromuscular disorders. [D]

- Attention to the wider physical impact of chronic illness through vigilant symptom management is required. [D]

-Dyspnoea is a subjective feeling, which can respond to non-drug measures and treatment directed at the cause. $[\sqrt{ }]$

- Low-dose opioids may be used to manage distressing breathlessness even when active treatment is pursued. With slow titration of opioids, respiratory depression is extremely unlikely. [ $\sqrt{ }]$

- In the terminal phase of respiratory failure, symptoms must be reassessed frequently and can be effectively managed by a multimodal approach, including careful titration of opioids and psychotropic agents. $[\sqrt{ }]$

- Advance care planning should be an integral part of the active management of children and young people with neuromuscular disorders. Advance care plans can be used as a vehicle for information exchange and considered decision-making. [D]

- Patients and families need to have ownership of the advance care plan and be educated as to its uses. [D]

- Advance care plans should be reviewed by the multidisciplinary team on a regular basis. [ $\sqrt{ }]$

- Families need access to skilled experts for multidimensional coordinated palliative care support, providing regular review of their needs at various stages in their condition. [D]

- Generic palliative care skills should be cascaded to other professionals providing neuromuscular services. [ $\sqrt{ }]$

\section{Special considerations}

- Carers and parents of children with severe NMW, including all those using NIV and all those with tracheostomy, should have basic life support training. $[\sqrt{ }]$

-Written plans for the management of acute exacerbations, which include details of effective airway clearance methods and ventilator settings when appropriate, and contact details of relevant healthcare professionals are recommended. [ $\sqrt{ }]$

\section{INTRODUCTION}

\section{Clinical context and need for a guideline}

Neuromuscular diseases as a group are relatively common with a prevalence of about 1 in 3000 . The majority of these conditions are genetic and become clinically apparent during childhood. The likelihood of respiratory impairment varies greatly among the different conditions; with some notable exceptions, it is more likely in children with more severe global weakness. Acute respiratory failure associated with respiratory infection is the most frequent reason for unplanned hospital admission, and chronic respiratory failure is a frequent cause of death. With appropriate intervention, the incidence of unplanned hospital admission can be reduced and life expectancy can be improved. This guideline attempts to summarise the available evidence in this field and to provide recommendations that will aid the healthcare professional in delivering good quality patient care.

There are a number of excellent disease-specific guidelines and consensus statements on neuromuscular diseases, but none which focus specifically on respiratory management. Many of the principles of respiratory management are not disease specific and the objective of this guideline is to provide recommendations that can be applied to all children with neuromuscular weakness (NMW). The evidence for much of current practice is weak and is based largely on observational studies. The Guideline Committee attempted to identify and summarise the existing evidence, and when that is lacking, provide expert consensus opinion.

\section{Guideline Committee members}

The full list of Guideline Committee members is given in appendix 1. The members adhered to the British Thoracic Society (BTS) policy for the declaration of interests, and if appropriate, specific interests are declared in appendix 1.

\section{Target audience for the guideline}

This guideline is aimed primarily at healthcare practitioners in the UK who care for children with NMW, including doctors, nurses, physiotherapists and other healthcare professionals.

\section{Scope of the guideline}

The guideline starts with a background overview of respiratory problems in children with NMW. A brief summary of the conditions covered by the guideline is provided in appendix 2 . The respiratory management of these children is then covered in the following sections:

- Identifying children at risk of respiratory complications

- Airway clearance and respiratory muscle training

- Assisted ventilation

- Planning for surgical procedures

- Scoliosis

- Other problems and interventions that impact on respiratory health

- Transition to adult care

- Quality of life and palliative care

- Special considerations

Details of individual studies that form part of the evidence behind the recommendations are available in the evidence tables (online appendix S1).

\section{Areas not covered by the guideline}

The following areas fall outside the scope of the guideline:

- Non-respiratory aspects of management of children with neuromuscular disease

- Detailed anaesthetic management of children with neuromuscular disease

- Adults with neuromuscular disease

- Children with cerebral palsy

- Children with myasthenia gravis

- Children with Guillain-Barré syndrome

\section{Methodology}

This guideline is based on the best available evidence. The methodology used to write the guideline adheres strictly to the criteria set out by the Appraisal of Guidelines for Research and Evaluation (AGREE) collaboration (available online at http://www.agreecollaboration.org/1/agreeguide/). The Committee is grateful for the assistance of Dr Ingrid Du Rand who provided advice on guideline methodology.

\section{Clinical questions and literature search}

Clinical questions were gathered in the PICOT format to define the scope of the guideline and inform the literature search.

Systematic electronic database searches were conducted to identify potentially relevant studies for inclusion in the guideline. For each topic area the following databases were searched: Ovid MEDLINE (including MEDLINE In Process), Ovid 
EMBASE, EMSCO CINAHL, Ovid PsycINFO and the Cochrane Library (including the Cochrane Database of Systematic Reviews, the Database of Abstracts of Reviews of Effects, and the Cochrane Central Register of Controlled Trials).

The searches were first run in March 2010 and were updated in May 2011. Searches included a combination of indexed terms and free text terms. Searches were not limited to studies on children. The searches identified a total of 4683 potential papers. The search strategy is available in online appendix S2.

\section{Appraisal of the literature}

Appraisal was performed using the criteria stipulated by the AGREE collaboration. Each paper was appraised by a pair of reviewers. One individual $(\mathrm{JH})$ read the title and abstract of each article retrieved by the literature searches and decided whether the paper was definitely relevant, possibly relevant or not relevant to the project. The following criteria were formulated for categorising the abstracts into these three groups were:

- Whether the study addressed the clinical question.

- Whether the appropriate study type was used to produce the best evidence to answer the clinical question.

- The abstract was in English.

- Abstracts were not rejected on the basis of the journal of publication, the country in which the research was performed or published, or the date of publication.

- Abstracts were not rejected if they dealt only with adults provided that the topic was relevant to children with NMW.

The full paper was obtained for all relevant or possibly relevant abstracts and allocated to the relevant section(s). The first screening process identified 686 of the 4683 reference abstracts to be definitely or possibly relevant to the guideline. Two guideline reviewers independently reviewed the abstracts to identify papers to be appraised for the guideline.

A total of 177 papers were critically appraised. The two leads for each section independently appraised each paper assigned to them using the Scottish Intercollegiate Guidelines Network (SIGN) critical appraisal checklists. An evidence grade was assigned to each study using the SIGN grading system (see table 1) and is shown in the evidence tables (online appendix S1).

\section{Considered judgement and grading of the evidence}

The Guideline Committee used evidence tables to judge the body of evidence and grade recommendations for this guideline. Evidence tables are available in online appendix S1.

Table 1 Revised grading system for recommendations in evidencebased guidelines

\begin{tabular}{ll}
\hline Grade & Evidence \\
\hline $1++$ & $\begin{array}{l}\text { High-quality meta-analyses, systematic reviews of RCTs or RCTs } \\
\text { with a very low risk of bias }\end{array}$ \\
$1+$ & $\begin{array}{l}\text { Well conducted meta-analyses, systematic reviews of RCTs or RCTs } \\
\text { with a low risk of bias }\end{array}$ \\
$1-$ & $\begin{array}{l}\text { Meta-analyses, systematic reviews or RCTs or RCTs with a high risk } \\
\text { of bias } \\
\text { High-quality systematic reviews of case-control or cohort studies or } \\
\text { high-quality case-control or cohort studies with a very low risk of } \\
\text { confounding, bias or chance, and a high probability that the }\end{array}$ \\
& $\begin{array}{l}\text { relationship is causal } \\
\text { Well conducted case-control or cohort studies with a low risk of } \\
\text { confounding, bias or chance, and a moderate probability that the } \\
\text { relationship is causal } \\
\text { Case-control or cohort studies with a high risk of confounding, bias } \\
\text { or chance and a significant risk that the relationship is not causal }\end{array}$ \\
$3-$ & $\begin{array}{l}\text { Non-analytic studies, for example, case reports, case series } \\
\text { Expert opinion }\end{array}$
\end{tabular}

RCT, randomised controlled trial.
When evidence was lacking to answer the formulated clinical questions, expert opinions were obtained for formal consensus statements using the Delphi method.

The following factors were considered when grading the recommendations:

- The available volume of the body evidence.

- How applicable the obtained evidence was in making recommendations for the defined target audience of this guideline.

- Whether the evidence was generalisable to the target population for the guideline.

- Whether there was a clear consistency in the evidence obtained to support recommendations.

- What the implications of recommendations will be on clinical practice in terms of recourses and skilled expertise.

- Cost effectiveness was not reviewed in detail because in-depth economic analysis of the recommendations falls beyond the scope of this guideline.

Recommendations were graded from $\mathrm{A}$ to $\mathrm{D}$ as indicated by the strength of the evidence as listed in table 2. Important practical points lacking any research evidence were highlighted as 'Good practice points'.

\section{Drafting the guideline}

The Guideline Committee corresponded regularly by email and meetings of the full group were held in March 2010, November 2010, March 2011 and May 2011. A draft guideline document was circulated to all the relevant stakeholders (appendix 3) for consultation in October/November 2011 and the draft guideline was made available for public consultation via the BTS website in November 2011. The BTS Standards of Care Committee reviewed the draft guideline in November 2011. The guideline was revised, taking account of comments from the Committee, stakeholders and feedback from public consultation, and submitted for publication in March 2012.

The guideline will be reviewed in 2016.

\section{Audit and research recommendations}

\section{Audit}

- Local databases should be kept of children with NMW, which should include whether a respiratory assessment has been made and the planned frequency of future assessments.

- Audits of non-elective hospital admissions for children with NMW would provide important information when planning the need for respiratory support services.

Table 2 Grades of recommendation

Grade Type of evidence

A At least one meta-analysis, systematic review, or RCT rated as $1++$ and directly applicable to the target population or

A systematic review of RCTs or a body of evidence consisting principally of studies rated as $1+$ directly applicable to the target population and demonstrating overall consistency of results

B A body of evidence including studies rated as $2++$ directly applicable to the target population and demonstrating overall consistency of results or

Extrapolated evidence from studies rated as $1++$ or $1+$

C A body of evidence including studies rated as $2+$ directly applicable to the target population and demonstrating overall consistency of results or Extrapolated evidence from studies rated as $2++$

D Evidence level 3 or 4 or

Extrapolated evidence from studies rated as $2+$

$\sqrt{ } \quad$ Important practical points for which there is no research evidence, nor is there likely to be any research evidence. The Guideline Committee wishes to emphasise these as good practice points 


\section{Research}

- A randomised controlled trial of MI-E compared with other methods of assisted cough in children, used at home to prevent acute respiratory deterioration.

- A comparative hospital-based study to evaluate the efficacy of different airway clearance techniques during acute exacerbations associated with respiratory infection. Possible outcomes would include immediate effects on assessment of mobilisation of secretions and oxygen saturation, and on avoidance of intubation and length of hospital stay.

- Prospective cohort study to evaluate methods to assess cough effectiveness in children and their association with respiratory morbidity.

- Prospective randomised control trial to evaluate the effectiveness of prophylactic antibiotics in the prevention of respiratory exacerbations in children with NMW.

- Further development of disease-specific quality of life (QOL) tools is required for children with neuromuscular disease.

- Baseline studies regarding the incidence, prevalence, impact and management of dyspnoea in paediatric neuromuscular disorders.

- Long-term studies to determine whether respiratory muscle training improves clinical outcomes.

- Patient-related outcome measures are required to set standards, shape future services and facilitate integration between palliative care and neuromuscular services.

- Further evaluation to develop a standardised approach to transition for young people with neuromuscular disease is required.

\section{RESPIRATORY COMPLICATIONS OF NMW}

NMW can compromise the gas exchange and pump functions of the respiratory system, maintenance of upper airway muscle tone, airway protection, efficiency of secretion clearance and spinal support. The respiratory consequences are hypoventilation, upper airway obstruction, aspiration lung disease, secretion retention and lower airway infection, and the mechanical effects of progressive scoliosis. These complications are not mutually exclusive and often coexist, leading to progressive respiratory insufficiency. Respiratory failure is the most common cause of mortality in patients with neuromuscular disease. ${ }^{12}$ The likelihood of respiratory morbidity for most children is dependent on the severity of their weakness, although there are exceptions, particularly those children with rigid spine syndromes and congenital myasthenic syndromes. The age of onset of respiratory problems varies with the underlying condition, as indicated in table 3.

\section{Pulmonary function}

Lung function testing in children with NMW typically shows a restrictive pattern with diminished vital capacity, total lung capacity and functional residual capacity, and with a relative preservation of forced expiratory volume in $1 \mathrm{~s}\left(\mathrm{FEV}_{1}\right) /$ forced vital capacity (FVC) ratio. Scoliosis can worsen the restrictive defect. $^{3}$ Lung compliance may be diminished by microatelectasis. ${ }^{4}$ Chest wall compliance may reduce as time progresses, independent of scoliosis, ${ }^{6}$ attributed to fibrotic changes of the dystrophic chest wall muscles and shortening of the un-stretched tissues. ${ }^{78}$

\section{Retention of airway secretions}

The transport of secretions from the peripheral airways to the main airways is mainly carried out by ciliary activity. In normal conditions, it is estimated that the amount of secretions reaching the trachea is between 10 and $100 \mathrm{ml} /$ day. ${ }^{9}$ Removal of airway secretions from the trachea requires a huff or cough. Effective coughing involves three main components ${ }^{10}$ :

- A deep inspiration up to $95 \%$ of total lung capacity.

- Glottic closure (which requires intact bulbar function) and short pause to allow distribution of air past secretions, including via collateral ventilation.

- Effective contraction of the expiratory muscles (abdominal and intercostal muscles) to generate high intrapleural pressures and subsequent rapid expiratory flow rates.

The sequence of phases in a cough manoeuvre can be classified as inspiratory, compressive and expiratory. ${ }^{11}$ The result is fastmoving air within the large airways, sufficient to pick up airway secretions and expel them from the airway. Respiratory infections are characterised by increased airway secretions, as a result of increased mucous production and the presence of inflammatory cells, cell debris and bacteria. Ciliary activity is hampered by airway inflammation, and coughing to expel airway secretions becomes more important. In children with NMW, reduction in inspiratory, glottic and expiratory muscle strength results in reduction of cough effectiveness. ${ }^{12} 13$ Weakness may worsen during acute infection, reducing cough effectiveness at a critical time. Retained airway secretions cause atelectasis which results in ventilation perfusion mismatch and hypoxaemia, as well as reduced lung compliance. Without assistance, respiratory muscles may tire leading to respiratory failure. Most respiratory infections in children with NMW, as in other children, will be viral.

\section{Swallowing dysfunction, loss of airway protection and aspiration lung disease}

The prevalence of swallowing dysfunction tends to increase as muscle weakness progresses; in some conditions such as spinal muscular atrophy (SMA) type 1 and severe forms of nemaline myopathy, it may present from early infancy. Difficulties with swallowing can lead to under-nutrition or risk of aspiration. Aspiration lung disease is common in children with NMW, resulting from loss of control of the larynx and pharynx, and ineffective cough. Aspirated material includes saliva, mouth organisms and possibly food in children who are orally fed. Gastric contents may be aspirated in children who have gastrooesophageal reflux. Aspiration causes inflammation within the lungs leading to airways obstruction and to worsening restrictive lung disease. Recurrent aspiration can lead to bronchiectasis and pulmonary fibrosis. The prevalence of gastrooesophageal reflux among children with neuromuscular conditions is not known, although clinical experience suggests that it is a common problem in children with NMW, especially non-mobile children.

\section{Impact of nutritional status}

Assessment and management of nutritional status can be challenging in children with neuromuscular disease. Patients are at risk of malnutrition (because of feeding difficulties, dysphagia and gastro-oesophageal reflux) and obesity. ${ }^{14}$ In one survey of boys with Duchenne muscular dystrophy (DMD) ${ }^{15}$ approximately $50 \%$ of boys were overweight at age 13 years; in older boys with more severe weakness, under-nutrition became more common and approximately 50\% of boys between 14 and 18 years of age were said to be undernourished. Children with severe weakness, such as those with severe forms of SMA, frequently require nasogastric or gastrostomy feeding to maintain adequate nutrition. Assessment of nutritional status in children with NMW is complicated by progressive loss of muscle 
Table 3 Respiratory features of neuromuscular diseases

\begin{tabular}{|c|c|c|c|c|c|}
\hline Condition & Respiratory failure & $\begin{array}{l}\text { Secretion } \\
\text { clearance difficulty }\end{array}$ & Recurrent pneumonia & Progression & Disease-specific features \\
\hline \multicolumn{6}{|l|}{ SMA } \\
\hline Type 1 & All by 2 years & Marked & All & Rapid & $\begin{array}{l}\text { All require full-time } \\
\text { respiratory support }\end{array}$ \\
\hline Type 2 & $\sim 40 \%$ in childhood & Early & $\sim 25 \%$ in first 5 years & Slow & \\
\hline Type 3 & Rare in childhood & Rare in childhood & Rare in childhood & Slow & \\
\hline $\begin{array}{l}\text { SMA with respiratory } \\
\text { distress type } 1\end{array}$ & All by 6 months & Marked & All & $\begin{array}{l}\text { Rapid in first } \\
\text { year, then slows. }\end{array}$ & $\begin{array}{l}\text { All require full-time } \\
\text { respiratory support }\end{array}$ \\
\hline $\begin{array}{l}\text { Facioscapulohumeral } \\
\text { muscular dystrophy }\end{array}$ & When onset $<20$ years & With infantile onset & With infantile onset & Slow & $\begin{array}{l}\text { Severe infantile onset type } \\
\text { is frequently associated with } \\
\text { sensorineural deafness }\end{array}$ \\
\hline \multicolumn{6}{|c|}{ Congenital muscular dystrophy } \\
\hline All types & Any age depending on severity & $\begin{array}{l}\text { Any age depending } \\
\text { on severity }\end{array}$ & $\begin{array}{l}\text { Any age depending } \\
\text { on severity }\end{array}$ & Slow & \\
\hline \multicolumn{6}{|l|}{ Congenital myopathy } \\
\hline Central core & $\begin{array}{l}\text { Uncommon except in severe } \\
\text { recessive type }\end{array}$ & Uncommon & Uncommon & Slow & $\begin{array}{l}\text { Susceptible to malignant } \\
\text { hyperthermia }\end{array}$ \\
\hline Minicore & Early while ambulation preserved & & & & \\
\hline Nemaline & $\begin{array}{l}\text { Early in severe neonatal form, } \\
\text { mild later onset form may develop } \\
\text { early while ambulation preserved }\end{array}$ & In severe form & In severe form & Slow & \\
\hline Myotubular & $85 \%$ in severe X-linked form & In severe form & In severe form & Slow & $\begin{array}{l}\text { Ophthalmoplegia, } \\
\text { rare coagulopathy } \\
\text { and liver haemorrhage }\end{array}$ \\
\hline Fibre type disproportion & Depends on genotype & Uncommon & Uncommon & & \\
\hline Charcot-Marie-Tooth & $\begin{array}{l}\text { With severe early onset, } \\
\text { especially with GDAP1 mutation }\end{array}$ & $\begin{array}{l}\text { With severe } \\
\text { early onset }\end{array}$ & $\begin{array}{l}\text { With severe } \\
\text { early onset }\end{array}$ & & $\begin{array}{l}\text { Stridor, especially with } \\
\text { GDAP1 mutation }\end{array}$ \\
\hline Pompe & $\begin{array}{l}\text { Infantile onset, may be early in } \\
\text { later onset while ambulation } \\
\text { preserved }\end{array}$ & Infantile onset & Infantile onset & $\begin{array}{l}\text { Infantile rapid, } \\
\text { late onset slow }\end{array}$ & $\begin{array}{l}\text { Variable relationship between } \\
\text { motor and respiratory progression }\end{array}$ \\
\hline
\end{tabular}

DMD, Duchenne muscular dystrophy; SMA, spinal muscular atrophy.

mass $^{16} 17$ and the high incidence of scoliosis and skeletal contractures. Immobility is associated with an increased risk of osteoporosis, which may worsen with steroid therapy for DMD.

In spite of several studies investigating body composition and energy requirements in patients with neuromuscular disorders, most notably in $\mathrm{DMD}$, there are few studies that specifically address the impact of nutrition on lung function in neuromuscular disorders. In a 10-year study of 162 boys with DMD, McDonald and colleagues ${ }^{18}$ found that the presence of obesity was unrelated to the decline in muscle strength, functional ability or lung function. In a small observational study of 10 patients with $\operatorname{DMD}(\mathrm{n}=6)$ and congenital muscular dystrophy $(n=4)$, Goldstein and colleagues ${ }^{19}$ looked at the effect of nightly over-feeding and whether it would reverse or improve muscle damage and consequently lung function, measured by vital capacity. These patients received nightly additional feeds with monitoring over a 3-month period. In the congenital muscular dystrophy group body weight did not change. In the DMD group, body weight increased. There was no change in lung function or measurable effects on daily activities in either group and there was diminished appetite during the day.

\section{Impact of scoliosis}

Scoliosis is a common feature of many neuromuscular conditions, with an incidence that generally reflects the severity of the associated weakness. Between $70 \%$ and $90 \%$ of boys with $\mathrm{DMD}^{20} 21$ and all children with SMA types 1 and 2 will develop clinically significant scoliosis, ${ }^{22}$ whereas it is less common in fascioscapulohumeral disease $(\sim 30 \%)^{23}$ and rare in Becker's muscular dystrophy (BMD). ${ }^{24}$

Scoliosis in the absence of NMW can affect lung function in a number of ways. Lateral curvature of the spine directly displaces the thoracic cage and diaphragm, limiting vital capacity by causing asymmetric inspiration and decreased chest 
wall compliance. The extent to which lung volume is reduced is variable; lung hypoplasia may occur in children with very early scoliosis, but in older children the lungs are likely to have developed normally, and compromise is due to mechanical limitations. Physiological dead space is increased, with a decreased tidal volume to dead space ratio, increasing the risk of carbon dioxide retention.

In children with NMW it can be difficult to separate the effects of weakness from those of scoliosis, since weaker children are also more likely to develop scoliosis. Inal-Ince and colleagues $^{25}$ studied 39 children and young adults with a range of neuromuscular disease, 22 with and 17 without scoliosis. Children with scoliosis had lower vital capacity than those without, and although these children were also older than those without scoliosis (13.6 vs 9.6 years), measures of respiratory muscle strength between the two groups were similar, suggesting that the difference in vital capacity was due to the presence of scoliosis rather than more severe weakness. A retrospective review of 43 children with SMA found that severity of scoliosis (Cobb angle) was inversely correlated with predicted vital capacity. ${ }^{26}$ In a longitudinal study of 25 boys with DMD published in 1983, Kurz and colleagues ${ }^{3}$ found that absolute vital capacity peaked at approximately 12 years of age, which in the pre-steroid era was around the time of loss of ambulation. Thereafter the vital capacity reduced in a linear fashion until the age of 17 when it appeared to remain stable at around 1 litre. The per cent predicted vital capacity (using armspan as a measure of standing height) fell in a linear fashion from age 7 years $(90 \%$ predicted) to age 20 years $(35 \%$ predicted). Age and the angle of thoracic scoliosis (but not lumbar scoliosis) predicted vital capacity, with each $10^{\circ}$ of thoracic scoliosis being equivalent to loss of $4 \%$ of predicted vital capacity (the same as that lost per year between 7 and 20 years of age). McDonald and colleagues reported a retrospective review of 162 boys with $\mathrm{DMD}$ followed at a single centre over a 10 -year period. ${ }^{18}$ The incidence of scoliosis increased steeply after loss of ambulation, with $50 \%$ of boys acquiring scoliosis between 12 and 15 years of age. Eighty subjects had lung function data, and similar to the data of Kurz and colleagues, there was a linear loss of vital capacity from around 10 to 19 years of age, followed by a plateau at around $25 \%$ predicted (a loss of approximately $7.5 \%$ per year). Scoliosis did not appear to influence the vital capacity, although the statistical approaches used were different to that of Kurz, and all scoliosis rather than just thoracic scoliosis was included in the analysis. Those with scoliosis had a higher rate of undefined pulmonary complications (39\% vs $14 \%$ ), suggesting a possible effect of scoliosis on cough effectiveness. Yamashita and colleagues ${ }^{27}$ reported a retrospective review of 36 boys with $\mathrm{DMD}, 32$ of whom had scoliosis, and found that boys with more severe and progressive scoliosis had lower peak vital capacity.

Progression of scoliosis during teenage years results from a combination of accelerated growth during adolescence, increasing weakness, and increased time spent in the sitting position. Galasko and colleagues ${ }^{28}$ showed that a regimen which encourages at least $2 \mathrm{~h}$ standing per day, using a combination of light weight orthotic aids and standing frames, can slow the progression of scoliosis and preserve lung function in boys with DMD.

\section{Sleep-disordered breathing and sleep-related hypoventilation}

Sleep in healthy individuals is associated with decreased ventilatory drive and reduced tone in the upper airway and intercostal muscles, especially during rapid eye movement (REM) sleep. In children with NMW these changes can result in sleep-related hypoventilation and/or obstructive sleep apnoea (OSA) (see table 4). Sleep-related hypoventilation is associated with hypoxaemia and hypercapnia, which can occur episodically during episodes of REM sleep with recovery during non-REM sleep, or in children with more severe weakness, throughout the period of sleep, often with a progressive hypercapnia. Hypoxaemia and hypercapnia can stimulate arousals and interfere with sleep quality.

Sleep-related hypoventilation may be exacerbated by coexisting OSA, resulting from weakness of pharyngeal muscles and adenotonsillar hypertrophy. Children who are obese or have associated macroglossia or craniofacial abnormalities are more likely to have coexisting OSA. Symptoms of snoring, parental reports of obstructed breathing, hyponasal speech, mouth breathing, nasal obstruction and adenotonsillar hypertrophy may suggest OSA, although clinical diagnosis correlates poorly with polysomnography (PSG). ${ }^{30}{ }^{31} \mathrm{~A}$ retrospective review of PSG studies of 32 boys with DMD showed that 10 (median age of 8 years) had OSA without significant hypoventilation. ${ }^{32}$ In older boys with $\mathrm{DMD}$, obstruction can be mixed with hypoventilation. ${ }^{33}$ Adenotonsillectomy can lead to resolution of symptoms related to obstruction. ${ }^{32}$

Myotonic dystrophy type 1 may be associated with daytime sleepiness in children and adults. In one study of 22 children with myotonic dystrophy, $76 \%$ reported fatigue, but only one had objective evidence of daytime sleepiness. There was a high prevalence of sleep fragmentation, associated with mixed apnoea and periodic limb movement. ${ }^{34}$ In adults myotonic dystrophy type 1 can be associated with short sleep latency, sleep-onset REM and low levels of hypocretin-1 in the cerebrospinal fluid, similar to that seen in narcolepsy, suggesting a possible primary dysfunction in the hypothalamic hypocretin system. ${ }^{35}$

\section{Daytime respiratory failure}

Daytime respiratory failure, characterised by daytime hypercapnia, tends to develop slowly as a result of progressive ventilation decompensation. It is usually preceded by sleep-related hypoventilation, and by recurrent episodes of respiratory exacerbation associated with respiratory infection or aspiration. Left untreated, daytime hypercapnia carries a poor prognosis. ${ }^{36}$ The development of daytime hypercapnia is usually an indication of progression of weakness, but may also reflect changes in chest wall or lung compliance as discussed above. Respiratory drive is usually normal, ${ }^{37} 38$ but there may be reduced ventilatory response to carbon dioxide in children who have prolonged periods of hypercapnia during sleep. In these children, nocturnal ventilation can reverse daytime hypercapnia.

Table 4 Sleep disorders associated with common neuromuscular diseases (adapted from Dhand and Dhand 2006) ${ }^{29}$

\begin{tabular}{ll}
\hline Disorder & Sleep abnormality \\
\hline Duchenne muscular dystrophy & $\begin{array}{l}\text { Obstructive sleep apnoea (younger patients) } \\
\text { Hypoventilation (older patients) }\end{array}$ \\
Spinal muscular atrophy & $\begin{array}{l}\text { Hypoventilation } \\
\text { Apnoea/hypopnea } \\
\text { Hypoventilation }\end{array}$ \\
Myotonic dystrophy & Apnoea /hypopnea \\
& Periodic limb movements \\
& Excessive daytime sleepiness \\
Peripheral neuropathies (eg, & Hypoventilation \\
Charcot-Marie-Tooth disease) & Frequent arousals \\
\hline
\end{tabular}




\section{Acute respiratory failure}

In most children with NMW, episodes of acute respiratory failure are associated with respiratory tract infections and retention of airway secretions as discussed above. Respiratory load is increased in these situations and worsened further by ineffective coughing and mucus retention. ${ }^{39}$ In children with congenital myasthenic syndromes, weakness can be variable and unpredictable. Acute respiratory failure and/or sudden apnoea can occur due to acute severe decrease in muscle strength, often but not necessarily triggered by viral respiratory illnesses. ${ }^{40}$ Box 1 lists the potential precipitating factors of acute respiratory failure in children with neuromuscular disease.

\section{IDENTIFYING CHILDREN AT RISK OF RESPIRATORY COMPLICATIONS \\ Clinical assessment}

Clinical assessment of respiratory health should be part of every medical consultation for children with NMW. The purpose and nature of all assessments should be explained to children and their carers. An assessment should be made of the extent, pattern and progression of muscle weakness, the degree of ambulation and the degree of muscle fatigability. Any change in the strength of voice or cough should be documented, and problems with drooling, difficulties in clearing secretions, choking with foods, or difficulties with chewing identified. Posture, seating and the development of kyphosis and scoliosis should be regularly assessed as should growth and nutritional status. An increase in frequency and severity of respiratory infections is likely to occur with deteriorating muscle strength and careful attention should be given to establishing how these have been managed and whether they have required hospital admission for intensive treatment.

Sleep-disordered breathing in patients with neuromuscular disease may be caused by sleep-related hypoventilation or by OSA or a combination of both. The symptoms of nocturnal hypoventilation include disturbed sleep, morning headache, morning anorexia or nausea, daytime sleepiness, fatigue and poor concentration. These symptoms are non-specific and do not reliably predict the presence of nocturnal hypoventilation. Symptoms often develop gradually over several weeks or months and may not be recognised by children or their parents. Symptoms relating to OSA may include snoring, and variable patterns of snoring, breathing effort and arousal. When weakness is severe, daytime hypoventilation may occur. The symptoms of daytime hypoventilation are headache, nausea,

Box 1 Precipitating factors of acute respiratory failure in children with neuromuscular disease (adapted from Racca et $\left.a\right|^{41}$ )

Upper respiratory tract infections

Pneumonia

Atelectasis

Cardiac failure secondary to cardiomyopathy and/or arrhythmia

Sedative drugs

Aspiration

Pneumothorax

Pulmonary embolism

Acute gastric distension associated with use of non-invasive ventilation dyspnoea, tachycardia, sweating, peripheral vasoconstriction or vasodilation, fatigue and anxiety. Once again these are nonspecific.

\section{Evidence}

Clinical predictors for risk of respiratory complications

There are no studies which have formally evaluated clinical assessment in isolation as a means of identifying the risk of respiratory complications in patients with neuromuscular disease. Evidence addressing whether clinical assessment can predict sleep-disordered breathing comes from three studies. ${ }^{32} 4243$

In a cross-sectional study, two measures of global function (Barthel Index and Egen Klassification) correlated significantly with spirometry, in particular vital capacity, when applied to 26 patients with $\mathrm{DMD}$ (mean age 12.7 years, range $7-22$ years). ${ }^{42}$ In a descriptive retrospective study of 34 patients with DMD aged $1-15$ years (median 10 years) who attended a sleep clinic over a 5-year period, ${ }^{32}$ symptoms and lung function were correlated with contemporary sleep study data. There was no association between the diagnosis of sleep-disordered breathing on sleep study, and the presence or absence of symptoms. In a study of 46 children aged 6-17 years with a heterogeneous group of muscle diseases, clinical measures were correlated with sleep study findings. Scoliosis on clinical assessment predicted nocturnal hypoventilation with a sensitivity of $85 \%$ and a specificity of $73 \%{ }^{43}$

\section{Clinical predictors of survival}

The evidence that clinical assessment can predict survival in patients with neuromuscular disease comes from two studies from the same group, both of which relate to patients with DMD. ${ }^{44} 45$ The first study is a retrospective longitudinal descriptive study of 18 patients with DMD (age $>14$ years, median 17.8 years). ${ }^{45}$ Sixteen patients died during the course of the study. Age at non-ambulation and body mass index did not predict survival duration or age at death in these patients. In a larger retrospective longitudinal study, the same group looked at 58 non-ventilated patients with $\mathrm{DMD}$ age $>10$ years over a period of 14 years. ${ }^{44}$ Thirty-seven patients died during the study. This study confirmed that age at non-ambulation and body mass index did not predict age at death. Maximal height derived from armspan did predict survival.

\section{Evidence statements}

Clinical assessment in isolation is a poor predictor of sleepdisordered breathing in children with DMD (evidence level 3).

Clinical assessment in isolation is a poor predictor of survival in children with DMD (evidence level 3).

\section{Recommendation}

- Clinical assessment of respiratory health should be part of every medical consultation for children with NMW and should be directed towards identifying progressive muscle weakness, ability to cope with respiratory infection, aspiration, progression of scoliosis and sleep-disordered breathing. [D]

\section{Spirometry}

In healthy children repeatable spirometry using visual incentives is generally achievable from 6 years of age. Children who are weak may be unable to achieve flow limitation while performing spirometry even if they have good technique, and forced manoeuvres may not be possible. Vital capacity, which is determined by inspiratory muscle strength and lung and chest wall compliance, can be reliably measured in almost all weak subjects by using a slow manoeuvre whereby the child is asked to breathe in as deeply as possible and then to breathe out for as long as possible through the spirometer. 
Height and age are normally used to predict expected lung function values in children. In children with neuromuscular disease and scoliosis, height measurement may be unreliable. Other body measurements that do not require the patient to stand and which are not confounded by body deformity can be used to accurately predict height and lung function. Most centres in the UK use armspan or ulna length as a surrogate measure for height. Ulna length may be easier to measure in patients with significant deformity or contractures.

Vital capacity is usually measured in the standing or sitting position to allow gravity to assist with diaphragmatic descent. When measured in the supine position vital capacity is lower, particularly in subjects with diaphragmatic weakness, and it has been suggested that supine vital capacity may be useful in monitoring disease progression. ${ }^{46}$ Subjects with NMW may have difficulty in creating a tight seal with their lips around a conventional mouthpiece. This problem can be addressed by using a flanged mouthpiece or by using a face mask held firmly over the mouth and nose as the spirometry interface. Vital capacity has been shown to be a valuable predictor of susceptibility to infection and the need for respiratory support in children with muscle disease. Vital capacity correlates with survival in children and adolescents with DMD. Vital capacity is less useful in patients with rigid spine syndromes who may have severe nocturnal hypoventilation despite preserved vital capacity, peripheral muscle strength and ambulation.

\section{Evidence}

\section{Armspan as a predictor of height in lung function assessment}

The evidence for using armspan to predict height in children comes from one large longitudinal study in healthy individuals ${ }^{47}$ and two smaller studies of patients with scoliosis. ${ }^{48} 49$ Three smaller case-control cross-sectional field studies, all from the same centre but performed in Nigeria, the Philippines and India, provide data on applying armspan predictions of height in disabled children. ${ }^{50-52}$

In the first study 512 healthy children were followed with annual measurements for armspan and height for 6 years, giving rise to a total of 2367 measurements. ${ }^{47}$ Armspan to height ratio was extremely close to 1.0 across all ages. The two scoliosis studies recruited children and adults and also found good correlation between armspan and height, but noted that sex and age affected armspan to height ratio. ${ }^{48} 49$

In the three case-control field studies, height and armspan measurements were taken from normal controls and disabled children, all aged $<10$ years. Regression equations were derived using the control children and tested using the data from the disabled children. Taken together, 325 normal controls were measured. Strong correlations between armspan and measured height were identified in all three studies. Predicted heights derived using armspan were not significantly different to actual height measured in the disabled children. ${ }^{50-52}$

\section{Ulna length as a predictor of height in lung function assessment}

The evidence for using ulna length to predict height in normal children comes from one large cross-sectional study in which 2343 normal children and adolescents aged 5-19 years were studied. Height, arm span, tibial length and ulnar length were all measured. Regression equations were found to be best for ulnar length. Ulnar length was the only parameter that was not significantly different between ethnic groups included in the study. ${ }^{53}$ A parallel study by the same group on the same cohort produced regression equations incorporating age and ulna length to predict expected lung function $\left(\mathrm{FEV}_{1}\right.$ and FVC) directly. ${ }^{54}$
Spirometry with visual incentives can improve lung function measures in children with DMD

Evidence for visual incentive spirometry (IS) comes from one randomised crossover intervention study in 47 children with DMD aged $6-19$ years (mean age 12.6 years). ${ }^{55}$ The boys were categorised as having moderate, mild or no cognitive impairment. In those with moderate intellectual or behavioural impairment there was a significant improvement in lung function when computerised visual incentives were used.

\section{Vital capacity as a predictor}

The evidence that vital capacity can predict susceptibility to infection comes from one retrospective questionnaire study that looked at 46 patients aged 8-16 years with a mixed group of muscle diseases. ${ }^{56}$ Many parameters were studied, including more specific tests of respiratory muscle strength and cough peak flow (CPF). Vital capacity had the strongest correlation with the number of chest infections and days of antibiotic treatment in the preceding year. A vital capacity of $<1.11$ litres predicted risk of chest infection with a sensitivity of $90.5 \%$ and a specificity of $70.8 \%$.

The evidence that vital capacity can predict sleep-disordered breathing in patients with neuromuscular disease comes from five cross-sectional studies. In two German studies, the first in 61 children aged 5-18 years with a heterogeneous group of muscle diseases, and the second in 42 adults aged 14-63 years also with a heterogeneous group of muscle diseases, inspiratory vital capacity (IVC) of $<60 \%$ expected predicted sleep-disordered breathing and IVC $<40 \%$ expected predicted nocturnal hypoventilation. ${ }^{57}$ Another cross-sectional study looked at 114 patients with $\mathrm{DMD}$ aged $16-23$ years. In this cohort, a vital capacity $<1820 \mathrm{ml}$ predicted nocturnal hypoventilation, although the sensitivity and specificity for this cut-off value are not given. ${ }^{59}$ In a study of 19 patients with $\mathrm{DMD}$ age over 12 years (mean 18.6 years), $\mathrm{FEV}_{1}$ and $\mathrm{FVC}$ predicted the total sleep time with oxygen saturations below $90 \% .{ }^{60}$ In a study of 46 children aged 6-17 years with a heterogeneous group of muscle diseases, clinical measures were correlated with PSG; vital capacity of $<70 \%$ predicted indicated the presence of nocturnal hypoventilation with a specificity of $71 \%$ and sensitivity of $64 \%{ }^{43}$

The evidence that vital capacity can predict daytime respiratory failure (hypercapnia) in patients with neuromuscular disease comes from three of the cross-sectional studies described above $e^{57-59}$ and a further cross-sectional study in a cohort of children and young adults with $\mathrm{DMD} .^{61}$ In the two German studies, ${ }^{57} 58$ vital capacity $<25 \%$ predicted diurnal respiratory failure (daytime hypercapnia). In the cohort of 114 patients with DMD aged 16-23 years, ${ }^{59}$ vital capacity $<680 \mathrm{ml}$ predicted daytime hypercapnia. In this study vital capacity was the best predictor of daytime hypercapnia compared with other lung function parameters, including tidal volume, respiratory rate and maximal inspiratory pressure (MIP). In the fourth study of 52 patients with DMD aged $7-25$ years, vital capacity was again shown to be highly correlated with daytime partial pressure of carbon dioxide $\left(\mathrm{PaCO}_{2}\right){ }^{61}$

The evidence that vital capacity can predict survival in patients with neuromuscular disease comes from two retrospective longitudinal descriptive studies from the same group, both of which relate to children with DMD. ${ }^{44} 45$ The first study included 18 patients with $\mathrm{DMD}$ and median age 17.8 years followed over a 10 -year period. ${ }^{45}$ The second study included 58 non-ventilated patients with DMD aged over 10 years followed over a period of 14 years. ${ }^{44}$ In these studies, maximum vital 
capacity, rate of decline in vital capacity and current vital capacity all correlated with survival in non-ventilated patients. In the first study, age at which vital capacity fell below 1 litre was associated with age at death. ${ }^{45}$ In the larger second study, when the vital capacity fell below 1 litre the median survival was 3.1 years and the 5 -year survival was $8 \%{ }^{44}$

\section{Tests of respiratory muscle strength}

A number of tests have been developed that have the potential to provide information about specific weakness in inspiratory, expiratory and bulbar muscle groups. These muscle groups contribute differently to ventilation, secretion clearance and airway protection, and are affected to a varying degree in different neuromuscular diseases. Several studies have evaluated these measures as potential predictors of respiratory complications in patients with neuromuscular disease. The majority of studies show no benefit over vital capacity.

MIP is a measure of inspiratory muscle strength. It is measured using a manometer connected to a mouthpiece. The patient is asked to breathe out to residual volume and then asked to take a maximal inspiratory effort against an occluded airway. There is a small leak in the system to avoid pressure being generated by cheek muscles. The maximum negative pressure generated after a period of $1 \mathrm{~s}$ is recorded as the MIP.

Sniff nasal inspiratory pressure (SNIP) is another measure of inspiratory muscle strength. It measures ballistic rather than sustained inspiratory strength. SNIP is measured using a tightly fitting plug placed inside one nostril. A catheter connects the plug to a pressure transducer. The sniff is performed through the contralateral patent nostril. SNIP is easier to perform than MIP.

Maximal expiratory pressure (MEP) is a measure of expiratory muscle strength using a manometer connected to a mouthpiece. The patient is asked to breathe in to total lung capacity and then asked to take a maximal expiratory effort against an occluded airway. The cheeks are supported by the patient's or assistant's hands. There is a small leak in the system to avoid pressure being generated by cheek muscles. The maximum pressure generated after a period of $1 \mathrm{~s}$ is recorded as the MEP.

\section{Evidence}

\section{Maximum inspiratory pressure}

Evidence that MIP can predict respiratory complications comes from five studies. MIP has been shown to be highly correlated with vital capacity in four cross-sectional studies of children and young adults with muscle disease, all of which have been mentioned in the section on vital capacity. ${ }^{57-59} 61$ The close relationship between MIP and vital capacity means that MIP is also capable of predicting sleep-disordered breathing, nocturnal hypoventilation and daytime hypercapnia in patients with muscle weakness. In the German cross-sectional study of 61 children aged 5-18 years with a heterogeneous group of muscle diseases, ${ }^{58} \mathrm{MIP}<4.0 \mathrm{kPa}$ was associated with sleep-disordered breathing and MIP $<2.5 \mathrm{kPa}$ with nocturnal hypoventilation, but both outcomes were better predicted by vital capacity. Threshold values for MIP were different in the German adult study. ${ }^{57}$ In the retrospective questionnaire study that looked at 46 patients aged 8-16years with a mixed group of muscle diseases, MIP unlike vital capacity did not correlate with the number of infections and days of antibiotic treatment in the preceding year. ${ }^{56}$ Finally, in a small study of patients with $\mathrm{DMD}$, MIP did not predict total sleep time with oxygen saturations below $90 \%$ when spirometry and daytime $\mathrm{PaCO}_{2}$ were predictive. $^{60}$

\section{Sniff nasal inspiratory pressure}

Evidence that SNIP may be useful in the assessment of respiratory complications in neuromuscular disease comes from studies that show correlation between SNIP and MIP and with vital capacity. In a study of 116 normal children aged 4-11 years, SNIP was slightly greater than MIP but was highly correlated with MIP. ${ }^{62}$ In a study of 126 children and adults with neuromuscular disease or skeletal disorders, SNIP was easier to perform than MIP in the patients with neuromuscular disease and correlated well with MIP. ${ }^{63}$ SNIP correlated with FVC in a study of 41 children with neuromuscular disease; in this study reliable SNIP measurements were obtained in all children, whereas FVC measurements could only be obtained in 25 of the 41 children, suggesting that children may find SNIP manoeuvres easier to perform than FVC. ${ }^{64}$ SNIP performance, and therefore reliability as a measure, improves with repeated attempts. ${ }^{65}$

\section{Maximal expiratory pressure}

Evidence that MEP may be useful in the assessment of respiratory complications in neuromuscular disease comes from two studies. In a cross-sectional study of 52 patients with DMD aged $7-25$ years $^{61}$ MEP correlated with vital capacity. This study suggested that in this patient group MEP may decline before MIP and vital capacity as muscle weakness progresses. In the retrospective questionnaire study that looked at 46 patients aged 8-16 years with a mixed group of muscle diseases, MEP correlated with frequency of infections and days of antibiotics in the preceding year, but the correlation was less good than with vital capacity or $\mathrm{CPF}^{56}$

\section{Evidence statements}

Ulna length or armspan can be used instead of height to accurately predict expected lung function values (evidence level $2++)$

Vital capacity is a valuable predictor of susceptibility to infection, need for respiratory support and survival in children and adolescents with DMD (evidence level $2+$ ).

Tests of respiratory muscle function such as maximum inspiratory pressure, SNIP or MEP provide little or no additional information when compared to vital capacity (evidence level 2-).

\section{Recommendations}

- Ulna length or armspan should be used to predict lung function in children with neuromuscular disease whose height cannot be accurately measured. [B]

- Vital capacity should be measured in all patients with neuromuscular disease who are capable of performing spirometry as part of the respiratory assessment. [C]

\section{Cough peak flow}

The ability to cough requires coordinated use of inspiratory, bulbar and expiratory muscles. Cough effectiveness can be assessed by CPF. CPF can be measured using a face mask or mouthpiece connected to a peak flow meter into which the child makes a maximal cough effort after deep inspiration. Healthy adults have $\mathrm{CPF} \geq 400$ litres/min. CPF measured during an augmented cough (see section on airway clearance) is called 'assisted CPF'. Adults, and probably children over 12 years of age, who have a $\mathrm{CPF}<270$ litres/min are vulnerable to respiratory failure during otherwise trivial viral respiratory tract infections as a result of secretion retention. Adults and probably children over 12 years of age with $\mathrm{CPF}<160$ litres/min are not able to clear their airway secretions effectively and in situations where an artificial airway is in place, successful extubation or decannulation can be difficult and is likely to fail without 
frequent use of augmented cough techniques and immediate use of NIV.

\section{Evidence}

A study of 49 adults requiring ventilation via endotracheal or tracheostomy tubes with a range of neuromuscular problems found that patients with an assisted $\mathrm{CPF}<160$ litres/min could not be successfully extubated or decannulated, ${ }^{66}$ suggesting that a $\mathrm{CPF} \geq 160$ litres/min is required to effectively clear secretions and maintain airway patency. A subsequent study of 157 invasively ventilated adults and an unspecified number of children, including 139 patients intubated for acute respiratory failure, reported a $100 \%$ success rate for extubation for patients with $\mathrm{CPF} \geq 160$ litres $/ \mathrm{min}^{67}$ and an $80 \%$ success rate for patients with $\mathrm{CPF}<160$ litres/min. The reasons suggested for the improved success rate in patients with very low CPF were greater staff and patient experience of mechanical cough assist devices and NIV and a requirement for the patient's oxygen saturation levels to be $95 \%$ or greater for $12 \mathrm{~h}$ prior to an extubation attempt, rather than the previous level of $92 \%$. Three studies in adults with NMW have shown that an assisted or unassisted CPF over 270 litres/min is sufficient to protect against acute respiratory deterioration associated with respiratory infection. ${ }^{39} 6869$ The likely reason for the higher CPF required to protect against respiratory deterioration is the fall in respiratory muscle strength that is known to occur in patients with NMW during acute infection. ${ }^{70}$ This means that a CPF $<270$ litres/min when the patient is well may fall below the critical level of 160 litres/min during an infective exacerbation, leading to secretion retention and respiratory failure. In a study of children and adults with $\mathrm{DMD}$, a vital capacity of $<2.1$ litres best predicted a $\mathrm{CPF}<270$ litres/min. ${ }^{71}$

Care should be taken when applying CPF thresholds derived from the adult population to children. Standard values for CPF for normal children aged 4-18 years of age have been published. ${ }^{72}$ The 5 th centile CPF ranged from 110 litres/min in 4-year old girls to 518 litres/min in 18-year old boys. In a study of 46 children and young adults with NMW aged from 4 to 20 years (mean age $12.7 \pm 3.7$ years) $\quad \mathrm{CPF}<160$ litres $/ \mathrm{min}$ predicted frequency of severe chest exacerbation sensitivity of $75 \%$ and specificity of $79 \%{ }^{56}$ This study used absolute CPF values rather than those corrected for age and height. The value of 160 litres/min is significantly lower than that identified in adults as protective against recurrent chest infections (270 litres/ $\mathrm{min})$. There is currently insufficient evidence in children to provide thresholds of absolute CPF values that will reliably predict risk of acute respiratory deterioration. It may be reasonable to apply adult thresholds to older children (those over 12 years of age), particularly those with DMD when vital capacity usually peaks at around 12 years of age.

\section{Evidence statement}

In adults with NMW, a CPF of 160 litres/min is necessary for effective secretion clearance and a CPF of 270 litres/min or more is associated with resilience to respiratory infection. Equivalent age-adjusted values for children are not available (evidence level 3).

\section{Good practice point}

- CPF should be used as part of the assessment of effective secretion clearance in children with neuromuscular disease over the age of 12 years. [ $\mathrm{V}]$

\section{Measurement of daytime blood gases}

In general blood gas analysis in children is achieved using arterialised capillary samples. Results may be confounded by sampling from poorly perfused tissues or by procedure-related hyperventilation. Daytime hypercapnia indicates hypoventilation during wakefulness; children with daytime hypercapnia will also have nocturnal hypoventilation. A daytime capillary blood gas sample showing a raised plasma bicarbonate level may indicate the presence of nocturnal hypercapnia.

\section{Evidence}

The evidence that daytime blood gases can identify patients with nocturnal hypoventilation comes from two cross-sectional studies. In a small study of 19 patients with $\mathrm{DMD}$ age over 12 years (mean 18.6 years), daytime $\mathrm{PaCO}_{2}$ correlated strongly with total sleep time with oxygen saturations below $90 \%$. A daytime $\mathrm{PaCO}_{2}>45 \mathrm{~mm} \mathrm{Hg}$ predicted nocturnal hypoventilation (arbitrarily defined as oxygen saturation below $90 \%$ for $>2 \%$ of total sleep time) with a $91 \%$ sensitivity and $75 \%$ specificity. ${ }^{60}$ In a study of 61 children aged $5-18$ years with a heterogeneous group of muscle diseases, a daytime $\mathrm{PaCO}_{2}>40 \mathrm{~mm} \mathrm{Hg}$ predicted nocturnal hypoventilation with a sensitivity of $92 \%$ and specificity of $72 \%{ }^{58}$

Evidence that daytime $\mathrm{PaCO}_{2}$ can predict survival comes from one study. In a retrospective longitudinal descriptive study of 18 patients with DMD (age over 14 years, median 17.8 years), daytime $\mathrm{PaCO}_{2}$ correlated with vital capacity and with survival. In stepwise multiple regression analysis for survival, $\mathrm{PaCO}_{2}$ provided no further predictive value in addition to vital capacity. ${ }^{45}$

\section{Overnight sleep monitoring}

Decline in respiratory function in patients with progressive neuromuscular disease eventually leads to respiratory failure and diurnal hypoventilation. Respiratory mechanics are compromised in sleep, and hypoventilation in patients with NMW therefore first becomes apparent during sleep. Sleep staging in children can be broadly categorised into active (REM) sleep and quiet (non-REM) sleep. Diaphragmatic strength is preserved in all sleep stages. Intercostal, upper airway and accessory muscle function is reduced in active sleep because of muscle atonia. Nocturnal hypoventilation in progressive NMW therefore occurs first in active sleep when muscle strength is most compromised. As respiratory weakness increases, hypoventilation progresses to all sleep stages, before progressing to daytime hypoventilation. Overnight sleep monitoring is used to identify patients who have nocturnal hypoventilation.

There are a number of approaches to overnight monitoring that can be used to gain information about sleep-disordered breathing. These approaches are discussed in order of increasing complexity.

\section{Pulse oximetry}

The simplest assessment of sleep-disordered breathing is nocturnal oxygen saturation monitoring. Oxygen saturation traces may be difficult to interpret as apparent desaturation can be a consequence of movement artefact and poor signal. Technically satisfactory oximetry requires a correctly placed probe, typically sampling $>60$ times/s, with an output averaged over $4-10 \mathrm{~s}$ and displayed at $1 \mathrm{~s}$ intervals. The data need to be displayed with the plethysmographic waveform to allow the quality of the data to be assessed, and in a format which allows several hours of oximetry to be seen on a single screen and interrogated as necessary.

True oxygen desaturation may be a consequence of OSA or hypoventilation. OSA and hypoventilation occur more frequently in active sleep. If OSA is present, oximetry traces 
may show a typical saw tooth pattern of repeated cyclical oxygen desaturation in active sleep, whereas there may be periods of sustained oxygen desaturation if hypoventilation is occurring. These patterns are not always present such that OSA and hypoventilation cannot be reliably distinguished from each other using oximetry. Mild OSA may be present even if an oxygen saturation trace is normal, ${ }^{73}$ and hypercapnia has been reported in children using NIV or continuous positive airway pressure (CPAP) despite normal oximetry (oxygen saturation never $<92 \%) .{ }^{74}$ Despite these observations, the Guideline Committee took the view that clinically significant hypoventilation as a consequence of muscle weakness is unlikely to occur in the absence of desaturation below 93\%, and that technically satisfactory oximetry alone is an acceptable method of screening for hypoventilation in asymptomatic children with NMW when carbon dioxide monitoring is not readily available. The Committee would not recommend oximetry alone as a means of monitoring the effectiveness of ventilation in children using NIV, nor was it felt to be sufficient to exclude sleep-disordered breathing in symptomatic children. Oxygen saturation monitoring has been shown to be feasible at home in patients with DMD. ${ }^{75}$

\section{Oxycapnography}

Portable devices that can perform transcutaneous carbon dioxide measurement enable simple testing for oxygen and carbon dioxide monitoring during sleep. Periods of sustained oxygen desaturation coupled with sustained hypercapnia in active sleep strongly suggest hypoventilation, although this pattern is also possible in the context of severe OSA. Carbon dioxide measurements can be obtained using end-tidal or transcutaneous devices. Some transcutaneous devices allow simultaneous measurement of carbon dioxide and oxygen saturation and are suitable for subjects of all ages, including adults. In a retrospective review of 609 paediatric patients who had had PSG, transcutaneous carbon dioxide correlated well with end-tidal carbon dioxide measurements. ${ }^{76}$

\section{Respiratory polygraphy (cardiorespiratory sleep studies)}

The complexity of sleep polygraphy (cardiorespiratory sleep studies) varies between institutions. The core measurement channels include oximetry with plethysmograph signal, transcutaneous or end-tidal carbon dioxide monitoring, ECG, chest and abdominal inductance bands for assessment of thoracoabdominal breathing patterns, movement probe and nasal flow (detected either by nasal thermistor or pressure sensor). Other channels may include sleep position and video monitoring.

Sleep assessment in children may contain significant artefact, because of poor compliance with equipment and movement in sleep. The advantage of more complex studies is that more detailed information is collected, and artefact can be more easily excluded. The most important channel for identifying artefact is the oximetry plethysmograph which gives a measure of reliability to the oximetry value. Disadvantages of more complex studies include poor tolerance of intrusive equipment, which may interfere with the quality of the overall study. Nasal flow measurements can be particularly intrusive and may be counterproductive in some patients.

\section{Polysomnography}

PSG involves monitoring of sleep with continuous EEG, electromyogram and electrooculogram in addition to comprehensive monitoring of cardiorespiratory function (oximetry with plethysmograph signal, carbon dioxide monitoring, ECG, chest

and abdominal inductance bands, nasal flow, movement probe, sleep position and video monitoring). Full PSG provides information about sleep efficiency and sleep quality as well as detailed characterisation of respiratory patterns and abnormalities.

\section{Timing and frequency of overnight monitoring}

There is some evidence that vital capacity is a valuable predictor of nocturnal hypoventilation, as discussed above. The other indications for assessment of sleep-associated hypoventilation suggested in table 5 are based on the expert opinion of the Guideline Committee.

There are no studies that evaluate how frequently sleep studies should be performed. The American Thoracic Society Consensus Statement for DMD suggests annual assessment. ${ }^{77}$ In young children whose rate of disease progression is uncertain, or in older children who have shown a clinical deterioration or are suffering with repeated infections, sleep assessment may need to be more frequent than once a year.

Sleep data should be interpreted by an experienced reporter with appropriate training in sleep medicine. Most centres in the UK will have a dedicated sleep physiologist responsible for interpreting sleep studies.

\section{Evidence}

Evidence that overnight sleep monitoring can be used to predict progression in neuromuscular disease comes from two studies. In a randomised controlled trial of NIV versus no treatment in 26 patients with neuromuscular or chest wall disease who had nocturnal hypercapnia but daytime normocapnia, control patients progressed to daytime hypercapnia and/or progressive symptoms within 2 years. ${ }^{78}$ In a retrospective longitudinal descriptive study of 18 non-ventilated patients with DMD (age $>14$ years, median 17.8 years), the minimum $\mathrm{PaO}_{2}$ measured at night in a group of adolescents with DMD correlated with subsequent survival. ${ }^{45}$

\section{Pulse oximetry}

There are no studies that specifically evaluate whether oxygen saturation monitoring alone is adequate or necessary as a method for screening for nocturnal hypoventilation in children or adults with neuromuscular disease. There is one study of 50

Table 5 Indications for overnight sleep monitoring in children with neuromuscular weakness

\begin{tabular}{ll}
\hline Indication & Notes \\
\hline Vital capacity $<60 \%$ predicted & $\begin{array}{l}\text { Children generally need to be over } 6 \text { years } \\
\text { of age to produce reliable spirometry. } \\
\text { In boys with DMD, a vital capacity of over } \\
1.8 \text { litres indicates that nocturnal } \\
\text { hypoventilation is very unlikely to be } \\
\text { present }\end{array}$ \\
& Inability to walk is a measure of moderate \\
Loss of ambulation because of & to severe muscle weakness \\
progressive weakness, or children &
\end{tabular}

progressive weakness, or children who never attain the ability to walk Infants with weakness

Children with symptoms of obstructive sleep apnoea or hypoventilation

Children with diaphragmatic weakness

Children with rigid spine syndrome 
children (23 with neuromuscular disease) using NIV or CPAP for sleep-disordered breathing which found that oximetry alone failed to identify all those with hypercapnia (transcutaneous $\mathrm{CO}_{2}>50 \mathrm{~mm} \mathrm{Hg}$ for $10 \%$ of the recording time or for at least 5 continuous minutes). ${ }^{74}$

\section{Oxycapnography}

There are no studies that specifically evaluate whether transcutaneous carbon dioxide monitoring coupled with oxygen saturation monitoring is adequate or necessary as a method for screening for nocturnal hypoventilation in children or adults with neuromuscular disease. The information provided by carbon dioxide monitoring in addition to oximetry was explored in a small study of 11 patients aged $15-75$ years with a heterogeneous group of muscle diseases. Oximetry was compared with oxycapnography using end-tidal carbon dioxide. All five patients who had significant hypercapnia (end tidal $\mathrm{CO}_{2}>47 \mathrm{~mm} \mathrm{Hg}$ ) also had evidence of oxygen desaturation. ${ }^{79}$

\section{Respiratory polygraphy}

There are no studies that specifically evaluate whether respiratory polygraphy is adequate or necessary as a method for screening for nocturnal hypoventilation in children or adults with neuromuscular disease.

\section{Polysomnography}

There are no studies that specifically evaluate what level of sleep monitoring is necessary to identify children with neuromuscular disease who have sleep-disordered breathing. Two consensus statements for the standard of care in DMD and SMA respectively both endorse full PSG as the study of choice for children who are at risk of sleep-disordered breathing. ${ }^{77} 80$ These documents acknowledge the fact that detailed PSG or sleep polygraphy is not universally available and recommend the most detailed level of study possible given the available resources.

\section{Recommendation}

- Assessment for sleep-disordered breathing should be carried out no less than annually for children who have a vital capacity of $<60 \%$ predicted and for children who have become non-ambulant because of progressive muscle weakness or who never attain the ability to walk. [D]

\section{Good practice points}

- Assessment for sleep-disordered breathing should be carried out no less than annually for all infants with weakness, children with NMW who have symptoms of OSA or hypoventilation, children with clinically apparent diaphragmatic weakness and children with rigid spine syndromes. [ $\sqrt{ }]$

- In young children whose rate of disease progression is uncertain, or in older children who have shown a clinical deterioration or who are suffering with repeated infections, or who develop symptoms of sleep-disordered breathing, sleep assessment may need to be more frequent than once a year. $[\sqrt{ }]$

- Technically satisfactory overnight oximetry (see text) which shows that oxygen saturation is maintained at $93 \%$ or above is considered to be sufficient to exclude clinically significant nocturnal hypoventilation in asymptomatic children with NMW. $[\sqrt{ }]$

- All children with abnormal overnight oximetry should undergo more detailed sleep monitoring with at least oxycapnography. $[\sqrt{ }]$

- When there is doubt about the cause of sleep-disordered breathing, overnight PSG or sleep polygraphy should be performed. $[\sqrt{ }]$

- Portable overnight oxycapnography or polygraphy in the home may be the most appropriate option for some patients. [ $\sqrt{ }]$
- Interpretation of overnight sleep monitoring should be performed by an experienced reporter with appropriate training in sleep medicine. $[\sqrt{ }]$

\section{Studies to identify aspiration lung disease}

Feeding difficulties are well described in paediatric neuromuscular disease. Swallowing difficulties will increase the risk of aspiration lung disease. Symptoms suggestive of progressive difficulty with swallowing include coughing or choking with feeds, and increasing time taken over mealtimes. ${ }^{81}$ Aspiration may be silent and manifest as recurrent pneumonia. There is no gold standard investigation for identifying aspiration. If aspiration lung disease is suspected, most centres will involve a speech and language therapist to make an assessment of feeding and consider carrying out a videofluoroscopic swallow study (VFSS) if there are concerns regarding the safety of swallow. Feeding assessment will help formally identify whether oral manipulation of food, impairment of chewing or a compromised swallow are affecting the patient's ability to feed safely. The impact of positioning and head control is an important consideration.

If there are concerns regarding safety of swallowing after a swallow assessment has been made, then a VFSS should be considered. These studies provide detailed information about the oral and pharyngeal stages of the swallow manoeuvre. Frank laryngeal aspiration can be documented if it occurs. Penetration of the larynx with coating of the underside of the epiglottis is abnormal and can lead to aspiration, especially when patients become fatigued. Different muscle diseases will be associated with different swallowing problems. Patients with severe weakness such as children with SMA type 1 may have vocal cord paralysis and silent aspiration. ${ }^{80}$ In children with $\mathrm{DMD}$, the oral phase is most compromised and contributes to the risk of choking even when the pharyngeal phase is relatively preserved. ${ }^{82}$ Children with NMW may only aspirate when unwell, when they may be weaker and having to cope with increased airway secretions. VFSS captures a snapshot in time and can be falsely reassuring. Even if the procedure is repeated on several occasions, particular features of a child's swallow including aspiration events may be missed; it is therefore important that VFSS results are interpreted alongside the case history and clinical evaluation. In a study of 30 patients with DMD aged 6-31, who had repeated chest infections or difficulties with choking, reported swallowing problems were not always associated with aspiration on VFSS. ${ }^{82}$

Direct visualisation of swallowing can be achieved using a flexible endoscope. This involves placing a flexible endoscope via the nose into the posterior pharynx above the larynx to observe the larynx directly during the swallow manoeuvre. This requires skill and experience and is not currently a widely used approach for evaluating swallowing in children with neuromuscular disease.

\section{Evidence}

Four observational studies ${ }^{81-84}$ in a total of 57 children with $\mathrm{DMD}$, congenital myopathy or congenital muscular dystrophy using a combination of clinical assessment and VFSS have shown that VFSS is often abnormal in children with NMW, but that it does not always correlate with symptoms. In one study of 14 children with merosin-negative muscular dystrophy, five children who had either laryngeal penetration or tracheal aspiration shown on VFSS underwent gastrosotomy and stopped feeding orally, with subsequent weight gain a reduction of respiratory exacerbations. ${ }^{81}$ Of 24 boys and young men with $\mathrm{DMD}$ assessed because of a history of swallowing difficulties or 
choking and recurrent chest infections, there was no evidence of tracheal aspiration on VFSS. ${ }^{82} 84$

\section{Good practice point}

- Children with neuromuscular disease with a history of swallowing difficulties should have a feeding assessment by a speech and language therapist including a video fluoroscopy swallow assessment if the swallow is thought to be unsafe. $[\sqrt{ }]$

\section{AIRWAY CLEARANCE AND RESPIRATORY MUSCLE TRAINING}

Most independent airway clearance techniques start with the child taking a deep inspiration followed by a forced expiration (huff) or a cough. The deep breath gets air behind mucus (directly and via collateral ventilation), opens up the airway, loosens secretions in the small airways and mobilises the secretions to the larger airways. The secretions are then cleared from the central airways with an effective huff or cough. Independent airway clearance techniques are ineffective in children with significant NMW as they do not have the ability to take deep breaths, and they do not have an effective cough. Similarly oscillatory positive expiratory pressure devices are unsuitable for children with significant NMW since the child has to take a deeper than normal inspiration and breathe out against resistance while the device oscillates on expiration.

\section{Chest physiotherapy techniques in children with NMW}

Prior to chest physiotherapy inhaled treatment, including humidification, mucolytics, or hypertonic saline, may be beneficial to help promote secretion clearance.

\section{Standard chest physiotherapy}

Standard chest physiotherapy consists of postural drainage and manual techniques (percussion and vibrations). Chest percussion (chest clapping) is carried out using a hand, fingers or face mask and is generally well tolerated and widely used in small children and babies and may be effective in infants with NMW combined with nasopharyngeal suction. Chest vibrations involve the application of a rapid extra thoracic force at the beginning of expiration, followed by oscillatory compressions until expiration is complete. The compression and oscillation applied to the chest are believed to aid secretion clearance by increasing peak expiratory flow to move secretions towards the large airways for clearance by suction or a cough. Care needs to be applied with these techniques in infants when the chest wall is very compliant and the closing volume of the lung is high, as this can induce atelectasis. Greater benefit from standard chest physiotherapy may be achieved if the patient is able to take a deep breath. Deep breathing can be supported using noninvasive ventilators or by using intermittent positive pressure breathing (IPPB) devices.

\section{Intrapulmonary percussive ventilation}

Intrapulmonary percussive ventilation (IPV) is a modified method of IPPB which superimposes high-frequency mini bursts of air (100-300+ cycles per minute) on the individual's intrinsic inspiratory and expiratory breathing pattern. This creates an internal vibration (percussion) within the lungs and promotes secretion clearance.

\section{High-frequency chest wall oscillation}

High-frequency chest wall oscillation (HFCWO) can be provided by intermittent compression of the chest wall using an inflatable jacket or cuirass adjusted to fit snugly over the thorax, using frequencies of $5-20 \mathrm{~Hz}$. Vibration of the chest wall produces oscillatory airflow which in turn promotes mobilisation of secretions from the peripheral airways towards the mouth. With IPV and HFCWO there is the potential to mobilise a large volume of secretions and therefore it is important to ensure that the appropriate emergency equipment (eg, resuscitation bag and suction) is available in case of mobilising a large mucus plug into a central airway.

\section{Incentive spirometry}

IS devices are designed to mimic natural sighing or yawning by encouraging the patient to take long, slow, deep breaths. This is accomplished by using a device that provides patients with visual or other positive feedback when they inhale at a predetermined flow rate or volume and sustain the inflation for a minimum of $3 \mathrm{~s}$. The objectives of this procedure are to increase inspiratory volumes, improve inspiratory muscle performance, and re-establish or simulate the normal pattern of pulmonary hyperinflation. The device is often used in the postoperative period.

\section{Augmented cough}

Augmented cough methods are a key part of effective airway clearance in children with NMW. They have two componentsfirst to increase inspired volume and second to increase the expiratory forces applied to the inspired volume; both components can be shown to increase expiratory flow rates during coughing.

When bulbar muscle function is adequate to hold the glottis closed, inspiratory lung volumes can be increased using a number of techniques. The maximum insufflation capacity (MIC) is the maximum volume of air stacked within the patient's lungs beyond spontaneous vital capacity. MIC is attained when patients take a deep breath, hold their breath and then airstacking is applied using a bag-valve mask device, a volumecycled ventilator or glossopharyngeal breathing. When bulbar dysfunction prevents effective breath-holding, passive insufflation methods can be used. This can be achieved using an adapted bag-valve mask system with a closed expiratory port (lung volume recruitment bag), ${ }^{85}$ with MI-E devices, or with a volumecycled ventilator set to deliver a single inspiratory volume close to the predicted inspiratory capacity. ${ }^{86}$ When passive methods are used, the inspiratory volume achieved is called the lung insufflation capacity. Glossopharyngeal breathing consists of a series of 6-10 pumping strokes produced by the action of the lips, tongue, soft pallet, pharynx and larynx. Air is held in the chest by the larynx, which acts as a valve as the mouth is opened for the next breath. ${ }^{87} 88$ This represents the breathing pattern of a frog and is also known as 'frog breathing'. In addition to improve cough efficiency, glossopharyngeal breathing may have a role in enabling patients using daytime ventilation to have ventilator-free time. Some patients will have taught themselves to glossopharangeal breath to maintain self-ventilation during the day.

Expiratory airflow can be increased in children with NMW by compression of the chest wall or abdomen, sometimes called a manually assisted cough. Synchronous compression of the abdomen when the patient coughs causes a sudden increase in abdominal pressure; this causes the abdominal contents to push the diaphragm upwards, increasing expiratory airflow. ${ }^{89} 90$ This technique can be combined with air-stacking to achieve greater expiratory airflow during coughing.

MI-E devices clear secretions by applying a positive pressure to the airway (insufflation), then rapidly shifting to negative pressure (exsufflation). The rapid shift in pressure produces a high expiratory flow of 6-11 litres/s (360-660 litres/min), simulating a natural cough. 


\section{Evidence}

\section{Airway clearance methods during acute respiratory infection}

There are no controlled studies demonstrating the benefit of chest physiotherapy in children with NMW during acute respiratory deterioration associated with respiratory infection. Expert opinion and clinical experience strongly suggest that chest physiotherapy is a critical part of the management of this problem, and controlled studies with a placebo arm would not be ethical. In children with NMW who require invasive ventilatory support during an acute exacerbation, effective airway clearance is essential for resolution and successful extubation. Treatment protocols associated with a high success rate of extubation, which include aggressive airway clearance, have been published. ${ }^{39} 676891-94$

\section{Medium-term and long-term outcomes}

There are no controlled studies demonstrating the benefit of chest physiotherapy on important medium-term and long-term outcomes such as avoidance of acute respiratory failure during respiratory infection, preservation of lung function or survival. Observational studies and retrospective case reviews have shown decreased hospitalisations and increased survival in children with $\mathrm{NMW}^{39} 68$ 91-96 compared with historical controls. Improved outcomes have been associated with the introduction of treatment protocols which include effective airway clearance techniques. Families are provided with oxygen saturation monitors to use at home and if oxygen saturation falls below $95 \%$ in room air during a respiratory infection, airway clearance techniques (MI-E, air-stacking and manual assisted cough) are used until oxygen saturations are improved. Treatment protocols over the period of time when improved outcomes were observed also included use of NIV, and it is difficult to separate the benefit due to effective airway clearance from that due to NIV.

\section{Inhaled therapy (Dornase $\alpha$, mucolytics, hypertonic saline) and use of humidification}

No studies have evaluated the effects of inhaled therapies on secretion clearance in children with NMW. Children with NMW do not usually have underlying problems with mucociliary clearance or sputum rheology. No studies have evaluated the effect of humidification on airway clearance in children with neuromuscular disease using NIV.

\section{Oscillatory therapies}

The use of IPV was first reported in a case series of four patients (three children), three with NMW and one with atelectasis. Three patients demonstrated improvements in oxygenation and resolution of atelectasis on chest x-ray following IPV. One child required emergency cough assistance after IPV to clear a mucus plug from the trachea after the mobilisation of the secretions. ${ }^{97}$ A study of eight adults with DMD with tracheostomies compared IPV to forced expiratory techniques and manually assisted coughing. ${ }^{98}$ In five patients who had longstanding chronic hypersecretion (more than $30 \mathrm{ml}$ of airway secretions per day suctioned via a tracheostomy), sputum clearance was greater using IPV. Reardon and colleagues ${ }^{99}$ randomised 18 children (11-19 years of age) with neurological and neuromuscular disease to prophylactic IPV or IS for a 7-month period. Children in the IPV group had no pneumonia and no antibiotic use compared with three infections and 44 days of antibiotic use in the IS group. IPV was also associated with fewer total days in hospital for respiratory problems (none compared with 8 days) and fewer days absent from school.

There has been one study evaluating the effect of HFCWO. Twenty-three children with cerebral palsy $(n=9)$ and neuromuscular disease $(n=14)$ were randomised to treatment with HFCWO or conventional physiotherapy. ${ }^{100}$ Of the 14 patients with NMW, 10 were on nocturnal NIV. Patients were instructed to carry out the treatment that they were assigned to three times daily for an average of 5 months. Between the two groups there was no significant difference in any of their primary outcome measures (hospitalisation and antibiotic use). Caregivers reported a greater adherence to HFCWO rather than conventional physiotherapy. In a case report of a child with SMA type 1, HFCWO was found to be safe and seemed to assist in resolution of previously unresolved atelectasis on chest x-ray. ${ }^{101}$

\section{Incentive spirometry}

There has been one randomised controlled trial (described in the previous section on oscillatory therapies) in which IS was compared with intrapulmonary IPV in nine children with NMW during a hospital admission. The IS group had an increased length of hospital admission and an increase in the amount of antibiotic use. ${ }^{99}$

\section{Air-stacking}

Air-stacking to achieve MIC has been shown to increase CPF in children with SMA and DMD. ${ }^{86} 102-105$ Dohna-Schwake and colleagues $^{106}$ used IPPB via a mask or mouthpiece in a group of 29 children with NMW to achieve MIC. The mean pressure required for insufflation ranged from 22 to 40 mbar. The MIC increased from an unassisted inspiratory capacity of $0.68 \pm 0.40$ litres (mean \pm SD) to a MIC of $1.05 \pm 0.47$ litres $(p<0.0001)$. CPF rose from $119 \pm 58$ litres $/ \mathrm{min}$ to $195 \pm 74$ litres $/ \mathrm{min}(\mathrm{p}<0.0001)$ Patients with the lowest unassisted inspiratory capacity had greatest improvements in CPF. Lung insufflation capacity has been shown to increase CPF compared with an unassisted CPF in the presence of bulbar insufficiency. ${ }^{86}$ In one study of 11 children with SMA who were taught glossopharyngeal breathing, five were able to learn the technique with a subsequent range of improvement in vital capacity of $0-58 \% .{ }^{107}$ In another study glossopharyngeal breathing was achieved by $27 \%$ of 78 patients with DMD (mean age $20.6+3.1$ years). ${ }^{108}$ In the patients who were able to learn glossopharyngeal breathing, vital capacity increased from $244 \pm 151$ to $824 \pm 584 \mathrm{ml}(\mathrm{p}<0.001)$ and CPF from $164 \pm 76$ to $289 \pm 91$ litres $/ \mathrm{min}(p<0.001)$ and delayed the need for daytime ventilator use.

\section{Manual assisted cough}

Manual assisted cough is a simple effective technique that can be used anywhere and has been shown to increase CPF in children and adults with NMW compared with their unassisted CPF. $^{103-105} 109110$ Toussaint and colleagues ${ }^{110}$ studied 179 patients with NMW who were over 8 years old. Sixty-five per cent of the patient group had DMD and $60 \%$ of the total group were using NIV. Their findings showed that manual assisted cough alone could improve CPF in patients with a vital capacity between 1030 and $1910 \mathrm{ml}$ (or a maximum expiratory pressure of 14-34 $\mathrm{cm} \mathrm{H}_{2} \mathrm{O}$ ). Above or below these limits a manual assisted cough was unlikely to improve CPF.

\section{Air-stacking combined with manual assisted cough}

Several observational studies on adults and children with NMW have shown that combining air-stacking and manual assisted cough can lead to significantly greater improvements in CPF compared with use of either technique alone. ${ }^{103-105} 109110$ Toussaint and colleagues ${ }^{110}$ found that in patients with NMW who had a vital capacity of $<560 \mathrm{ml}$ (but $>340 \mathrm{ml}$ ) the combination of air-stacking and manual assisted cough was required to achieve a CPF above 180 litres/min. In patients with larger vital capacities air-stacking with or without manual cough 
assist could improve CPF, but the benefit decreased with increasing vital capacity.

\section{Mechanical insufflation/exsufflation}

There are no randomised controlled trials showing that mechanical insufflations/exsufflation (MIE) is more effective than other forms of physiotherapy in preventing respiratory exacerbation in children or adults with NMW. There are several studies which show that it is effective in increasing CPF. One study of 17 children with a range of neuromuscular disease ${ }^{111}$ demonstrated that MI-E using a range of inspiratory and expiratory pressures $\left(15-40 \mathrm{~cm} \mathrm{H}_{2} \mathrm{O}\right.$ ) was well tolerated by children with no effect on oxygen saturation during manoeuvre, and no complaints of abdominal distension, chest pain or discomfort. A significant increase in CPF was seen after using MI-E at $40 \mathrm{~cm} \mathrm{H}_{2} \mathrm{O}$ pressure. Another study of 22 patients (aged 10-56 years) with NMW compared the effectiveness of MI-E on increasing CPF with manual assisted cough or cough after a single inspiration supported by a non-invasive positive pressure ventilator. $^{112}$ The greatest increase in CPF was seen using the MI-E device (increase in CPF from 169+90 litres/min unassisted to 235 +111 litres/min with MI-E). No comparison has been made with air-stacking to MIC with or without manual cough assist.

The most effective pressures to use during MI-E are unclear. Some authors report better efficacy with higher pressure spans $\left(+40\right.$ to $\left.-60 \mathrm{~cm} \mathrm{H}_{2} \mathrm{O}\right),{ }^{39} 89919296111113$ while others report a good outcome with lower pressures ( +30 to $-30 \mathrm{~cm} \mathrm{H}_{2} \mathrm{O}$ and +20 to $\left.-30 \mathrm{~cm} \mathrm{H}_{2} \mathrm{O}\right){ }^{112}{ }^{114-116}$ Miske and colleagues ${ }^{115}$ reported the use of MI-E in 62 children (mean age 11.3 years) with NMW used for a mean duration of 13.4 months. MI-E was well tolerated in $90 \%$ of patients, with a resolution of chronic atelectasis in four patients.

MI-E has been used during chest infections in patients with NMW. In a crossover study of eight patients with NMW using NIV, use of MI-E significantly reduced treatment time compared with conventional chest physiotherapy with breathing support from the non-invasive ventilator (30 min for MI-E vs $47 \mathrm{~min}$ for conventional physiotherapy, $\mathrm{p}=0.03)$. The use of MI-E was accompanied by higher subjective feeling of fatigue. ${ }^{114}$ Vianello and colleagues ${ }^{116}$ compared 11 consecutive patients (two children) with NMW, who were admitted to the intensive treatment unit with a respiratory infection and who were treated with MI-E, with 16 historical controls (two children) who did not receive MI-E. Treatment failure (need for mini tracheostomy or intubation) was lower in the group treated with MI-E (2/11 vs $10 / 16, p<0.05)$.

Although pneumothorax is a potential risk from any positive pressure device there have been no reports of pneumothorax directly attributable to MI-E devices in children. There is a case report of two adult patients with NMW who were using MI-E and who developed pneumothoraces. ${ }^{117}$ There were other risk factors for pneumothorax in these patients, including the use of NIV and mild chronic obstructive lung disease.

\section{Evidence statements}

Use of airway clearance techniques which increase CPF, as part of a homecare treatment package which includes NIV, is associated with decreased hospital admission for respiratory infection and improved survival (evidence level 3).

Several airway clearance techniques are available. The most effective method for a particular child depends on the ability of the child to cooperate with treatment and the severity of the child's weakness (evidence level 3).

\section{Recommendations}

- Children with ineffective cough (including children over 12 years of age with CPF $<270$ litres/min), particularly if they have had episodes of deterioration with respiratory infection, should be taught augmented cough techniques. [C]

- Manual cough assist and air-stacking methods to achieve MIC are effective methods of improving cough efficiency and should be used when appropriate. [C]

- MI-E should be considered in very weak children, those with loss of bulbar function, and those who cannot cooperate with manual cough assist or air-stacking or in whom these methods are not effective. [C]

- Oscillatory techniques such as high-frequency chest wall oscillation and IPV should be considered in children who have difficulty mobilising secretions or who have persistent atelectasis, despite use of other airway clearance techniques. [D]

- Airway clearance techniques should be used during respiratory infection when oxygen saturation levels fall below 95\% while the child is breathing room air. If the techniques being used fail to result in an increase in oxygen saturation to $95 \%$ or above, different methods of airway clearance should be used. This may require attendance at hospital for treatment. [D]

- MI-E should be available in the acute setting in all hospitals that treat neuromuscular patients as an alternative method of airway clearance with the purpose of preventing deterioration and the need for intubation and mechanical ventilation. [D] Good practice points

- Nebulised normal saline may be considered in children who have continued tenacious secretions. [ $\sqrt{ }]$

- Humidification should be considered in children who use NIV and who have tenacious airway secretions. Care should be taken to ensure that use of humidification does not result in a troublesome increase in oral secretions. [ $\sqrt{ }]$

-When using sputum mobilising techniques, appropriate emergency equipment (eg, resuscitation bag and suction) should be available in case of mobilising large mucus plugs into the central airways where they may result in airways obstruction. [ $\sqrt{ }]$

- Children who use regular night-time or diurnal NIV should use their ventilator to support deep breathing during airway clearance treatments. Use of NIV during airway clearance sessions can help prevent respiratory muscle fatigue. [ $\sqrt{ }]$

- Children who use MI-E for airway clearance should be given a long enough periods of rest during treatment sessions to prevent respiratory muscle fatigue due to coughing. [ $\sqrt{ }]$

- At the end of a treatment session with MI-E it is important to complete the session with an insufflation to leave the child with an appropriate functional residual capacity. [ $]$ ]

\section{Respiratory muscle training}

Respiratory muscle training in normal subjects can lead to improvements in respiratory muscle strength and endurance. ${ }^{118}$ This observation has led to the application of respiratory muscle training to a number of disease states, including adults and children with NMW, with the aim of preserving or improving daytime and night-time ventilatory capacity and reducing respiratory exacerbations by improving cough efficiency.

Respiratory muscle strength is assessed by measuring maximal mouth inspiratory pressure and/or expiratory pressure the subject can generate. Respiratory muscle endurance is assessed by measuring the subject's maximum ventilation volume over a set period of time, typically 10-15 s (maximum voluntary ventilation), or by the duration for which a subject can breathe against a predetermined inspiratory load. Respiratory muscle strength training involves performing repetitive 
maximal, or near maximal, static inspiratory and/or expiratory manoeuvres against either a closed glottis or a nearly occluded resistor valve. Endurance training involves breathing through a variable resistor at a predetermined percentage of maximum capacity for a set period of time. Training programmes usually involve exercises for 10-20 min once or twice per day.

\section{Evidence}

There are no studies that have assessed the effects of respiratory muscle training in children or adults with neuromuscular disease on the clinically important outcomes of maintenance of ventilatory function over time, or on the reduction of respiratory exacerbations.

There are five randomised controlled studies of respiratory muscle training in a total of 107 children and adolescents with NMW which assessed changes in respiratory muscle strength and/or endurance as outcome measures. ${ }^{119-123}$ Most study subjects had DMD; the remainder had SMA type 2 or 3 . The training usually consisted of daily inspiratory and/or expiratory manoeuvres against a resistive load with a placebo arm using similar exercises without resistive loads. Training was carried out for 1-6 months. Three studies showed improvements in respiratory muscle strength and three studies showed improvements in respiratory muscle endurance. In one study the magnitude of improved respiratory endurance was correlated with total training time over a 6-week period. ${ }^{122}$ Duration of improvement in respiratory muscle strength was variable, returning to baseline 3 months after cessation of training in one study ${ }^{119}$ and persisting for 6 months in another. ${ }^{123}$ In the study by Wanke and colleagues, there was no improvement in respiratory muscle strength after 4 weeks training in a subgroup of five subjects who had a vital capacity of $<25 \%$ predicted and/or daytime hypercapnia. ${ }^{123}$ No studies showed an improvement in lung volumes associated with respiratory muscle training.

There are two longer-term observational studies ( 9 and 24 months' duration) assessing the effects of respiratory muscle training on mixed population of 43 children and young adults with DMD and SMA. ${ }^{124} 125$ Both studies showed sustained improvements in respiratory muscle strength and endurance while training was maintained. The study subjects had a wide range of baseline lung function (vital capacity 27-100\% predicted). Response to respiratory muscle training was not dependent on starting baseline vital capacity. There was no improvement in vital capacity associated with respiratory muscle training. Koessler and colleagues showed no fall in vital capacity over 2 years in their group of 27 boys with DMD. ${ }^{124}$ The absence of a control group makes interpretation of this observation difficult. There was no evidence of increased muscle damage caused by training in either study.

\section{Evidence statement}

Respiratory muscle training can improve respiratory muscle strength and endurance in children and young adults with DMD (evidence level 1-).

\section{ASSISTED VENTILATION}

Assisted ventilation is used in children with NMW for the following reasons:

- To treat the symptoms of nocturnal hypoventilation.

- To treat the symptoms of daytime hypoventilation.

- To reduce the frequency of hospital admission for chest infections.

- To prevent chest wall deformity in young children with the expectation of improved long-term outcome.

- To prolong life.

\section{Non-invasive ventilation}

Ventilation delivered via nasal mask, nasal pillows, face mask or mouthpiece is called NIV. NIV can be used to support breathing in children with NMW, at home and in hospital. Nasal masks or pillows and face masks are commercially available for all except the smallest infants. The mask is usually held in place with straps and a cap which fit over the head. Nasal masks or pillows are usually the most comfortable to use, and for most children will be the interface of first choice. Air leaks can occur through an open mouth when using nasal mask ventilation, and when this is a problem a chin strap or a mask covering the nose and mouth (face mask) may be needed. Nearly all children can be persuaded to wear a nasal or face mask at night for the purposes of ventilation, and once they become adapted to the ventilation will use it without difficulty. High compliance rates are usual, particularly when the child and the family appreciate that the NIV has led to symptomatic improvement. Untreated nocturnal hypoventilation can lead to daytime hypercapnia. Effective NIV which corrects nocturnal hypoventilation can also reverse daytime hypercapnia. The mechanisms by which nocturnal NIV improves daytime spontaneous ventilation are not fully understood; improved hypercapnic ventilatory response appears to be a consistent finding in studies on this topic. ${ }^{126-131}$

In children with more severe weakness, respiratory failure with hypercapnia can occur during wakefulness and during sleep to an extent that is not reversed by nocturnal ventilation alone. For these children, daytime NIV is used to relieve symptoms of hypoventilation, improve $\mathrm{QOL}$ and improve life expectancy. Daytime NIV can be delivered using the same nasal masks or pillows as those used for night-time ventilation. For older children who have sufficient motor and cognitive skills, mouthpiece ventilation may be preferred. Fitting a nasal mask or pillows for daytime ventilation requires a carer, and once on, may interfere with social interaction, speech and eating. Mouthpiece ventilation, using a harness to hold the mouthpiece close to the patient's face, allows the patient to control their ventilation and to take breaths as and when required. When children require ventilation for more than $16 \mathrm{~h}$ in each $24 \mathrm{~h}$ period, a tracheostomy may provide a more satisfactory interface between the child and the ventilator than a mask (see section on tracheostomy).

\section{Evidence}

\section{Correction of nocturnal hypoventilation and relief of symptoms}

The evidence that NIV is effective at correcting nocturnal hypoventilation and in alleviating the associated symptoms comes from one randomised study of a mixed population of children and adults ${ }^{78}$ and four non-randomised observational studies of children. ${ }^{130}{ }^{132-134}$ The children in the studies had NMW caused by a range of conditions, including DMD, SMA, congenital myopathies and congenital muscular dystrophies. All had nocturnal hypoventilation with or without daytime hypercapnia

The single randomised trial of NIV for nocturnal hypoventilation $^{78}$ included several school age children (mean age of participants 18 years, range $7-51$ years). The subjects were identified from a clinic population of patients with congenital neuromuscular or chest wall disease, with either symptoms of nocturnal hypoventilation or a vital capacity of $<50 \%$ predicted, and assessed by daytime blood gases and night-time oxygenation and carbon dioxide measurements. Those with the combination of nocturnal hypoventilation and daytime normocapnia were randomised to either nocturnal NIV or standard care and followed for 2 years. NIV was effective at correcting nocturnal hypoventilation and led to an improvement in QOL scores. Of 
note, 9 out of 10 patients in the control group were started on NIV by the end of the 2-year trial; two for relief of symptoms of nocturnal hypoventilation, five after they developed daytime hypercapnia, one because of poor growth and one for falling lung function.

In the four observational studies ${ }^{130}{ }^{132-134}$ a total of 91 children were commenced on NIV because of symptoms of hypoventilation, the presence of daytime hypercapnia, or following an episode of respiratory failure. In most children some form of physiological monitoring was available prior to commencing NIV. Three of the four studies assessed symptoms and QOL. Symptoms were assessed by questionnaire or parental recall. All four studies showed improvement in oxygenation and carbon dioxide levels during sleep and markedly improved respiratory disturbance index. Symptom scores were also significantly improved. In one study, ${ }^{130} 30$ children who had been established on NIV were asked to discontinue NIV for three nights. Eight families refused and 12 children made an attempt, but resumed NIV on the first night because of symptoms. Ten children completed three nights without NIV. In all 10 children there was a prompt return of nocturnal hypoventilation with hypercapnia. All five studies showed that nocturnal NIV was effective at reversing daytime hypercapnia.

There has been one randomised trial of early NIV, used to try and prevent progression to daytime respiratory failure. ${ }^{135}$ This was carried out in boys with DMD (mean age 15.5 years) who were considered not to require immediate NIV on the basis of having a vital capacity $>20 \%$ predicted and a normal daytime arterial carbon dioxide level. They were randomised to NIV or conventional treatment and followed for 5 years. The primary endpoint of the study was survival. There was no survival benefit in the group using NIV; there were eight deaths (five from respiratory failure) in the NIV group compared with two deaths (one from respiratory failure) in the control group. This study has a number of drawbacks. It does not comment on whether patients had symptomatic nocturnal hypoventilation or whether this was corrected by NIV. No overnight oximetry or capnography was carried out. Compliance with NIV was ascertained by patient and parental report only. Conventional care was said to include the use of antibiotics and physiotherapy, but details are sparse, and specific interventions to improve cough and secretion clearance are not discussed. This study does suggest that provision of NIV in those who are predominantly asymptomatic, without careful monitoring of its effectiveness and without attention to other aspects of general and respiratory health (in particular to airway secretion clearance), is not likely to be successful.

\section{Evidence statements}

NIV is effective in correcting sleep-disordered breathing and daytime hypercapnia (evidence level 1-).

NIV is effective in alleviating the symptoms of nocturnal hypoventilation (evidence level 1-).

Preventive use of NIV in children with DMD does not alter progression to respiratory failure (evidence level 1-).

\section{Reduction in hospital admission}

The evidence that use of NIV reduces the frequency of hospital admission comes from three small retrospective studies. ${ }^{134} 136137$ Combined together the studies included 59 children with NMW started on NIV for documented nocturnal hypoventilation, daytime hypercapnia, following acute deterioration or because of recurrent hospital admissions with respiratory exacerbations. All three studies compared the rate of hospital admission in the year preceding NIV to the year following commencement of NIV. All three studies showed reduction in the frequency of admission (2-4 per year pre NIV vs 1 per year post NIV) and total hospital days (40-50 per year pre NIV vs 10 per year post NIV) in the year following commencement of NIV. The studies are retrospective and it is likely that factors other than NIV also contributed to the reduction in hospital admission, for example, increased use of effective chest physiotherapy for clearance of airway secretions. Tzeng and $\mathrm{Bach}^{39}$ report on the use of a protocol approach for acute respiratory exacerbations, including the use of NIV in a retrospective study of 94 adults and children, including 31 with DMD (mean age 19 years) and 18 with SMA (mean age 7 years) Use of the protocol was also associated with reduction of hospital admission (approximately one admission per year pre protocol vs 0.1 admissions per year when protocol used).

\section{Evidence statement}

NIV is effective in reducing hospital admissions for respiratory exacerbations in children with either symptomatic sleep-related hypoventilation or daytime hypercapnia (evidence level 3).

\section{Prevention of chest wall deformity}

Chest wall deformity, typically pectus excavatum, is common in children with severe forms of SMA. This is thought to arise as a result of the action of the relatively spared diaphragm on the weak chest wall. Respiratory movements are asynchronous with diaphragmatic descent causing outward movement of the abdomen and in-drawing of the lower chest. Initially the bellshaped chest shape is reversible with positive pressure ventilation, but in the longer term pectus excavatum can become a fixed deformity. Avoidance of chest wall deformity may preserve larger passive lung volumes in later life, potentially allowing easier ventilation and better CPFs. The evidence that NIV can prevent chest wall deformity is based on two observational studies of infants with SMA. ${ }^{96} 138$ In these studies chest wall deformity could be prevented or reversed by use of highspan NIV using median peak pressures $18 \mathrm{~cm} \mathrm{H} \mathrm{H}_{2} \mathrm{O}$. Titrating NIV pressure so that NIV not only corrects nocturnal hypoventilation, but also abolishes asynchronous chest and abdominal movements, may be a useful assessment of the effectiveness of NIV in preventing longer-term chest wall deformity. ${ }^{139}$

\section{Evidence statement}

NIV may be effective in preventing chest wall deformity in children with SMA (evidence level 3).

\section{Increased life expectancy}

The evidence that NIV results in prolongation of life in children with life-limiting NMW comes from seven studies of children with either DMD or SMA. ${ }^{36} 93-95140-142$ Four studies describe significantly improved survival of boys with $\mathrm{DMD}$ compared with historical controls. Improved survival was attributed to routine use of NIV, although more effective airway clearance interventions are likely to have contributed. One study ${ }^{36}$ described the clinical course of 10 patients with DMD with daytime hypercapnia (mean age 20.2 years). Five patients were treated with NIV while the other five refused the intervention. At the end of the 2-year follow-up period, four out of the five patients who declined the use of NIV had died of respiratory failure, while all five of the ventilated group were still alive. Two studies $^{9495}$ describe the outcome of children with SMA type 1, one based on a questionnaire survey sent to 391 families of affected children and one based on a retrospective review of 92 children attending a single clinic. Both showed that improved survival was associated with use of mechanical ventilation (via mask or tracheostomy). 


\section{Evidence statement}

Use of NIV increases life expectancy in children with lifelimiting NMW (evidence level 3).

\section{Use of NIV for daytime ventilation}

The evidence that daytime ventilation can be delivered effectively via a mask or mouthpiece (rather than a tracheostomy) comes from three observational studies-two in patients with DMD (a total of 66 patients) ${ }^{143} 144$ and one in children with SMA type 1 (47 children from one centre). ${ }^{94}$ In these studies patients with daytime ventilation supported with NIV had similar long-term survival compared with patients supported with tracheostomy ventilation.

\section{Evidence statement}

Daytime ventilation by mouthpiece or nasal mask can be effective in controlling symptoms of daytime hypoventilation (evidence level 3).

\section{Recommendations}

- Children with NMW resulting in symptomatic nocturnal hypoventilation or daytime hypercapnia should be supported with NIV. [C]

- A non-invasive approach should be considered in children needing daytime ventilation. [D]

\section{Ventilator types, modes and set up Ventilator types}

There is a wide range of home ventilators suitable for use in children with NMW. The two main ventilator types are pressure-targeted ventilators and volume-targeted ventilators. Some (more expensive) hybrid machines can operate in volumetargeted and pressure-targeted modes, and some can deliver a pressure-targeted volume-assured mode. There are no longterm studies comparing clinically important outcomes (symptom control, hospital admission, survival) for different modes of ventilation.

Early home ventilation devices were volume targeted, and delivered a preset tidal or minute volume, and volume-targeted machines are still preferred by some centres. A major benefit of volume-targeted machines is the ability to use the ventilator to assist with air-stacking as part of assisting coughing techniques. Although volume-targeted ventilators have the potential to deliver known tidal volumes, most machines have a limited capacity to correct for leaks (either around the mask or through the mouth if a nasal mask is used) and this can lead to underventilation.

Pressure-targeted ventilators tend to be lighter and cheaper than volume-targeted machines. Pressure-targeted ventilators are designed to increase flow until peak pressure is reached, which means that they effectively compensate for all but the largest leaks. Pressure-targeted ventilators use a single limb circuit with exhaled gas usually being vented through ports near the face or nasal mask. There is the potential to re-breathe carbon dioxide if airflow is low during expiration, ${ }^{145}$ and for this reason, a minimum end expiratory pressure of $4 \mathrm{~cm} \mathrm{H}_{2} \mathrm{O}$ when using pressure-targeted ventilation is advisable. If hypercapnia persists despite otherwise adequate ventilation, and is thought to be related to re-breathing of carbon dioxide, a low-resistance expiratory valve can be added to the circuit.

\section{Ventilator modes}

A variety of different modes of ventilation are available using pressure-targeted and volume-targeted ventilators. There are two common modes used in neuromuscular patients; one whereby the patient can trigger a supported breath, but cannot control the inspiratory time - this mode is variously called spontaneous/ timed, assist/control or pressure-control ventilation; and one whereby the patient can trigger a supported breath and control the length of the breath-this mode is called pressure-support ventilation. The purpose of having different modes is to try and maximise patient-ventilator synchrony and patient comfort and to minimise respiratory work. During pressure-support ventilation, inspiration continues after a breath is triggered or initiated by the ventilator until inspiratory flow falls to a preset level, or for a maximum of $3 \mathrm{~s}$. This can be the most comfortable mode of ventilation in adults, but is not suitable for most small children with small tidal volumes. Fauroux and colleagues ${ }^{146}$ bench-tested the performance of 17 ventilators used for home NIV, using a lung model. The sensitivity of the expiratory (and inspiratory) trigger of most of the ventilators was ineffective for infants and for older children when there was a leak in the circuit. The inability of the ventilator to detect the child's expiratory effort will result in long and uncomfortable inspirations. For these children ventilator modes with a fixed inspiratory time are more effective and better tolerated.

Although the need for a back-up rate has not been demonstrated by clinical trials, clinical experience shows that most children with NMW who are effectively ventilated during sleep will breathe at the back-up rate, with ventilator-initiated, rather the patient-triggered breaths.

\section{Starting NIV}

In children with clinically stable conditions, nocturnal NIV can be established gradually in the home setting over a period of several nights, building up the time the child uses on the ventilator, with support usually from a specialist nurse practitioner. For many children the indication for NIV will be symptomatic hypoventilation, and relief from these symptoms is usually sufficient to persuade both the child and the parents of the value of continuing with the treatment. It is therefore important that if the initial pressure or volume settings chosen are low, to promote tolerance to the ventilator, they are increased quickly to those likely to provide effective ventilation.

\section{Evidence}

There is one study that has compared the effectiveness of NIV started in the inpatient setting to that started in the outpatient setting. ${ }^{147}$ This was a randomised trial of 28 children and adults starting NIV for symptomatic nocturnal hypoventilation. Fourteen patients started NIV as inpatients (median length of hospital stay was 4 days) and 14 as outpatients (one or two outpatient visits). All patients received follow-up advice by telephone. When assessed 2 months later, there was no difference in improvement of nocturnal gas exchange, compliance with ventilator use, or QOL between the two groups.

\section{Evidence statement}

Children with stable disease can be started on NIV without need for admission to hospital (evidence level 1-).

\section{Good practice points}

- Clinical teams caring for children using home ventilators should become familiar with a small number of machines. For most children pressure-targeted machines work well and are simple to use. $[\sqrt{ }]$

- Ventilation modes with fixed inspiratory times are usually the most appropriate for use in young or very weak children. $[\sqrt{ }]$

- For older teenage children who need to use mouthpiece ventilation during the day, and/or who use air-stacking as a way of assisting cough, volume-targeted or hybrid ventilators may be preferred. [ $\sqrt{ }]$ 
- When full face masks are used, anti-asphyxia valves should be fitted to allow room air breathing in case of ventilator failure. The risks of the child vomiting and aspirating should be considered, particularly if the child is unable to remove the mask on their own. [ $\sqrt{ }]$

- Children needing ventilatory support for more than $16 \mathrm{~h}$ per day should be provided with two ventilators in case of equipment failure. [ $\sqrt{ }]$

\section{Monitoring children using NIV}

Irrespective of the circumstances in which NIV is started, the primary purpose of NIV is to correct hypoventilation and the associated symptoms. Effectiveness of NIV will vary according to several factors, including the age of the child, the compliance of the lungs and chest wall, and the fit of the mask. Once the child is able to tolerate NIV throughout the night (a process that usually takes two to three nights), monitoring should be carried out to check that hypoventilation has been abolished. The minimum requirement is a sleep study recording continuous oximetry and capnography. When correction of thoraco-abdominal asynchrony is desirable (with the expectation that it will prevent long-term chest wall deformity), then a sleep study which includes chest and abdominal movement analysis in addition to oximetry and capnography will be needed. Adjustments of NIV settings may be necessary to achieve the desired outcome. Reported symptomatic improvement alone is not a reliable means of assessing the effectiveness of NIV - partial correction of nocturnal hypoventilation may lead to incomplete symptom improvement.

The frequency at which further monitoring should be carried out depends on the clinical circumstances. Newly diagnosed infants with profound weakness may need to be seen every few weeks. In older stable children with slowly progressive or nonprogressive disease, annual assessment is sufficient.

NIV will not work unless it is being used. Adherence to NIV in children, who will be supported by their parents, is not usually a problem. Many ventilators have a timer which indicates hours of use, and this can be used to assess adherence. When discussing issues of adherence, it is important to remember that for many children the primary purpose of NIV is relief of symptoms.

\section{Leaks}

Leaks are inevitable when delivering ventilation via mask interfaces and are a common reason for NIV to be ineffective and to be poorly tolerated. Leaks around the mask can be minimised by careful mask selection. There is now a wide variety of paediatric masks, including nasal pillows, nasal masks or full face masks. The choice of interfaces for infants remains limited. Mouth leaks when using a nasal mask can be reduced either by a chin strap or by switching to a full face mask.

\section{Humidification}

Humidification is generally not required in children using NIV at night-time only. In children using NIV during the day and at night, humidification may be helpful in preventing drying of secretions and may improve tolerability. The most effective humidification is provided by heated tube humidification systems.

\section{Evidence}

There are no studies on the optimum frequency and nature of monitoring for children using NIV.

Three studies in adults report the effects of leaks in patients using nocturnal ventilation. ${ }^{148-150}$ Air leaks were found in all patients, and in one study of NIV volume-targeted ventilation an average of $25 \%$ of the ventilator-delivered breaths were lost to air leak. In another study, switching patients to ventilators with leak adjustment led to improvements in morning arterial carbon dioxide levels. In a small study of six adults using pressuretargeted ventilation, 60-90\% of arousals during sleep occurred after a period of leak, and the leak stopped temporarily after the arousal. Oxygen saturation was maintained by leak compensation in all but one patient, suggesting mechanisms such as increased airflow were the cause of the arousal rather than hypoxaemia.

\section{Evidence statement}

Air leaks are common in patients using nasal mask NIV and can result in incomplete correction of nocturnal hypoventilation and sleep disturbance (evidence level 3).

\section{Recommendations}

- Once a child has become established on NIV, a sleep polygraphy or oxycapnography should be carried out to check that it has effectively abolished sleep-associated hypoventilation. Ventilator settings should be adjusted and rechecked as necessary. [D]

- Children supported by NIV should be assessed regularly with repeat sleep studies to ensure continued effectiveness of NIV at preventing hypoventilation. The frequency of review will vary according to clinical circumstances, but should not be less than every 12 months. [D]

\section{Adverse effects of NIV}

\section{Skin breakdown}

Break down of the skin, usually on the bridge of the nose or forehead, can occur when using nasal or face-mask ventilation. Prevention is better than cure, and careful attention to mask fitting, and avoidance of over-tight straps is usually sufficient to keep the contact areas healthy. It is better to allow a small leak around the mask, than to have it overly tight. When red patches are seen after mask use, these should be dealt with promptly before the skin breaks down. Use of wound care tape, particularly those made of hydrocolloid material, is often helpful. Forehead spacers can also reduce the pressure of the mask on the nasal bridge. A wide range of commercial masks are now available for older children and changing the mask to one which avoids the area of skin damage is usually possible. This is more difficult for infants in whom there is a much more limited choice of interfaces. Custom-made masks are associated with a lower incidence of skin injury. ${ }^{151}$

\section{Mid-face hypoplasia}

Pressure from the nasal or face mask used for NIV on the growing face can result in under-development of the maxilla, leading to mid-face flattening and mal-occlusion of the teeth. There are no large studies indicating the incidence of this problem, nor how it should be managed. Fauroux and colleagues ${ }^{151}$ report a survey of 40 children using NIV or CPAP for at least 4 weeks. Global facial flattening was seen in $68 \%$ of children. Twleve per cent were described as having a concave facial appearance. Facial flattening did not appear to be associated with type of mask, age or duration of use. Maxillary retrusion was seen in 37\% of children, and this was associated with how many hours the mask was worn each day. One case report describes how a nasal mask was successfully modified to allow orthodontic treatment in a 7-year old girl who has used nocturnal NIV from the age of 9 months. ${ }^{152}$ Full face masks which avoid pressure on the maxilla may also be effective in managing maxillary retrusion.

\section{Good practice point}

- Assessment for skin injury and facial flattening should be carried out regularly in children using NIV and the mask interface adjusted as necessary to minimise these complications. [ $\sqrt{ }]$ 


\section{Tracheostomy}

Ventilation at home can be delivered via a mask interface, a mouthpiece or a tracheostomy. Tracheostomy for home ventilation was frequently used in the past before there were mask interfaces suitable for children. With currently available ventilators and mask interfaces, NIV is the mode of choice for the vast majority of children with NMW needing respiratory support during sleep.

\section{When should a tracheostomy be considered?}

- Severe bulbar dysfunction. When there is severe bulbar dysfunction resulting in frequent aspiration, tracheostomy may be necessary to allow more effective airway clearance.

- Failure to extubate. After an acute exacerbation has led to a period of invasive ventilation and extubation has failed despite optimal management for 2 weeks or more.

- When ventilatory support is needed for more than $16 \mathrm{~h}$ per day.

- When there is failure to correct hypoxaemia or hypercapnia with NIV.

- Severe mid-face hypoplasia not correctable by adjusting the NIV interface.

The preferences of the family, child or young person and the experience of the clinical team need to be taken into account when making this decision. Tracheostomies have the advantage of leaving the face free, making social interaction and eating easier and providing direct access for suction. They also provide a more secure interface for patients who are ventilator dependent.

\section{Tracheostomy complications}

Use of tracheostomy in young children with severe NMW can result in immediate loss of autonomous breathing, possibly because loss of laryngeal function results in an inability to maintain an effective functional residual capacity. Very weak children with tracheostomies are also less likely to be able to speak than those supported on NIV. In surveys of adults needing daytime ventilation some clearly prefer NIV to tracheostomy (the converse is also true), and tracheostomy ventilation may be associated with more respiratory symptoms and more frequent hospital admission than similar patients using NIV.

Adverse effects of tracheostomies include increased respiratory secretions and respiratory infections, dysphagia, granuloma formation and tracheo-arterial fistulas with catastrophic haemorrhage. ${ }^{153} 154$ Most of these problems can be minimised by using a well fitting flexible tracheostomy tube, centred in the tracheal lumen. Tracheostomy tubes are more likely to rub against the tracheal wall when the airway is distorted, such as in children with scoliosis. Tracheal erosions can be exacerbated by unsupported ventilator tubing pulling on tracheostomy tubes and inappropriate tracheal suction. Flexible endoscopy can be used to check that end of the tracheostomy tube is centred in the trachea and that the trachea is healthy.

\section{Evidence}

There are three studies that compare outcomes in patients with diurnal respiratory failure supported by tracheostomy or NIV. Soudon and colleagues ${ }^{144}$ compared the clinical course of 16 patients with DMD managed with tracheostomy ventilation (mean age 32.7 years) with that of 26 patients using NIV (mean age 27 years). Over a 5 -year period there was no difference in mortality. Sixty per cent of the patients with tracheostomy had problems related to the tracheostomy-mainly related to suction and granuloma formation. Patients in the tracheostomy group had more chest infections $38 \%$ in the tracheostomy group vs $8 \%$ in the NIV group). Loss of weight and need for gastric feeding appeared less frequent in the tracheostomy group. Bach and colleagues ${ }^{155}$ carried out a postal questionnaire of 654 adult patients using home ventilation. Of the patients with $\mathrm{DMD}, 33$ were using NIV for more than $16 \mathrm{~h}$ per day and 24 were ventilated via a tracheostomy. There was a lower rate of hospital admissions in the patients using NIV $(0.2$ admissions per patient per year vs 0.6 admissions per patient per year; $p<0.05)$. Similar results were seen when comparing NIV with tracheostomy ventilation for patients with other forms of myopathy. There were no apparent differences in bulbar function or duration of ventilator use between those using tracheostomy ventilation and NIV.

Bach and colleagues ${ }^{94}$ describe the outcome of 74 infants with SMA type 1 in a non-randomised study. Forty-seven children were managed with NIV and 27 had tracheostomies. Patient care was otherwise similar between the two groups, including provision of home oximeters and mechanical cough assist devices, with appropriate training and protocols for their use. The followup period was a mean of 5 years. Twenty-five out of the 27 patients with tracheostomy lost autonomous breathing immediately after the tracheostomy procedure. Of the 47 patients using NIV, 32 used it only during sleep, 6 required it for more than $16 \mathrm{~h}$ /day and became hypercapnic and dyspnoeic when not using it, and 9 required it continuously, with little or no breathing autonomy. All patients required NIV continuously during upper respiratory tract infections. Mortality in the two groups was similar (5/27 in the tracheostomy group and 8/47 in the NIV group), most attributable to sudden death at home or related to respiratory infection. Hospitalisation was more frequent in the NIV group in the first 3 years -1.6 admissions per patient per year compared with 0.4 admissions per patient per year in the tracheostomy group. After the age of 5 years hospital admission was unusual in either group ( 0.1 admissions per patient per year). The 47 children in the NIV group underwent a total of 194 intubations for respiratory failure-a substantial burden. More of the children in the NIV group could communicate verbally (34/47 vs $6 / 27$ in the tracheostomy group).

Two studies have assessed patient choice with respect to NIV or tracheostomy ventilation. Bach ${ }^{156}$ reported the findings from 168 adults who used home ventilation (mean age 55 years), most of whom were polio survivors with non-progressive disease, who had used both tracheostomy ventilation and NIV, each for at least 1 month. At the time of the survey 111 patients were using tracheostomy ventilation and 59 were using NIV. Of the 111 patients using tracheostomy ventilation, 49 expressed a preference for NIV, 42 for tracheostomy and 14 had no preference. Of the 59 patients using NIV, all preferred NIV over tracheostomy ventilation. Markstrom and colleagues ${ }^{157}$ assessed QOL by postal questionnaires in 91 adults (mean age 59 years) using home ventilation for restrictive lung disease, including 16 patients with neuromuscular disease. Sixty patients used NIV and 31 used tracheostomy ventilation. The QOL scores, using two out of three different measures, were no different between the NIV and tracheostomy groups. For the third measure of $\mathrm{QOL}$ there was a significantly better score in the tracheostomy group, but the difference between the groups was small (NIV group score $25.2 \pm 3.6$ compared with $27.8 \pm 3.7$ in the tracheostomy group; possible scores range from 9 to 36 ).

\section{Good practice points}

- Tracheostomy tubes should be carefully sized and sited to ensure the tip of the tube does not abut the tracheal wall. $[\sqrt{ }]$

- Family and child preference should be taken into account when considering tracheostomy to facilitate diurnal ventilation. $[\sqrt{ }]$ 


\section{Negative pressure ventilation}

Negative pressure ventilators, including iron lungs, cuirass ventilators and wrap ventilators (a rigid plastic thoracic cage and a nylon poncho), and abdominal displacement ventilators such as the rocking bed and the pneumobelt were widely used between 1920 and the late 1980s. There are reports of negative pressure ventilators being used in patients with NMW, including those with $\mathrm{DMD} .{ }^{158}$ The devices were effective at correcting night-time ventilation, although in weaker patients upper airway obstruction during sleep limited their effectiveness. The devices tended to be cumbersome and uncomfortable and have now been almost entirely superseded by modern positive pressure ventilators.

A modern version of the negative pressure cuirass is available commercially, and can be used to support breathing in children and infants with NMW, particularly those with diaphragmatic weakness. It can be useful as an adjunct to NIV for short periods of time, including use when weaning children from positive pressure ventilation. An appropriate fit may be difficult in children with severe thoracic scoliosis.

\section{Evidence}

There are no studies on the use of negative pressure ventilation in children with neuromuscular disease.

\section{Oxygen}

Recurrent desaturation during sleep occurs in children with NMW and nocturnal hypoventilation. While clinical teams familiar with this problem will quickly recognise that ventilatory support is required, less experienced teams may believe that supplementary oxygen given during sleep would be helpful. This situation may arise in children with unrecognised nocturnal hypoventilation who are admitted to hospital for surgical procedures or for treatment of chest infection, and are found to have night-time desaturation during admission. Use of oxygen alone in these situations can worsen alveolar hypoventilation. If the child is normocapnic and normoxic during wakefulness, commencing ventilatory support is not urgent, and appropriate referral can be made if this service is not available in the child's local hospital.

\section{Evidence}

There is only one study on the use of oxygen therapy in patients with hypoventilation due to $\mathrm{NMW}^{159}$ This was an observational study of seven young adults with DMD with normal daytime blood gases. Six subjects had significant desaturation episodes at night, mainly during REM sleep. Oxygen therapy was effective at preventing desaturation in five out of six subjects. In all subjects use of oxygen was associated with a significant increase in the duration of REM-associated apnoea and hypopnoea.

\section{Evidence statement}

Although low flow oxygen overnight can reduce sleep hypoxaemia in subjects with nocturnal hypoventilation, it is associated with prolongation of underlying sleep hypopnoea and apnoeic events (evidence level 3).

\section{Good practice point}

- Oxygen alone should not be used to correct hypoxaemia caused by hypoventilation in patients with neuromuscular disease. $[\sqrt{ }]$

\section{Acute respiratory failure}

Acute respiratory failure in children with NMW is usually precipitated by viral respiratory infection; onset is usually over a few days, with increasing respiratory secretions and increasing respiratory work. Without intervention, the child may tire, with worsening of carbon dioxide retention and decompensation. Respiratory failure may also occur after surgery, particularly scoliosis surgery, usually because of pain or increased airway secretions associated with tracheal intubation. Successful management of acute respiratory failure requires anticipation and early intervention. Effective airway clearance is essential; methods that can be used are described elsewhere in the guideline. General supportive care is also important, including maintenance of fluid balance and nutrition. Increased respiratory work in children with NMW, and especially those with SMA, may result in metabolic acidosis, increased respiratory drive and worsening fatigue. NIV can support children at risk of respiratory failure and prevent the need for intubation. In children who have been intubated, either for respiratory failure or for the purposes of surgery, NIV can successfully facilitate extubation. Extubation is more likely to be successful if airway secretions are minimal, supplemental oxygen is not required and effective airway clearance methods are used before and after extubation. Children who are immediately supported with NIV after extubation will be less likely to tire and require re-intubation.

\section{Evidence}

The evidence that NIV can be used to manage acute respiratory failure without the need for intubation in children and young people with NMW comes from five observational studies on a total of 55 subjects, age range 3 months to 69 years, 48 known to be under 25 years. ${ }^{160-164}$ In 46 of the 60 episodes of acute respiratory failure affecting patients under 25 years of age, NIV was successful and intubation was not required. In one of the studies, ${ }^{162}$ NIV was combined with cricothyroid mini-tracheostomy to facilitate secretion clearance. Failure of NIV was associated with bulbar dysfunction and difficulty clearing airway secretions. The methods used for airway clearance methods are poorly described, and the impact of effective airway clearance, for example, with mechanical cough assist devices, on the success rate of NIV in the management of acute respiratory failure is not known.

The evidence that NIV can facilitate extubation in children with NMW comes from three observational studies on a total of 25 children (age 2 months to 17 years). ${ }^{92} 161165$ The majority of the children had been intubated and ventilated following an episode of acute respiratory failure; two had been intubated as part of anaesthesia for surgery. Twenty-two of these children were successfully extubated using NIV either as a transition to self-ventilation or to long-term nocturnal use of NIV for respiratory support. Bach and colleagues ${ }^{92}$ describe a protocol for the management of intubated children with severe muscle weakness. The key elements of this protocol are limited requirement for suction and no requirement of supplemental oxygen prior to extubation, frequent airway clearance using MI-E before and after extubation, and extubation to continuous NIV. Nine children with severe SMA had 28 episodes of respiratory compromise resulting in 48 intubation events. Extubation using conventional weaning methods was successful in two out of 20 attempts compared with 23 out of 28 attempts using the protocol $(p<0.001) .{ }^{92}$ The same protocol has been successfully used in a 157 adults and children with a wide range of myopathic conditions, including 25 with SMA and 20 with DMD. ${ }^{67}$ These subjects were unable to pass conventional trials of spontaneous breathing prior to extubation.

\section{Evidence statements}

NIV is a safe and effective therapeutic option for treatment of acute respiratory failure in children with neuromuscular diseases (evidence level 3). 
NIV can facilitate endotracheal extubation in children with NMW (evidence level 3).

\section{Recommendation}

- NIV should be the first-line treatment for children with NMW in acute respiratory failure. [D]

\section{Good practice point}

- Intensive care units caring for children with NMW should be aware of appropriate extubation criteria. These should include the presence of only minimal airway secretions, use of effective airway clearance methods (such as MI-E devices) and oxygen saturation more than $94 \%$ without supplemental oxygen for more than $12 \mathrm{~h}$. Continuous NIV should be used immediately after extubation. [ $\mathrm{V}]$

\section{PLANNING FOR SURGICAL PROCEDURES}

Children with NMW are vulnerable to the adverse effects of general anaesthesia and surgery and have increased intraoperative and postoperative morbidity. Respiratory muscle strength is diminished in the postoperative period, most often due to the combined effects of pain and sedation, and can result in hypoventilation and airway secretion retention. With proper planning major surgery can be successfully carried out in children with severe NMW, including those using non-invasive ventilatory support.

Preoperative assessment is recommended to identify children with NMW at risk of adverse outcome after surgery; risk factors include daytime and/or night-time hypoventilation, vital capacity $<60 \%$ predicted and ineffective cough. Cardiac assessment is important in children with conditions that involve cardiac muscle, such as DMD. Nutritional status should also be reviewed. In very high-risk children, it may be appropriate to discuss with the family the risk of prolonged mechanical ventilation after surgery, and in some circumstances, the possibility of tracheostomy. Preoperative training using airway clearance techniques and in the use of NIV may be helpful if it is anticipated that either may be needed in the postoperative period. NIV is often most effective when used immediately after extubation (see section on acute respiratory failure); the alternative of waiting to see if the child can manage without support can lead to exhaustion, secretion retention and the need for reintubation. Specific protocols (see section on acute respiratory failure) can improve the success of extubation of children with NMW, particularly for those:

- who already use NIV (usually for nocturnal hypoventilation)

- who have a previous history of failed extubation

- with an ineffective cough-including children over 12 years of age who have a CPF of $<270$ litres/min

- with vital capacity $<60 \%$ predicted.

Options for respiratory support of children with neuromuscular conditions during sedation or under anaesthesia include laryngeal mask airway, NIV delivered via nasal mask, manual ventilation using an anaesthetic bag attached to a nasal or face mask, or intubation. The choice of respiratory support for an individual patient undergoing anaesthesia depends on the preferences, experience and skills of the medical team involved. Malignant hyperthermia-like reactions and rhabdomyolysis are associated with the use of inhaled anaesthetics in children with many neuromuscular diseases including $\mathrm{DMD}$, myotubular myopathy and myotonic dystrophy. ${ }^{166-168}$ Cardiac arrhythmia is a well documented complication of anaesthesia in muscular dystrophy, particularly DMD. This may be precipitated by inhaled anaesthetic agents such as halothane and by succinylcholine. ${ }^{169}$ Safe anaesthesia in children with NMW requires suitably trained and experience anaesthetists.
In the postoperative periods the following additional precautions should be noted:

- Hypoxaemia can be caused by atelectasis, hypoventilation and retained airway secretions. Supplemental oxygen should be used with caution, as hypercapnia can be missed and may result in delay in treating the underlying cause. Airway clearance methods using assisted cough techniques with or without NIV are likely to be required.

- Fluid balance and cardiac monitoring are particularly important for patients such as those with $\mathrm{DMD}$, who are at risk of congestive heart failure and dysrhythmias.

- Children with NMW are at increased risk of respiratory depression associated with opiate analgesics. Epidural catheter analgesia may be a safer and more effective method of gaining pain control. ${ }^{170}$

- Children with neuromuscular disease are prone to gut dysmotility. The use of pro-kinetics and gastric decompression with a fine-bore nasogastric tube can avoid the problem of gastric distension impinging on the diaphragm and causing respiratory embarrassment.

- Nutritional support may be necessary to avoid malnutrition and further weakening respiratory muscles. ${ }^{171}$

\section{Evidence}

There is no published evidence that preoperative assessment reduces postoperative complications.

\section{Vital capacity as an indicator of respiratory morbidity}

The evidence that vital capacity can predict risk of postoperative respiratory complications comes from four retrospective studies of a total of 173 children with NMW, mainly DMD, undergoing scoliosis surgery. ${ }^{172-175}$ In all four studies the likelihood of remaining intubated and ventilated for more than 3 days after surgery was higher in children with lower preoperative vital capacity. In one study of 48 teenagers with $\mathrm{DMD}$, all boys with a preoperative vital capacity of more than $50 \%$ predicted were extubated immediately after surgery without complications. ${ }^{172}$ In another study of 125 children with scoliosis, 57 of whom had neuromuscular disease, a preoperative vital capacity of $<60 \%$ predicted the need for prolonged ventilation ( $>3$ days) with sensitivity of $77 \%$ and specificity of $56 \% .{ }^{174}$

A low vital capacity does not preclude good outcome from surgery. One descriptive study reports successful extubation immediately after scoliosis surgery in 14 consecutive subjects with DMD with preoperative vital capacity of $<30 \%$ predicted. ${ }^{176}$ In this study all 14 boys were admitted to hospital 6 weeks prior to surgery and underwent daily inspiratory muscle training, which appeared to result in a small increase in vital capacity: from $21.6 \%$, range $16-27 \%$ on admission to $26.2 \%$, range $22-31 \%$ on the day before surgery. Two retrospective studies ${ }^{177} 178$ of a total of 75 children with DMD undergoing scoliosis surgery report no difference in duration of intubation (all $<24 \mathrm{~h}$ ) or total days in hospital after surgery in the 33 children who had preoperative vital capacity of $<30 \%$ predicted compared with those with vital capacity $>30 \%$. Routine postoperative use of NIV was considered vital to facilitate successful early extubation. ${ }^{178}$ Gill and colleagues ${ }^{179}$ report successful outcomes for scoliosis surgery on eight children (mean age 12 years) with a variety of neuromuscular disorders, who had a mean preoperative vital capacity of $20 \%$ and all of whom were established on NIV for nocturnal hypoventilation. All patients were extubated direct to NIV without complication. 
Predicting respiratory risk in children unable to do lung function

There is only one study which addresses preoperative assessment in children with NMW who are unable to perform lung function manoeuvres. ${ }^{180}$ In this retrospective study 110 children (10.8 \pm 4.9 years), including 62 with neuromuscular disease, undergoing scoliosis surgery had preoperative PSG-73 children had daytime (nap) PSG and 39 had overnight PSG. None of the PSG parameters predicted risk of prolonged ventilation. Nap PSG does not usually include REM sleep, and so may underestimate hypoventilation and thereby miss parameters that may be useful in predicting postoperative risk.

\section{Peak cough flow}

The evidence for peak cough flow as a predictor of difficulty with extubation is based on adult studies and is considered in the section on identifying children at risk of respiratory complications.

\section{Evidence statements}

Children with NMW who have preoperative vital capacity of $<60 \%$ predicted have increased risk of prolonged postoperative ventilation (evidence level 3).

Use of NIV in the immediate postoperative period facilitates early extubation and reduces respiratory complications (evidence level 3).

Surgery can be successfully carried out with appropriate perioperative care without increased respiratory complications in children with severe NMW and vital capacity $<30 \%$ predicted (evidence level 3).

\section{Recommendations}

- Surgery in children with NMW should take place in units with experienced paediatric surgeons, anaesthetists and physiotherapists, and where there are facilities for paediatric intensive care and NIV. [D]

- Extubation protocols for high-risk children (those with vital capacity $<60 \%$ predicted, and/or ineffective cough, and/or who already use NIV for hypoventilation) should include effective airway clearance techniques and immediate use of NIV following extubation. [D]

\section{Good practice point}

- Children with NMW who require surgery (including scoliosis surgery) should be assessed by a multidisciplinary team prior to any intervention. $[\sqrt{ }]$

\section{SCOLIOSIS}

The pathophysiology of scoliosis and its impact on lung function have been discussed in the section on respiratory complications of NMW. The clinical effect of scoliosis is not limited to changes in lung function. Scoliosis distorts the centre of gravity, and necessitates compensatory pelvic or neck distortion. This affects posture and consequently pressure areas, and in patients who are wheelchair bound, this can lead to difficulties with positioning and discomfort. Interventions in children with scoliosis include bracing and surgery.

Traditionally braces have been made of rigid light plastic material and are usually fitted from the axillae to the iliac crests. More recently, non-rigid dynamic braces have been developed which appear to be more comfortable and better tolerated than rigid braces but no comparative studies of their effectiveness at improving posture or controlling spinal curvature are available. Vital capacity and tidal volume are reduced when wearing a brace as a result of restricted chest and abdominal expansion, and this must be taken into account when using a brace in a child with significant respiratory weakness, particularly if they have been asked to wear the brace when sleeping, when they will be most at risk of hypoventilation. Bracing may be useful in improving sitting posture. The benefits in terms of preventing progression of scoliosis are less certain. Wearing a brace can be uncomfortable and poorly tolerated, and not possible at all in some boys with DMD because of obesity.

Scoliosis surgery improves sitting comfort, appearance and QOL. It does not lead to an improvement in vital capacity, and whether it changes the rate of decline in respiratory function is uncertain. The optimal timing of scoliosis surgery in children with NMW is not clearly defined, and varies in different centres. The decision to operate is influenced by a number of factors which include curve progression, sitting comfort in wheelchairbound patients, mobility and pain. Early surgery with a flexible and relatively mild curves (Cobb angle of $45-60^{\circ}$ ) and good lung function (vital capacity over $50 \%$ predicted) is less demanding to the patient and surgeon. Most conventional surgical techniques involve manipulating the spine into a favourable position and fixing it in that position. This stabilises the spine and prevents further progression of the curve but also arrests growth in the fused area. Posterior fusion performed in young children (under 10 years of age) may result in a torsional (crankshaft) deformity as the anterior portion of the spine continues to grow. Growing systems with expandable rods which lengthen as the spine grows and allow optimisation of growth potential are more recent developments which may be useful if scoliosis surgery is contemplated in very young children. These systems incorporate single or dual rod technology to correct spinal curvature. A desire to allow maximum growth means that surgery is often delayed until the child is at least 10 years of age. While $80 \%$ of thoracic height is achieved by 10 years of age, thoracic volume is only $50 \%$ of adult values, ${ }^{181}$ and the impact of spinal fusion at this age on final thoracic volume is not known. Good surgical correction for Cobb angles of $>60^{\circ}$ is possible, provided that the spine retains some flexibility. If the spine has become rigid, spinal fixation can prevent any further progression of the scoliosis. Spinal surgery in children with NMW is associated with a greater morbidity, mortality and length of stay than in most other children with scoliosis. With appropriate planning and postoperative care, scoliosis surgery can be performed in children with vital capacity below $20 \%$ predicted, including children already using NIV prior to surgery, without excess respiratory morbidity (see section on planning for surgery).

\section{Evidence}

\section{Effect of bracing on lung function}

The evidence that wearing a brace reduces lung function comes from four observational studies of a total of 47 children with NMW and scoliosis, caused by a variety of diseases (predominantly DMD and SMA). Three studies showed a mean reduction in vital capacity of approximately $20 \%$ (range $4-37 \%$ ) when wearing a spinal brace. ${ }^{182-184}$ One study of eight children with severe SMA found that wearing a brace decreased tidal volume, increased respiratory rate and reduced respiratory system compliance. $^{185}$

\section{Effect of bracing on progression of scoliosis}

The evidence for the effect of bracing on progression of scoliosis comes from four uncontrolled observational studies of children with DMD and SMA. ${ }^{20} 186-188$ Evans and colleagues ${ }^{187}$ report a retrospective series of 54 children with SMA types 1-3, 43 of whom developed scoliosis. Bracing failed to control progression of scoliosis in children with severe weakness (types 1 and 2) but appeared to control progression in give children with less severe 
disease (SMA type 3). Cambridge and Drennan ${ }^{188}$ report that in 32 boys with DMD, spinal orthoses slowed the progression of scoliosis from an average of $16^{\circ}$ per year to around $10^{\circ}$ per year, but did not affect the final severity of scoliosis (average severity Cobb angle of $75^{\circ}$ ) in 30 out of the 32 subjects. Brooke and colleagues $^{20}$ report a retrospective review of 283 boys with $\mathrm{DMD}$ and 10 with BMD over a 10 -year period. Eighty-nine of 120 patents (75\%) over 11 years of age developed a scoliosis, 43 of whom had Cobb angles of $>30^{\circ}$. The use of thoraco-lumbar braces did not appear to affect the progression of scoliosis and there was a positive correlation between increasingly severe scoliosis and the duration of brace use. The authors noted that compliance with brace wearing was poor. Seeger and colleagues $^{186}$ report that in 24 boys with $\mathrm{DMD}$, scoliosis progressed from a mean value of approximately $10^{\circ}$ for children under 12 years to a mean of $75^{\circ}$ by the age of $16-18$ years and noted that the scoliosis severity in boys using modular seats, spinal jackets (worn $7 \mathrm{~h}$ per day) and custom-moulded seats were not significantly different from those observed using unmodified wheelchair seats. Colbert and Craig (1987) ${ }^{189}$ report a prospective study of 22 boys with $\mathrm{DMD}, 7$ who wore a brace (for $7 \mathrm{~h}$ /day, 6 days/week) and 15 who did not (either refused or stopped wearing the brace within 3 months). Over an observation period ranging from 2 to 7 years, the wearers showed a slightly slower rate of scoliosis progression, but with no difference in final severity.

\section{Evidence statements}

Wearing a rigid spinal bracing causes a reduction in both tidal ventilation and vital capacity in children with neuromuscular disease (evidence level 3).

In boys with $\mathrm{DMD}$, bracing may slow the progression of scoliosis, but does not affect final scoliosis severity (evidence level 3).

\section{Effect of scoliosis surgery on lung function}

The evidence of the effect of scoliosis on vital capacity is based on 13 studies of a total of 481 children with NMW, almost all with DMD or SMA. Three studies ${ }^{190-192}$ compared the rate of decline of vital capacity before and after surgery in the same individuals; five studies ${ }^{172}{ }^{193-196}$ compared the rate of decline to a non-operated group comprised either of children who did not yet need surgery, children in whom surgery was considered too high risk, or children who refused surgery; and five studies ${ }^{26} 179{ }^{197-199}$ compared a single preoperative mean vital capacity to one taken 2 or more years after surgery. Eight of the studies ${ }^{26} 172179190192194198199$ indicate that the rate of decline of vital capacity was reduced after surgery, or that vital capacity was maintained close to preoperative levels. Five studies $^{191} 193$ 195-197 found that the rate of decline of lung function was unaffected by surgery or was the same as that in a control group.

All the studies are retrospective. When there are control groups they are not matched. Without comparison to an appropriate control group it is hard to interpret changes in rate of decline of vital capacity, which are nonlinear and may plateau in late teenage years.

\section{Recommendation}

- The effect of wearing a spinal brace on respiratory function should be assessed and weighed against the limited evidence of benefit in terms of affecting final scoliosis severity. [D]

\section{Good practice point}

- The primary consideration when planning surgery for children with scoliosis associated with NMW should be comfort and QOL. [ل]

\section{OTHER PROBLEMS AND INTERVENTIONS THAT IMPACT ON RESPIRATORY HEALTH}

\section{Excessive oral secretions}

Drooling (sialorrhoea) can be problematic in children with inefficient tongue control or significant bulbar dysfunction. Dental malocclusion, poor oral health and nasal obstruction from enlarged adenoids can also lead to difficulties coping with oral secretions. Simple measures such as postural adjustment may help to alleviate the problem. Salivary secretion is controlled by the autonomic nervous system and as a consequence, anticholinergic medications have been used to regulate production of saliva. Saliva is important in the preparation of food boluses for swallowing, and medication should be used with caution; swallowing problems can be exacerbated if the mouth is dry or oral secretions become more viscous. Glycopyrrolate is frequently used, but dosage needs to be balanced by any side effects, commonly constipation. Hyoscine patches, with their gradual transdermal absorption, are an alternative to oral medication, although there can be variable results in children, in view of the difficulties with dosage titration and incidence of allergic skin reaction. The use of botulinum toxin as an injection into the salivary glands has become an option for children with nonprogressive neurological disorders such as cerebral palsy, where excessive sialorrhoea is particularly problematic and not well controlled by oral medications. The use of botulinum toxin has not been reported in children with neuromuscular conditions.

\section{Feeding and swallowing difficulties}

Difficulty with chewing and swallowing food are common in children with NMW, reflecting weakness of muscles in the cheeks, lips, tongue and pharynx, and frequently contribute to undernutrition. Problems with feeding and swallowing may be present from early life, for example, in children with severe forms of SMA, or may appear much later in the disease course, such as in boys with $\mathrm{DMD}$. Although oral and pharyngeal weakness can increase the risk of aspiration lung disease, aspiration is a relatively rare event and an uncommon cause of respiratory exacerbation (see section on studies to identify aspiration lung disease).

\section{Gastro-oesophageal reflux}

Gastro-oesophageal reflux is common in children with neuromuscular disorders, particularly in non-mobile children. Antacids, histamine $\mathrm{H} 2$-receptor antagonist, proton pump inhibitors and pro-kinetic agents may relieve symptoms, although there is concern that prolonged use of gastric acid suppression may be associated with an increased risk of gastroenteritis and community-acquired pneumonia. ${ }^{80}$ Antireflux surgery may be beneficial for gastro-oesophageal reflux disease refractory to medical management. In some cases, gastro-oesophageal reflux may be exacerbated by a gastrostomy procedure, ${ }^{200}$ although studies of children with neurological impairment suggest differently. ${ }^{201} 202$ Children with severe bulbar dysfunction and muscular weakness, such as those with SMA type 1, have been considered to be poor candidates for an anti-reflux procedure because of the high perioperative risk and overall poor prognosis ${ }^{203}$; centres advocating for a fundoplication procedure and gastrostomy insertion undertaken together shortly after diagnosis, in conjunction with a rigorous postextubation and airway clearance strategy, have reported that this can be accomplished safely in these children. ${ }^{200}$

\section{Gastrostomy feeding}

Feeding via gastrostomy tube placement has become increasingly important in maintaining the nutritional status of children 
with a variety of long-term conditions. Gastrostomies can be inserted via percutaneous methods under endoscopic guidance or via open or laparoscopic surgical techniques. In some adult neuromuscular patients with little or no autonomous breathing ability, gastrostomies have been performed avoiding intubation and general anaesthesia. ${ }^{204}$ Percutaneous image-guided gastrostomy and gastro-jejunostomy tube insertion under local anaesthetic and sedation have also been described in children with type 1 SMA. ${ }^{205}$ The timing of gastrostomy insertion varies between different centres, with some centres preferring the proactive approach, and others feeling that risks of surgery, however small, should be avoided in asymptomatic individuals. $^{80}$ In infants with very severe weakness and limited life expectancy such as the severest form of SMA type 1, careful consideration of the benefits of invasive procedures resulting in the medicalisation of tasks such as feeding, need to be balanced against the quality of the remaining months of life. Aside from the anaesthetic risk associated with poor lung function and ineffective airway clearance, perhaps coupled with the positional problems caused by scoliosis, gastrostomy insertion is associated with several complications, most often confined to the local gastrostomy site, including infection, bleeding, granuloma formation, and dislodged gastrostomy tubes.

\section{Respiratory tract infection}

Respiratory tract infections in children with neuromuscular disorders can significantly reduce respiratory muscle strength, resulting in symptoms of shortness of breath, reduction in vital capacity and acute hypercapnia. Respiratory syncytial virus (RSV) infection predominates in young children, while rhinovirus, influenza and parainfluenza virus infections are common in older children and adults. Although it is unclear how infection results in reduced muscle strength, acute viral infection can be associated with direct muscle injury and sepsis-induced respiratory muscle dysfunction can be mediated by oxygen-derived free radicals. Abnormalities in neuromuscular transmission have also been demonstrated during acute phase of viral illness. The risk of serious pulmonary complications resulting from RSV infection led the American Academy of Pediatrics to recognise neuromuscular disease in its revised guidelines for RSV immunoprophylaxis. Multicentre prospective data collection of children receiving RSV prophylaxis identified that $1.8 \%$ of children with central nervous system or neuromuscular disorders required hospitalisation for an RSV illness compared with $1 \%$ of the remaining children considered at high risk. ${ }^{206}$ Clinical management plans for children with NMW often include early or prophylactic use of antibiotics for respiratory exacerbations, although there are no studies which show that this approach is beneficial.

\section{Evidence}

\section{Use of medications to control oral secretions}

There is no evidence for the use of anticholinergic medication to control oral secretions in children with NMW.

\section{Feeding and swallowing difficulties}

Evidence for feeding and swallowing difficulties occurring in children with NMW has been drawn from one case-control study in children with $\mathrm{DMD}^{207}$ and six observational studies in children, two of which involved analysis of questionnaires 208209 and four studies which reported findings of clinical assessment, videofluoroscopy and $\mathrm{pH}$ studies in patients with a variety of neuromuscular conditions. ${ }^{81-84}$ Jaffe and colleagues ${ }^{207}$ compared responses to a questionnaire on symptoms of upper gastrointestinal dysfunction of 55 children with $\mathrm{DMD}$ from a regional neuromuscular clinic, aged matched to 55 healthy controls. Symptoms of dysphagia were significantly more common in children with DMD than in controls. Willig and colleagues ${ }^{209}$ received 409 responses to a questionnaire on alimentation problems experienced by seven groups of patients with neuromuscular conditions. A total of $36.5 \%$ of patients with SMA reported at least one problem with swallowing, predominantly within the buccal phase, whereas $53 \%$ of respondents in the DMD group (18.7 \pm 5.7 years) reported problems with mastication. In a population of 118 patients with $\mathrm{DMD}$ divided into age cohorts, correlated with levels of motor difficulty and use of ventilatory support, Pane and colleagues ${ }^{208}$ reported difficulties with mouth opening, chewing difficulties and choking (21/118), with 31 patients modifying their diet accordingly. Eight per cent of patients underwent gastrostomy insertion at a mean age of 23.6 years, all within 1 year of tracheotomy and all still regularly having food by mouth. Poor weight gain became an increasing problem in adolescence and adulthood. Although choking was one of the most feared, it occurred relatively infrequently, and was most prominent in the ventilated group. A retrospective case note review ${ }^{83}$ of 19 children with nemaline myopathy (18 ambulant) found 17 children had a history of feeding difficulties. Seven out of 10 children had abnormalities on videofluoroscopy. Significant residue was noted throughout the pharynx in all cases, increasing the risk of late aspiration. Eleven out of 19 $(61 \%)$ children had recurrent respiratory infections in the first 3 years of life. An observational study ${ }^{81}$ reported abnormal feeding assessment findings, confirmed by videofluoroscopy, in 13 of 14 children, aged 2-14 years with congenital muscular dystrophy. Two studies ${ }^{82} 84$ described feeding problems in patients with $\mathrm{DMD}$, where videofluoroscopy demonstrated food residue in the pharynx and around the laryngeal inlet. Although a Cochrane review failed to determine the benefit of dietary or feeding advice, ${ }^{210}$ in clinical practice, it seems sensible to continue to adopt strategies where the patients derive benefit.

\section{Evidence statements}

Feeding and swallowing difficulties are common in children with neuromuscular disease and are frequently under-recognised (evidence level 2-).

Oral and pharyngeal dysfunction increases with advancing age in boys with DMD (evidence level 3).

\section{Good practice points}

- Videofluoroscopy and feeding advice from a specialist therapist is indicated in children with a history of recurrent chest infection or swallowing difficulties. [ $\sqrt{ }]$

- A problem-orientated approach to nutrition should aim to minimise risk of aspiration, optimise nutritional status, promote comfort, and balance the positive social consequences of continued oral feeding. $[\mathrm{J}]$

\section{Diagnosis and treatment of gastro-oesophageal reflux}

One case-control study ${ }^{207}$ and two observation studies ${ }^{81} 208$ describe findings of gastro-oesophageal reflux in children with $\mathrm{DMD}$ and congenital muscular dystrophy. In the case-control study, ${ }^{207}$ DMD patients were significantly more likely to complain of dysphagia, choking while eating, heartburn and vomiting during or after meals than age-matched normal control children. In a comparison between ambulatory $(n=22)$ and nonambulatory subjects $(n=33)$ with $\mathrm{DMD}$, only heartburn was found to be significantly more prevalent in the non-ambulatory group ( $\mathrm{p}<0.02)$. Although the majority of $118 \mathrm{DMD}$ patients in the study by Pane and colleagues ${ }^{208}$ complained of occasional episodes of heartburn, gastro-oesophageal reflux requiring pharmacological treatment was only reported in five patients 
(4\%) all aged over 13 years. Philpot and colleagues ${ }^{81}$ describe clinical evaluation and videofluoroscopy in 14 children with merosin-deficient congenital muscular dystrophy. Only the youngest child, aged 2 years, had a normal study. Oesophageal $\mathrm{pH}$ studies were performed in 11 of 14 children who gave a history of chest infections, choking, vomiting or abdominal pain. In the eight children who tolerated the procedure, oesophageal reflux was demonstrated in six. These six children, all with a history of recurrent chest infection, also had abnormal videofluoroscopy with food penetrating the laryngeal inlet. No cough reflex was triggered in three children, indicating a risk of silent aspiration. Frequency of chest infections decreased after treatment. Laparoscopic fundoplication during gastrostomy placement is described in two studies of children with type 1 and severe type 2 SMA. ${ }^{200} 211$ A total of 17 children, 14 of whom had clinical evidence of gastro-oesophageal reflux, underwent a combined procedure, with extubation immediately in the postoperative period to NIV and aggressive airway clearance methods. Mean weight for length percentiles doubled in one of the studies ${ }^{200}$ and the number of respiratory-related hospitalisations decreased in both studies over the subsequent 12 months, though small numbers in both studies meant that statistical significance was not reached.

\section{Evidence statements}

Gastro-oesophageal reflux is common in children with neuromuscular disease and may contribute to aspiration lung disease (evidence level 2-).

Laparoscopic fundoplication during gastrostomy placement in children with type 1 and severe type 2 SMA can be undertaken safely and is effective in reducing gastro-oesophageal reflux. It may diminish respiratory-related hospital admissions (evidence level 3).

\section{Gastrostomy feeding}

Six observational studies describe gastrostomy insertion in patients with a range of neuromuscular conditions. Two retrospective case note reviews ${ }^{212} 213$ identified a total of 45 patients with a range of neuromuscular conditions in whom feeding via gastrostomy improved the weight for age ratio during the 1-year follow-up period. In one of the studies, the number of documented chest infections diminished after gastrostomy insertion. ${ }^{212}$ In the other study, local complications at the gastrostomy site were reported in 6 of 12 patients, all of which resolved swiftly. Three of five patients who had presented with previous gastro-oesophageal reflux had persistent problems post procedure, and despite medication, all developed at least one episode of pneumonia. ${ }^{212}$ Martigne and colleagues ${ }^{214}$ described an increase in the body weight for age ratio in 25 patients with DMD aged 11-38 years. Nutritional recovery was considered slow or incomplete as only 9 of 25 patients attained or exceeded their median value for weight/age ratio 1 year post gastrostomy placement. The complication rate in this study was also high (84\%) with 11 of 21 patients suffering complications within the first month, comprising a pneumo-peritoneum (3), abdominal parietal abscess (2), pneumonia due to presumed food aspiration (1) and local infection, general abdominal pain, nausea and vomiting. Fourteen patients subsequently had complications after the first month resulting in problems around the stoma (10), tube migration (2), and accidental extrusion (1). Ten patients had pneumonia after gastrostomy placement but due to the retrospective nature of the study, it was difficult to ascertain whether the episodes were due to aspiration. Durkin and colleagues ${ }^{200}$ describe the course of laparoscopic fundoplication and gastrostomy in 12 infants with SMA type 1, in whom postoperative respiratory management and discharge criteria were standardised. All patients were extubated immediately postoperatively, with no significant complications. The average time to full feeding and length of hospital stay were $42 \pm 4.9 \mathrm{~h}$ and $78 \pm 22.5 \mathrm{~h}$ respectively. Mean weight for length percentile was doubled at a year postoperatively $(p=0.03)$. Sy and colleagues $^{205}$ identified 12 children with SMA type 1 who had percutaneous gastrostomy or gastrojejunostomy insertion at a median age of 6.1 months (range 2.2-15.8 months); four children tolerating the procedure under local anaesthetic, and six children with sedation. General anaesthetic was required for two children, both of whom were already intubated. Seven of 12 children had no major complications in the first 30 days, although the tube was changed to a gastrojejunostomy tube because of gastro-oesophageal reflux disease in two children. The 30 -day mortality rate was $16.7 \%$. Aspiration pneumonia occurred in 5 of 12 children (41.6\%) within 30 days of their procedure, although four children had a history of pneumonia prior to tube placement. Bach and colleagues ${ }^{204}$ describe the procedure of open gastrostomy for ventilator-assisted or supported patients avoiding tracheostomy, intubation or general anaesthesia. The procedure was performed in 62 adult NIV users, with amyotrophic lateral sclerosis $(n=44)$, muscular dystrophy $(n=10)$ and other conditions $(n=8)$, using local anaesthetic and intravenous sedation. There were no complications and all patients subsequently gained weight. Mean post-gastrostomy survival was 38.8 months ( \pm 6.2 months).

\section{Evidence statement}

Gastrostomy feeding can improve and maintain adequate nutrition in children with NMW (evidence level 3).

\section{Respiratory tract infection}

The negative impact of respiratory infection in patients with neuromuscular disease is demonstrated by one prospective study of a mixed population of 25 adults and children aged between 7 and 40 years (mean age 20 years) with varying neuromuscular conditions, but stable respiratory status. ${ }^{70}$ Follow-up was continued for a month following complete resolution of symptoms of the respiratory tract infection. Significant reductions of up to $50 \%$ were noted in vital capacity and maximal inspiratory and expiratory pressures (mean reductions $50.1 \%, 51.5 \%$ and $26.8 \%$ respectively) in the 10 subjects who developed respiratory tract infections. Respiratory parameters remained reduced in two patients and significant episodes of hypercapnia continued in four patients, post infection. There is evidence that severe RSV infection in infants with neuromuscular disorders leads to considerable morbidity and higher mortality. A prospective cohort study ${ }^{215}$ covering six consecutive seasons evaluated whether children hospitalised with RSV infection with a clinically relevant NMW were at risk of complications. In total, 1568 RSV infections were prospectively documented in 1541 children. Forty-one of $73(56 \%)$ children with NMW had at least one additional risk factor for a severe course of the infection. Median age at the time of severe RSV infection was higher in children with NMW (14 months vs 5 months) and a higher proportion of children in the NMW group had to be mechanically ventilated (9.6\% vs $1.9 \%)$. The attributable mortality was also significantly higher in the NMW group (5.5\% vs $0.2 \%$; $<<0.001$ for all). A cohort study over an 8-year period using intensive care unit admission as a marker of severity of RSV infection found that a considerable proportion of children had serious underlying conditions. $^{216}$ Of the $4.4 \%$ deaths directly related to RSV infection, all had pre-existing conditions, $15 \%$ with neuromuscular disease. Although annual influenza vaccination is 
frequently advocated for high-risk groups, the evidence for efficacy in these groups is sparse. One prospective study ${ }^{217}$ has investigated whether children with neurological or neuromuscular disease had an increased risk of respiratory failure if hospitalised with viral influenza. This study of communityacquired and laboratory-confirmed influenza, conducted over four seasons, showed that children with neurological $(n=81)$ or neuromuscular impairment $(n=8)$ were, on average, six times more likely to develop respiratory failure requiring ventilation, although removing the children with a diagnosis of 'recurrent febrile seizures' reduced the OR to just 1.5.

\section{TRANSITION TO ADULT CARE}

'Transitions occur throughout life and are faced by all young people as they progress from childhood through puberty and adolescence to adulthood, from immaturity to maturity and from dependence to independence' (Department of Health, 'Transition: Moving on well', http://www.dh.gov.uk/en/Publicationsandstatistics/Publications/PublicationsPolicyAndGuidance/DH_083592, March 2008). Transition relates to a change towards adult status in relation to the law, education, health and social services, and with respect to friends, employment and social status within the wider community. Transition to adulthood for young people with muscle disease involves navigating a vast range of subjects, services and professionals at a time of life which is already vulnerable for most, whether living with a disability or not. Whereas for most young people, transition is a process during which independence is gained through greater choice and increased autonomy, for a young person with a progressive muscular condition, it can be a period when skills are lost and dependence on others increases, such as loss of independent mobility or establishment of ventilator support.

Young people with disabilities and their families often find the transition to adulthood stressful and difficult. Policy initiatives around transition have received much attention in view of the current poor coordination of public services between child and adult care. ${ }^{218}$ The transition from children's to adult services for those with a neuromuscular condition has been identified as a time when it is crucial that families have access to support, information and planning. A UK survey of service provision for adults with chronic neuromuscular disorders ${ }^{219}$ highlights a number of areas of concern, not limited to the process of transition, but also with the availability of appropriate specialist adult services in some areas of the UK.

With increasing survival of children with conditions such as DMD well into adult years and the impact of technologies such as home mechanical ventilation, there is a growing need to bridge the divide between child and adult-orientated specialist services. As an example, the teenager with $\mathrm{DMD}$ is more likely to require increased respiratory monitoring and support during this period, making it essential that there are adult respiratory teams available with appropriate expertise to care for him. Although access to specialist clinical services (not specifically for neuromuscular disease), is generally good, funding for respiratory support is variable and joint paediatric/adult or adolescent clinics are sparse. ${ }^{219}$

The multidisciplinary setting of many community paediatric units is ideal to coordinate care, equipment and services in home and school settings alongside the expert advice from specialist centres. In many areas of the UK this coordination becomes fragmented as families have to negotiate appointments with a number of different adult specialists and different agencies and at a time when the financial and social burden of care escalates. The role of the family in facilitating transition also appears crucial. $^{218}$ Conversely, those young people without strong parent advocates may receive a lower standard of support during the transition process, ${ }^{220}$ thereby limiting medical care, social, financial and further educational opportunities. A key-worker can play a vital role in ensuring transitional needs are driven by the young person, alongside parents. ${ }^{218} 220$

\section{What problems can be expected?}

Muscle weakness will progress in some conditions, resulting in increased ventilator dependency and there may be worsening cardiomyopathy, for example, in young men with DMD. With increasing survival, disease processes that are common in the general population (such as diabetes mellitus and deep vein thrombosis), can arise, and present particular challenges for young adults with $\mathrm{NMW}^{221}$ Some complications are disease specific, such as autonomic dysfunction that can occur in older patients with SMA. Care plans must accommodate disease progression and increasing ventilator dependence, and teams should be aware of new additional health problems that are not always amenable to the usual treatment methods, such as those that require surgical intervention under general anaesthesia.

With advances in respiratory management, prolonged survival can start to impact upon the adolescents' perception of $\mathrm{QOL}$ particularly in the psychosocial domains, such as interpersonal relationships. ${ }^{221} 222$ Transition is a time when school, with its daily structure, activities and the companionship that it provides, also comes to an end. The burden of care upon families and society increases, as complex packages of care require funding and implementing, combined with the need for occupational, social and leisure activities.

Transition is also a time for serious decision-making around the risks and benefits of potential therapies with the adolescent actively involved in the process. Paediatricians need to be aware when discussing the future and possible treatment options with children and their parents that some literature on the prognostic implications of treatments is based on studies that were conducted at a time when survival was not expected or when prolonged follow-up of participants was not envisaged. ${ }^{221}$

\section{Different models of transition}

Several models of transition exist, although the most predominant and well established is that of 'condition-specific' transition, when paediatric providers work directly with adult providers to ensure prioritisation of the management of specific conditions. Joint working, education and research between adult and paediatric services are essential. The generic adolescent model in which clinics are coordinated by an adolescent specialist has many merits, with the recognition that young people prefer same-age groupings ${ }^{218}$; the disadvantage of this model is the requirement of a further intermediary team with whom the young person and carers need to develop a relationship. For relatively uncommon conditions, such as neuromuscular disorders, there is still likely to be a requirement for liaison with individual specialist services for many areas of the UK, as tertiary specialist care may be geographically remote. Some regions have developed an innovative care network to overcome some of the difficulties of widely dispersed populations, providing support to local teams. ${ }^{219}$ It has been suggested that there is a growing trend to reintroduce the general practitioner (GP) as the coordinator during the transition process. By providing a general overview and with local knowledge of services, the GP is able to refer back to adult specialist services as necessary. This primary transition model has met with opposition in view of the current divide between primary and specialist 
care and yet it is only the GP who provides lifelong continuity in healthcare, often beyond a generation. ${ }^{220}$

Whichever process is followed, a number of principles underpin success. Recurring themes are described-information, communication, planning and coordination. ${ }^{218} 223$ A project highlighting the views of young people with a neuromuscular condition about the transition process voiced similar opinions (Muscular Dystrophy Campaign publication. 'Becoming an adult: transition for young men with DMD', www.musculardystrophy.org, April 2010) and reported that young people:

- felt that care should be consistent and responsive to individual need, welcoming the concept of a health portfolio to encourage effective information sharing between different agencies;

- wished to be supported in directing their individual care needs and to have access to a wide range of information;

- felt that the process should occur gradually over a number of years with a timetable of transition outlined;

- thought that peer support programmes were helpful in coping with uncertainties of adolescence;

- preferred informal care strategies that are tailored to individual need.

The process of transition from paediatric to adult services for young people with neuromuscular conditions in the UK is not ideal and further studies are required to evaluate which combination of components works best. The variation in population density across the UK and the variability in local services mean that it is not likely that one transition model will fit all; nevertheless, a national approach to minimum standards of care would help to reduce the current inequalities that exist.

\section{Evidence}

Additional health problems occurring in young adults with NMW are illustrated in three observational studies. Birnkrant ${ }^{221}$ reports that of 19 young men with $\mathrm{DMD}, 18$ received assisted ventilation (16 NIV) of whom 12 received $24 \mathrm{~h}$ ventilatory support; 15 had undergone gastrostomy placement for poor nutrition and/or difficulties swallowing; 6 had nephrolithiasis; 2 had developed diabetes mellitus; 2 had a deep venous thrombosis; and 1 each had gallstones and inflammatory bowel disease. A survey of 65 Danish adults with DMD aged $18-42$ years $^{222}$ describes daily life positively, although the majority yearn for a loving relationship and few have the opportunity to undertake further educational or vocational opportunities. Pain is common, occurring in $40 \%$ of subjects on a daily basis, coupled with frequent fatigue. This evidence concurs with results from a study of 42 youths with neuromuscular disease ${ }^{224}$ in which $23(55 \%)$ were identified as having chronic pain, mainly in the legs and with $83 \%$ using pain medication.

Only one observational study specifically evaluated the current practices of transition for young people with neuromuscular disorders, from a district in the UK, comparing experiences with the current Department of Health guidelines. ${ }^{225}$ Responses to questionnaires were obtained from 21 young adults and 17 carers (54\% response rate). Two focus groups were held to obtain views, with a number of key themes emerging. Young people expressed the need for information resources and access to information on adult services, with identification of a key worker. They expressed a preference for transition to occur at aged 18 years, but that the current transition practice was too abrupt and should be a more gradual process.

Experience has shown that for practical reasons, transition at 18 years poses difficulties, as children's wards and paediatric intensive care units do not usually accept those over 16 years.
Therefore if an acute inpatient stay is required before transition commences, admission to an adult ward can result in a precipitous and unsatisfactory handover of care to adult teams. A survey of 32 neuromuscular clinics across 25 cities in the $\mathrm{UK}^{219}$ highlighted the variability in practice and availability of resources and services. Responses were received from 30 clinicians based in 24 locations, with the majority of clinics based in large regional centres. Only 11 clinics (42\%) requiring transfer of services to adult care had a service in place to do so, excluding clinics where the same team saw adults and children. A total of $32 \%$ of neuromuscular disease clinics did not routinely monitor respiratory function and $10 \%$ did not routinely perform ECG, suggesting variable pathways for referral. Domiciliary support was fully funded in $43 \%$ and on an individual basis in $50 \%$. There was inconsistent access to therapy and nursing services and long waits for wheelchair provision. Many clinicians had a poor awareness of local services.

\section{Evidence statements}

For young people with a neuromuscular disorder, particularly $\mathrm{DMD}$, the teenage years are a period when their condition can change considerably, and new health problems may emerge, thereby increasing the importance of a smooth handover from child-orientated to adult specialist services (evidence level 3).

There is considerable variability across the UK in current practice and availability of resources and services for people with neuromuscular disease (evidence level 3).

Young people with neuromuscular disease feel that components of a successful transition revolve around information, communication and planning over a number of years (evidence level 3).

\section{Good practice point}

- A key worker can act as a valuable advocate, a source of knowledge and provide support for young people with neuromuscular disease and their families during the period of transition from paediatric to adult services. $[\sqrt{ }]$

\section{OOL AND PALLIATIVE CARE}

Increased survival of children with NMW has led to unanticipated medical, psychosocial and ethical issues. Advancement in medical technologies supporting longer-term care has outstripped the available research and information into these new issues, which have a profound effect on patients, their families and society. Improving and maintaining the QOL of children and young people is the main goal of paediatric palliative care. There is a common misconception that palliative care can only be delivered in the final stages of an illness, towards, and at the end of life. Palliative care can be delivered at any point in the disease trajectory of a child or young person with a life-shortening neuromuscular disorder. Many aspects of palliative care support can be offered in parallel with active treatment, even if full escalation of intervention to intensive care is planned.

\section{Quality of life}

Despite the widespread use of QOL as a term based upon a theoretical concept, no agreed and precise definition exists. In healthcare research, QOL includes a range of components ('domains') that are measurable and related to health, disease, illness and medical interventions. Most healthcare providers agree that health-related quality of life (HROOL) a subdomain of $\mathrm{QOL}$, is a multidimensional concept related to the physical aspect of a condition, emotional state and the ability to interact with others and have fulfilment and meaning.

The plethora of instruments to measure HRQOL, sometimes with extensive questioning within different domains, is a challenge for the user and the reader of scientific reports. 
Instruments may be generic or disease specific. Historically, many research instruments used to measure HROOL considered effects on physical, psychological and social functioning, and measured functional ability rather than participation in day-today life and subjective life satisfaction. As physical impairment impacts on everyday activities, low HRQOL is an expected outcome of such tools and may be inadequate and misleading. Assessing QOL in children is also a challenge; self-report is the gold standard for HROOL, particularly as there are inconsistencies in level of agreement between parents or carers and the child. Instruments should be age specific and if at all possible based on self-report.

\section{Evidence}

The Paediatric Quality of Life Inventory (PedsOL) tool has established reliability and validity within the wider paediatric population, although it is a generic research tool. This QOL tool contains both age-related and parental report. Iannaccone and Hynan ${ }^{226}$ have validated the PedsOL for children with SMA and Bray and colleagues $^{227}$ have used the PedsOL (generic scale) in boys with DMD. Further data ${ }^{228}$ support the feasibility, reliability and validity of the tool in children with SMA. These studies demonstrate poor to moderate agreement between child and parent responses, suggesting information provided by proxy respondents is not equivalent to that reported by the affected child.

There is now significant evidence ${ }^{229-233}$ that physicians often underestimate quality of life compared with patients and parents or carers. Studies show that parental decision-making regarding treatment is directly related to perception of QOL. ${ }^{234} 235$ Understanding HROOL from the perspective of the patient is essential to facilitate shared decision-making with healthcare professionals and parents or carers.

In spite of the prevalent perception that patients with severe physical impairment or chronic disease experience lower QOL than non-disabled persons, recent studies targeting selfperception have shown that QOL in patients with a progressive neuromuscular condition is not correlated with physical impairment or the need for NIV. ${ }^{235-245}$

\section{Evidence statements}

Physicians often underestimate QOL compared with patients and parents or carers (evidence level 3).

Proxy parent or carer $\mathrm{OOL}$ reports are not equivalent to those reported by patients (evidence level 2-).

Despite severe physical disability, children and young people with neuromuscular disorders report an acceptable $\mathrm{QOL}$ (evidence level 2-).

\section{Recommendations}

- Evaluating the child and parent or carer using multidimensional HRQOL assessment tools should be the standard for routine assessment in clinical practice and future clinical trials. [D]

- International standardised disease-specific tools for children and young people with neuromuscular disorders should be used if available to evaluate clinical interventions and patientrelated outcome measures with respect to QOL. [D]

\section{Ethical use of interventions}

NIV may be used for symptom management in respiratory failure without the goal of extending life. Alleviating the unpleasant symptoms of dyspnoea and the consequences of carbon dioxide retention may improve QOL. Respiratory management options now permit prolonged survival for children with NMW, including infants with SMA type 1. Use of ventilatory support in infants with SMA type 1, whether invasive or non-invasive, is not widespread in the UK. Decisions regarding long-term ventilation involve a weighing of 'burden' and 'benefits' for each individual and by their very nature are value laden and cannot be made unilaterally. Perhaps a sound 'transparent' and ethical approach is to recognise that the healthcare providers' own beliefs and attitudes might be offered in conjunction with the proposed treatment plan options. This may enable factual information to be delivered in conjunction with the healthcare providers' own experiences and values in a specific context.

\section{Evidence}

Several studies ${ }^{233}$ 246-248 demonstrate significant worldwide variation in attitudes and practice of physicians regarding respiratory management of patients with SMA type 1. Withholding and withdrawing life-sustaining ventilator assistance in this condition is accepted as standard medical practice by many physicians. Others advocate full long-term ventilatory support.

Some studies 248249 have shown a paternalistic approach to 'rationing' the use of ventilatory support, particularly in $\mathrm{DMD}$, with concern that such support may 'prolong dying' and 'extend suffering' and poor QOL. More encouraging results ${ }^{250-252}$ have shown physicians offering choice regarding ventilatory support; however this is often reported as being during an episode of respiratory failure. Yates and colleagues ${ }^{253}$ reviewed data for 28 children with neuromuscular disease admitted to the intensive care units over 15 years and found that $66 \%$ of admissions (47/69) were unplanned; 11 of the 28 children admitted as an emergency for respiratory failure required ventilatory support after discharge from the intensive care unit and 6 of these continued on home support. One study of 13 infants with SMA type 1 describes the use of NIV and MI-E to support clearly defined, goal-orientated care. ${ }^{96}$ This approach facilitated extubation and home discharge with positive feedback from parents. Parents and carers have reported that they should be given the choice to decide for or against home mechanical ventilation. Stressors were related to poor support in the context of financial and practical assistance. ${ }^{235}$

\section{Evidence statement}

Respiratory failure is usually predictable. Paternalistic medical advice can result in either uninformed implementation or withholding of ventilatory treatments. Lack of informed choice and equity of service prevents planning and limits decision-making (evidence level 3).

\section{Recommendation}

- Assisting patients, parents and carers to make informed choices that are consistent with their own values and preferences requires physicians to engage patients and their parents and carers in a process of mutual participation in decision-making with full disclosure of all information in a sensitive and timely fashion. [D]

\section{Good practice point}

- Open discussion across the multidisciplinary team regarding the type and duration of specific interventions encourages transparency and shared decision-making. [ $\sqrt{ }]$

\section{Psychological and mental health issues}

There is a need for increased mental health support for children and young people with neuromuscular disease and their carers. There are reports of increased maladaptive coping strategies, behavioural problems and mental health issues within this population and their parents or carers.

\section{Evidence}

The acknowledgement of stress factors and need for wider support is borne out in studies that have surveyed families of 
patients with DMD. ${ }^{234} 254-257$ Mental health and behavioural issues are perceived to be important, particularly as boys grow older. Parents show increased levels of anxiety and depression and self-reported poor psychological adjustment, particularly single parents and those with older sons. Carers of boys with $\mathrm{DMD}$ using home ventilation rated sleep quality as poor and reported associated negative emotional symptoms and illness burden on family health and relationships. ${ }^{245}$ The functional level of the child is directly related to maternal stress and QOL. ${ }^{258}$ Parents report a sense of loss each time they see physical signs of deterioration. ${ }^{235}$ A small study by Hodges and $\mathrm{Dibb}^{259}$ regarding the use of $\mathrm{DMD}$ self-help support groups uncovered a wide range of social comparisons and coping dimensions with positive and negative effects.

\section{Evidence statement}

Living with a neuromuscular condition increases mental health issues and stress factors in affected children and parents or carers, although both groups exhibit considerable adaptive ability (evidence level 2-).

\section{Recommendation}

- Benefit and burden of all interventions must be considered with respect to impact on mental health of patients and their families. [D]

- Acknowledgement of stress factors and mental health issues, requirement for wider support through organisations, and practical and focused individual care are needed for patients and their parents or carers. [D]

\section{Symptom management and end-of-life care}

Children and young people with neuromuscular disorders live with a significant burden of physical symptoms. Prolonged survival is associated with increasing morbidity of respiratory and non-respiratory health-related issues. Dyspnoea, particularly, is an under-recognised and under-reported symptom with a neglected evidence base. Dyspnoea is a subjective feeling, which can respond to treatment directed at the cause. Low-dose opioids may be used to manage distressing dyspnoea even when active treatment is pursued. One-third of the standard analgesic opioid prescription is a safe and appropriate dose to start. With slow titration of opioids, respiratory depression is unlikely. Fentanyl is generally not recommended as it can increase muscle tension and so possibly exacerbate dyspnoea. ${ }^{260}$ In the terminal phase of respiratory failure, frequent reassessment of symptoms allows effective management with a combination of non-drug measures and careful titration of opioids and psychotropic agents.

The paucity of information regarding end-of-life care in children with NMW is striking. There is emerging expertise among paediatric palliative care healthcare professionals regarding the symptom management of children and young people with NMW. Unfortunately this is not reflected in the available literature. Terminal respiratory failure is often the mode of death in children with NMW. Retention of carbon dioxide can result in physiological sedation. Overzealous administration of oxygen should be avoided, as this may result in prolonging the period of hypoxic agitation. Non-drug management of dyspnoea includes managing secretions, use of a fan or open window, massage and visualisation for anxiety, and appropriate positioning of the child. Medical management involves the careful use of opioids and psychotropic medication, including benzodiazepines for agitation. Towards the end of life, families may want to remove NIV equipment from the child's face. This requires sensitive discussion and careful timing and prompt symptom management.
Evidence

General symptom management

Studies report a significant prevalence of ongoing medical complications as a consequence of chronic illness in children with NMW. ${ }^{221}{ }^{242}$ A national survey of hospice provision for those with NMW showed a significant number of boys with $\mathrm{DMD}$ required some form of medical intervention and symptom management during routine respite stays. ${ }^{261}$

\section{Dyspnoea}

Breathlessness is a distressing symptom that can arise with inter-current infection or aspiration, in conjunction with cardiac failure, with progressive neuromuscular deterioration, and in the final stages of disease. As discussed previously, symptoms of respiratory distress in infants with SMA type 1 can be effectively palliated with NIV. The importance of effective secretion clearance and feeding management is emphasised in this report. ${ }^{96}$

\section{Pain}

There is a significant correlation between increased pain and fatigue and lower levels of general health, vitality and social function. Chronic pain has been investigated by a number of studies $^{224} 239262263$ and was found to be common in neuromuscular conditions. Poor pain management is known to be negatively correlated with QOL and impacts on many aspects of care, including respiratory care.

\section{End-of-life care}

Little is known about place of care during the final illness. Of 531 children with neuromuscular disease known to UK hospices, only 7 out of 47 deaths had occurred in the hospices themselves over the previous year. ${ }^{261}$ However, $85 \%$ of families (of those 47 deaths) accessed bereavement services and some used the "cold room' facility, neither of which is available on the NHS. Over a 15 -year period, 10 out of 28 children with known neuromuscular disease and a previous admission to paediatric intensive care units had died, with only four deaths actually occurring in the intensive care unit. ${ }^{253}$

\section{Evidence statement}

Pain symptoms are under-recognised and under-reported in neuromuscular disorders (evidence level 3).

\section{Recommendations}

- Routine pain evaluation should be part of standard clinical assessment in all children and young people with neuromuscular disorders. [D]

- Attention to the wider physical impact of chronic illness through vigilant symptom management is required. [D]

\section{Good practice points}

- Dyspnoea is a subjective feeling, which can respond to nondrug measures and treatment directed at the cause. [ $\sqrt{ }]$

- Low-dose opioids may be used to manage distressing breathlessness even when active treatment is pursued. With slow titration of opioids, respiratory depression is extremely unlikely. [ $\sqrt{ }]$

- In the terminal phase of respiratory failure, symptoms must be reassessed frequently and can be effectively managed by a multimodal approach, including careful titration of opioids and psychotropic agents. $[\sqrt{ }]$

\section{Advance care planning}

Assisting patients, parents and carers to make informed choices requires the multidisciplinary team to engage patients and their parents or carers in a process of mutual decision-making. Timing discussions of this nature can be difficult. Often a series of discussions with a variety of key healthcare professionals, 
support groups and peers with the condition or parents with a child with the same condition will help to consolidate the shared decision-making process. Promoting choice, through continued assessment of need, improving the quality and range of information offered and open discussion at key points in the disease trajectory, supports this process. Empowering young people to make decisions regarding their future care wherever possible, and protecting those who lack the capacity to do so, must form the basis for the decision-making process in the older age groups. ${ }^{264} 265$

Advance care planning is a voluntary process of discussion and review to help anticipate how an individual's condition may affect them in the future. If a patient or their parent/guardian wish, advance care planning records choices about care, treatment, and/or an advance decision to refuse treatment in specific circumstances. An advanced care plan is neither a legal document nor a 'do not resuscitate' order. An advanced care plan is likely to change in parallel with the disease progression. In paediatric practice, an advance care plan often incorporates wishes for emergency care (sometimes referred to as an 'Emergency healthcare plan') and ensures written information regarding future clinical management is discussed and documented and available should an emergency arise. Perpetual offers of intervention on a background of continual demise may preclude the opportunity for the patient and their parents or carers to plan end-of-life care and a dignified, symptom-free death.

\section{Evidence}

Chatwin and colleagues ${ }^{96}$ describe using advance care plans in a series of infants with SMA type 1 to assist in anticipatory decision-making regarding escalation in ventilatory support. Erby and colleagues ${ }^{257}$ report that none of the 18 parents of boys with DMD (aged over 7 years) interviewed acknowledged having explicit conversations about advance care plans within their families or with their sons or physicians. Parents did not have a working knowledge of the concept of advance care planning and attributed the process to 'do not resuscitate' orders.

Wiener and colleagues ${ }^{266}$ discussed advance care plans with a group of 20 adolescents with life-shortening illness and found that $95 \%$ thought the document would be 'helpful' or 'very helpful' to themselves or others. No patients found talking about the issue stressful but were more interested in discussing how they wanted to be remembered rather than medical decision-making.

Unplanned admission to intensive care units may be a trigger point for discussion; Yates and colleagues ${ }^{253}$ found that 36\% (10 out of 28) children with neuromuscular disease had died following admission to intensive care units and $70 \%$ were in the severe functional group. Two case series ${ }^{252} 267$ report on positive experiences of children and young adults with DMD in the formulation of life plans, regardless of the decision made about long-term ventilator assistance. A case study of a boy with DMD by Penner and colleagues ${ }^{268}$ describes the ethics of disclosure and emotionally charged challenges surrounding advance care plans.

\section{Evidence statement}

Advance care plans and similar approaches may be useful for children and young adults with a progressive neuromuscular condition in helping to anticipate need and prevent crisis management and last minute decision-making (evidence level 3).

\section{Recommendations}

- Advance care planning should be an integral part of the active management of children and young people with neuromus- cular disorders. Advance care plans can be used as a vehicle for information exchange and considered decision-making. [D]

- Patients and families need to have ownership of the advance care plan and be educated as to its uses. [D]

\section{Good practice point}

- Advance care plans should be reviewed by the multidisciplinary team on a regular basis. $[\sqrt{ }]$

\section{Role of palliative care}

Paediatric palliative care principles are directly applicable to the context of paediatric neuromuscular disease because the focus is on 'survivorship' and long-term multidimensional support aimed at maintaining quality of life. In neuromuscular disease management, a framework has developed that integrates the multidimensional approach of palliative care with curative interventions; focus is on what can be offered, rather than what cannot be done. Active palliative care in this context anticipates crucial milestones, which may precipitate vulnerabilities and offers flexible re-evaluation of goals of care in line with prognosis.

Children's hospices in the UK have developed an important role with regard to children and young people with neuromuscular disease offering a wide range of supportive services unavailable elsewhere. Many expert review papers state the importance of integrating palliative care services into the mainstream care of children and young people with neuromuscular disease; more objective studies are needed to endorse the benefits of this approach.

\section{Evidence}

Older studies ${ }^{269} 270$ suggest that the palliative care model has much to offer individuals with progressive neuromuscular conditions and their families. Children and young people with neuromuscular disease, especially those with $\mathrm{DMD}$, form a large proportion of the cases cared for by the children's hospices in the UK. ${ }^{261}$ Most support is multidimensional. Planned stays allow access to peer support and social activities that are often curtailed or restricted in the wider community as a consequence of disability. Referrals peak for DMD in adolescence at a time when those with DMD are losing ambulation and when patients often have greater physical and emotional needs.

\section{Recommendation}

- Families need access to skilled experts for multidimensional coordinated palliative care support, providing regular review of their needs at various stages in their condition. [D]

\section{Good practice point}

- Generic palliative care skills should be cascaded to other professionals providing neuromuscular services. $[\sqrt{ }]$

\section{SPECIAL CONSIDERATIONS}

This short section deals with issues that do not fit easily elsewhere within the guideline.

\section{Resuscitation training}

\section{Good practice point}

- Carers and parents of children with severe NMW, including all those using NIV and all those with tracheostomy, should have basic life support training. $[\sqrt{ }]$

\section{Written management plans}

\section{Good practice point}

- Written plans for the management of acute exacerbations, which include details of effective airway clearance methods, 
and ventilator settings when appropriate, and contact details of relevant healthcare professionals are recommended. [ $\mathrm{V}]$

\section{Congenital myasthenic syndromes}

Congenital myasthenic syndromes differ from most other causes of NMW in that they often cause variable weakness which can change from relatively mild weakness in an ambulant child to respiratory failure over the course of a few hours, or more rarely several minutes, often precipitated by an otherwise trivial viral infection. Respiratory assessment when these children are well can be misleadingly reassuring. There is no evidence base to support respiratory management guidelines for children with congenital myasthenic syndromes. Guidance on the respiratory management of this condition has been proposed by a workshop meeting held in $2009 .{ }^{40}$ The key points of this guidance are that: - Every child with congenital myasthenic syndromes should have fast track access to their local paediatric service in the event of illness or in an emergency.

- Children at high risk of respiratory complications, either by genotype or because of a history of respiratory illness or bulbar insufficiency, should be referred to their regional paediatric respiratory centre for assessment, including formal sleep polygraphy and to formulate a respiratory care plan.

- Carers of all children with congenital myasthenic syndromes should be provided with resuscitation training, including the management of choking. For children at high risk of sudden respiratory failure, training should include use of bag-valve-mask equipment.

- Children who have had multiple episodes of respiratory failure may require home non-invasive ventilation for emergency use.

\section{Competing interests None}

Provenance and peer review Not commissioned; internally peer reviewed.

\section{REFERENCES}

1. Howard RS, Wiles CM, Hirsch NP, et al. Respiratory involvement in primary muscle disorders: assessment and management. O J Med 1993;86:175-89.

2. Perrin C, Unterborn JN, Ambrosio CD, et al. Pulmonary complications of chronic neuromuscular diseases and their management. Muscle Nerve 2004:29:5-27.

3. Kurz LT, Mubarak SJ, Schultz P, et al. Correlation of scoliosis and pulmonary function in Duchenne muscular dystrophy. J Pediatr Orthopedics 1983;3:347-53.

4. De Troyer A, Deisser P. The effects of intermittent positive pressure breathing on patients with respiratory muscle weakness. Am Rev Respir Dis 1981:124:132-7.

5. Gibson GJ, Pride NB, Davis JN, et al. Pulmonary mechanics in patients with respiratory muscle weakness. Am Rev Respir Dis 1977;115:389-95.

6. McCool FD, Mayewski RF, Shayne DS, et al. Intermittent positive pressure breathing in patients with respiratory muscle weakness. Alterations in total respiratory system compliance. Chest 1986;90:546-52.

7. Estenne M, Heilporn A, Delhez $L$, et al. Chest wall stiffness in patients with chronic respiratory muscle weakness. Am Rev Respir Dis 1983;128:1002-7.

8. Misuri G, Lanini B, Gigliotti F, et al. Mechanism of $\mathrm{CO}(2)$ retention in patients with neuromuscular disease. Chest 2000;117:447-53.

9. Jeffery PK. The origins of secretions in the lower respiratory tract. Eur $J$ Respir Dis Suppl 1987;153:34-42.

10. Leith DE. The development of cough. Am Rev Respir Dis 1985;131:S39-42.

11. Yanagihara N, Von Leden H, Werner-Kukuk E. The physical parameters of cough: the larynx in a normal single cough. Acta Otolaryngol 1966;61:495-510.

12. Szeinberg A, Tabachnik E, Rashed N, et al. Cough capacity in patients with muscular dystrophy. Chest 1988;94:1232-5.

13. Ringqvist I, Ringqvist T. Respiratory mechanics in untreated myasthenia gravis with special reference to the respiratory forces. Acta Med Scand 1971; 190:499-508

14. McCrory MA, Kim HR, Wright NC, et al. Energy expenditure, physical activity, and body composition of ambulatory adults with hereditary neuromuscular disease. Am J Clin Nutr 1998:67:1162-9.

15. Willig TN, Carlier L, Legrand M, et al. Nutritional assessment in Duchenne muscular dystrophy. Dev Med Child Neurol 1993;35:1074-82.

16. Sproule DM, Montes J, Dunaway S, et al. Adiposity is increased among highfunctioning, non-ambulatory patients with spinal muscular atrophy. Neuromuscular Disord 2010;20:448-52.
17. Leroy-Willig A, Willig TN, Henry-Feugeas MC, et al. Body composition determined with MR in patients with Duchenne muscular dystrophy, spinal muscular atrophy, and normal subjects. Magn Reson Imaging 1997;15:737-44.

18. McDonald CM, Abresch RT, Carter GT, et al. Profiles of neuromuscular diseases. Duchenne muscular dystrophy. Am J Phys Med Rehabil 1995;74(5 Suppl):S70-92.

19. Goldstein M, Meyer S, Freund HR. Effects of overfeeding in children with muscle dystrophies. JPEN J Parenteral Enteral Nutr 1989;13:603-7.

20. Brooke MH, Fenichel GM, Griggs RC, et al. Duchenne muscular dystrophy: patterns of clinical progression and effects of supportive therapy. Neurology 1989:39:475-81.

21. Oda T, Shimizu N, Yonenobu K, et al. Longitudinal study of spinal deformity in Duchenne muscular dystrophy. J Pediatr Orthop 1993;13:478-88.

22. Aprin H, Bowen JR, MacEwen GD, et al. Spine fusion in patients with spinal muscular atrophy. J Bone Joint Surg Am 1982;64:1179-87.

23. Kilmer DD, Abresch RT, McCrory MA, et al. Facioscapulohumeral muscular dystrophy. Am J Phys Med Rehabil 1995;74(5 Suppl):S131-S9.

24. McDonald CM, Abresch RT, Carter GT, et al. Profiles of neuromuscular diseases. Becker's muscular dystrophy. Am J Phys Med Rehabil 1995:74(5 Suppl):S93-103.

25. Inal-Ince D, Savci S, Arikan H, et al. Effects of scoliosis on respiratory muscle strength in patients with neuromuscular disorders. Spine J 2009;9:981-6.

26. Robinson D, Galasko CS, Delaney C, et al. Scoliosis and lung function in spinal muscular atrophy. Eur Spine J 1995;4:268-73.

27. Yamashita T, Kanaya K, Yokogushi K, et al. Correlation between progression of spinal deformity and pulmonary function in Duchenne muscular dystrophy. J Pediatr Orthop 2001;21:113-16.

28. Galasko CS, Williamson JB, Delaney CM. Lung function in Duchenne muscular dystrophy. Eur Spine J 1995;4:263-7.

29. Dhand UK, Dhand R. Sleep disorders in neuromuscular diseases. Curr Opin Pulm Med 2006:12:402-8.

30. Katz SL. Assessment of sleep-disordered breathing in pediatric neuromuscular diseases. Pediatrics 2009;123:S222-5

31. Carroll JL, McColley SA, Marcus CL, et al. Inability of clinical history to distinguish primary snoring from obstructive sleep apnea syndrome in children. Chest 1995:108:610-18.

32. Suresh S, Wales P, Dakin C, et al. Sleep-related breathing disorder in Duchenne muscular dystrophy: disease spectrum in the paediatric population. $J$ Paediatr Child Health 2005:41:500-3.

33. Khan Y, Heckmatt JZ. Obstructive apnoeas in Duchenne muscular dystrophy Thorax 1994;49:157-61.

34. Quera Salva MA, Blumen M, Jacquette A, et al. Sleep disorders in childhoodonset myotonic dystrophy type 1. Neuromuscul Disord 2006;16:564-70.

35. Martinez-Rodriguez JE, Lin L, Iranzo A, et al. Decreased hypocretin-1 (Orexin-A) levels in the cerebrospinal fluid of patients with myotonic dystrophy and excessive daytime sleepiness. Sleep 2003;26:287-90.

36. Vianello A, Bevilacqua M, Salvador V, et al. Long-term nasal intermittent positive pressure ventilation in advanced Duchenne's muscular dystrophy. Chest 1994;105:445-8.

37. Baydur A. Respiratory muscle strength and control of ventilation in patients with neuromuscular disease. Chest 1991;:99:330-8.

38. Gigliotti F, Pizzi A, Duranti R, et al. Control of breathing in patients with limb girdle dystrophy: a controlled study. Thorax 1995:50:962-8.

39. Tzeng AC, Bach JR. Prevention of pulmonary morbidity for patients with neuromuscular disease. Chest 2000;118:1390-6.

40. Robb SA, Muntoni F, Simonds AK. Respiratory management of congenital myasthenic syndromes in childhood: Workshop 8th December 2009, UCL Institute of Neurology, London, UK. Neuromuscul Disord 2010;20:833-8.

41. Racca F, Del Sorbo L, Mongini T, et al. Respiratory management of acute respiratory failure in neuromuscular diseases. Minerva Anestesiol 2010;76:51-62.

42. Brunherotti MA, Sobreira C, Rodrigues-Junior AL, et al. Correlations of Egen Klassifikation and Barthel Index scores with pulmonary function parameters in Duchenne muscular dystrophy. Heart Lung 2007;36:132-9.

43. Katz SL, Gaboury I, Keilty K et al. Nocturnal hypoventilation: predictors and outcomes in childhood progressive neuromuscular disease. Arch Dis Child 2010:95:998-1003.

44. Phillips MF, Quinlivan RC, Edwards RH, et al. Changes in spirometry over time as a prognostic marker in patients with Duchenne muscular dystrophy. Am J Respir Crit Care Med 2001;164:2191-4.

45. Phillips MF, Smith PE, Carroll N, et al. Nocturnal oxygenation and prognosis in Duchenne muscular dystrophy. Am J Respir Crit Care Med 1999;160:198-202.

46. Fromageot C, Lofaso F, Annane D, et al. Supine fall in lung volumes in the assessment of diaphragmatic weakness in neuromuscular disorders. Arch Phys Med Rehabil 2001;82:123-8.

47. Hibbert ME, Lanigan A, Raven J, et al. Relation of armspan to height and the prediction of lung function. Thorax 1988;43:657-9.

48. Johnson BE, Westgate HD. Methods of predicting vital capacity in patients with thoracic scoliosis. J Bone Joint Surg Am 1970:52:1433-9.

49. Linderholm $\mathbf{H}$, Lindgren U. Prediction of spirometric values in patients with scoliosis. Acta Orthop Scand 1978; 49:469-74.

50. Tompsett J, Yousafzai AK, Filteau SM. The nutritional status of disabled children in Nigeria: a cross-sectional survey. Eur J Clin Nutr 1999;53:915-19.

51. Socrates C, Grantham-McGregor SM, Harknett SG, et al. Poor nutrition is a serious problem in children with cerebral palsy in Palawan, the Philippines. Int J Rehabil Res 2000;23:177-84. 
52. Yousafzai AK, Filteau SM, Wirz SL, et al. Comparison of armspan, arm length and tibia length as predictors of actual height of disabled and nondisabled children in Dharavi, Mumbai, India. Eur J Clin Nutr 2003;57:1230-4.

53. Gauld LM, Kappers J, Carlin JB, et al. Height prediction from ulna length. Dev Med Child Neurol 2004;46:475-80.

54. Gauld LM, Kappers J, Carlin JB, et al. Prediction of childhood pulmonary function using ulna length. Am J Respir Crit Care Med 2003;168:804-9.

55. Gauld LM, Boynton A, Betts GA, et al. Spirometry is affected by intelligence and behavior in Duchenne muscular dystrophy. Pediatr Pulmonol 2005:40:408-13.

56. Dohna-Schwake C, Ragette R, Teschler $\mathrm{H}$, et al. Predictors of severe chest infections in pediatric neuromuscular disorders. Neuromuscul Disord 2006;16:325-8.

57. Ragette R, Mellies U, Schwake C, et al. Patterns and predictors of sleep disordered breathing in primary myopathies. Thorax 2002:57:724-8.

58. Mellies U, Ragette R, Schwake C, et al. Daytime predictors of sleep disordered breathing in children and adolescents with neuromuscular disorders. Neuromuscul Disord 2003:13:123-8.

59. Toussaint $\mathbf{M}$, Steens $\mathbf{M}$, Soudon P. Lung function accurately predicts hypercapnia in patients with Duchenne muscular dystrophy. Chest 2007;131:368-75.

60. Hukins CA, Hillman DR. Daytime predictors of sleep hypoventilation in Duchenne muscular dystrophy. Am J Respir Crit Care Med 2000;161:166-70.

61. Hahn A, Bach JR, Delaubier A, et al. Clinical implications of maximal respiratory pressure determinations for individuals with Duchenne muscular dystrophy. Arch Phys Med Rehabil 1997;78:1-6.

62. Rafferty GF, Leech S, Knight L, et al. Sniff nasal inspiratory pressure in children. Pediatr Pulmonol 2000:29:468-75.

63. Stefanutti D, Benoist MR, Scheinmann P, et al. Usefulness of sniff nasal pressure in patients with neuromuscular or skeletal disorders. Am J Respir Crit Care Med 2000:162:1507-11

64. Nicot F, Hart N, Forin V, et al. Respiratory muscle testing: a valuable tool for children with neuromuscular disorders. Am J Respir Crit Care Med 2006:174:67-74

65. Lofaso F, Nicot F, Lejaille $M$, et al. Shiff nasal inspiratory pressure: what is the optimal number of sniffs? Eur Respir J 2006:27:980-2.

66. Bach JR, Saporito LR. Criteria for extubation and tracheostomy tube removal for patients with ventilatory failure. A different approach to weaning. Chest 1996:110:1566-71.

67. Bach JR, Goncalves MR, Hamdani I, et al. Extubation of patients with neuromuscular weakness: a new management paradigm. Chest 2010;137:1033-9.

68. Bach JR, Ishikawa Y, Kim H. Prevention of pulmonary morbidity for patients with Duchenne muscular dystrophy. Chest 1997:112:1024-8.

69. Sancho J, Servera E, Díaz J, et al. Predictors of ineffective cough during a chest infection in patients with stable amyotrophic lateral sclerosis. Am J Respir Crit Care Med 2007;175:1266-71.

70. Poponick JM, Jacobs I, Supinski G, et al. Effect of upper respiratory tract infection in patients with neuromuscular disease. Am J Respir Crit Care Med 1997; 156:659-64.

71. Gauld LM, Boynton A. Relationship between peak cough flow and spirometry in Duchenne muscular dystrophy. Pediatr Pulmonology 2005;39:457-60.

72. Bianchi C, Baiardi P. Cough peak flows: standard values for children and adolescents. Am J Phys Med Rehabil 2008;87:461-7.

73. Brouillette RT, Morielli A, Leimanis A, et al. Nocturnal pulse oximetry as an abbreviated testing modality for pediatric obstructive sleep apnea. Pediatrics 2000;105:405-12.

74. Paiva R, Krivec U, Aubertin G, et al. Carbon dioxide monitoring during longterm noninvasive respiratory support in children. Intensive Care Med 2009;35:1068-74.

75. Kirk VG, Flemons WW, Adams C, et al. Sleep-disordered breathing in Duchenne muscular dystrophy: a preliminary study of the role of portable monitoring. Pediatr Pulmonol 2000;29:135-40.

76. Kirk VG, Batuyong ED, Bohn SG. Transcutaneous carbon dioxide monitoring and capnography during pediatric polysomnography. Sleep 2006;29:1601-8.

77. Finder JD, Birnkrant D, Carl J, et al. Respiratory care of the patient with Duchenne muscular dystrophy: aTS consensus statement. Am J Respir Crit Care Med 2004; 170:456-65.

78. Ward S, Chatwin M, Heather S, et al. Randomised controlled trial of non-invasive ventilation (NIV) for nocturnal hypoventilation in neuromuscular and chest wall disease patients with daytime normocapnia. Thorax 2005;60:1019-24.

79. Kotterba S, Patzold T, Malin JP, et al. Respiratory monitoring in neuromuscular disease-capnography as an additional tool? Clin Neurol Neurosurg 2001; 103:87-91.

80. Wang CH, Finkel RS, Bertini ES, et al. Consensus statement for standard of care in spinal muscular atrophy. J Child Neurol 2007;22:1027-49.

81. Philpot J, Bagnall A, King C, et al. Feeding problems in merosin deficient congenital muscular dystrophy. Arch Dis Childhood 1999:80:542-7.

82. Aloysius A, Born P, Kinali M, et al. Swallowing difficulties in Duchenne muscular dystrophy: indications for feeding assessment and outcome of videofluroscopic swallow studies. Eur J Paediatr Neurol 2008;12:239-45.

83. Bagnall AK, Al-Muhaizea MA, Manzur AY. Feeding and speech difficulties in typical congenital nemaline myopathy. Adv Speech Lang Pathol 2006:8:7-16.

84. Shinonaga C, Fukuda M, Suzuki Y, et al. Evaluation of swallowing function in Duchenne muscular dystrophy. Dev Med Child Neurol 2008;50:478-80.
85. Armstrong A. Developing a breath-stacking system to achieve lung volume recruitment. Br J Nurs 2009;18:1166-9.

86. Bach JR, Mahajan K, Lipa B, et al. Lung insufflation capacity in neuromuscular disease. Am J Phys Med Rehabil 2008;87:720-5.

87. Dail CW. 'Glossopharyngeal breathing' by paralyzed patients; a preliminary report. Calif Med 1951:75:217-18

88. Dail CW, Affeldt JE, Collier CR. Clinical aspects of glossopharyngeal breathing; report of use by one hundred postpoliomyelitic patients. J Am Med Assoc 1955;158:445-9.

89. Bach JR. Mechanical insufflations-exsufflation. Comparison of peak expiratory flows with manually assisted and unassisted coughing techniques. Chest 1993;104:1553-62.

90. Massery M. Chest development as a component of normal motor development: implications for pediatric physical therapists. Pediatr Phys Ther 1991:3:3-8.

91. Bach JR, Baird JS, Plosky D, et al. Spinal muscular atrophy type 1: management and outcomes. Pediatr Pulmonol 2002;34:16-22.

92. Bach JR, Niranjan V, Weaver B. Spinal muscular atrophy type 1: a noninvasive respiratory management approach. Chest 2000;117:1100-5.

93. Gomez-Merino E, Bach JR. Duchenne muscular dystrophy: prolongation of life by noninvasive ventilation and mechanically assisted coughing. Am J Phys Med Rehabil 2002;81:411-15

94. Bach JR, Saltstein K, Sinquee D, et al. Long-term survival in Werdnig-Hoffmann disease. Am J Phys Med Rehabil 2007;86:339-45. quiz 46-8.

95. Oskoui M, Levy G, Garland CJ, et al. The changing natural history of spinal muscular atrophy type 1. Neurology 2007;69:1931-6.

96. Chatwin M, Bush A, Simonds AK. Outcome of goal-directed non-invasive ventilation and mechanical insufflation/exsufflation in spinal muscular atrophy type I. Arch Dis Childhood 2011:96:426-32.

97. Birnkrant DJ, Pope JF, Lewarski J, et al. Persistent pulmonary consolidation treated with intrapulmonary percussive ventilation: a preliminary report. Pediatr Pulmonol 1996:21:246-9.

98. Toussaint $\mathbf{M}$, De Win $H$, Steens $M$, et al. Effect of intrapulmonary percussive ventilation on mucus clearance in Duchenne muscular dystrophy patients: a preliminary report. Respir Care 2003:48:940-7.

99. Reardon CC, Christiansen D, Barnett ED, et al. Intrapulmonary percussive ventilation vs incentive spirometry for children with neuromuscular disease. Arch Pediatr Adolesc Med 2005;159:526-31.

100. Yuan N, Kane P, Shelton K, et al. Safety, tolerability, and efficacy of high-frequency chest wall oscillation in pediatric patients with cerebral palsy and neuromuscular diseases: an exploratory randomized controlled trial. J Child Neurol 2010;25:815-21.

101. Keating JM, Collins N, Bush A, et al. High frequency chest wall oscillation in a noninvasive ventilation dependent patient with spinal muscular atrophy type 1. Respir Care 2011:56:1840-3

102. Kang SW, Bach JR. Maximum insufflation capacity. Chest 2000:118:61-5

103. Kang SW, Kang YS, Moon JH, et al. Assisted cough and pulmonary compliance in patients with Duchenne muscular dystrophy. Yonsei Med J 2005;46:233-8.

104. Ishikawa Y, Bach JR, Komaroff $E$, et al. Cough augmentation in Duchenne muscular dystrophy. Am J Phys Med Rehabil 2008;87:726-30.

105. Brito MF, Moreira GA, Pradella-Hallinan M et al. Air stacking and ches compression increase peak cough flow in patients with Duchenne muscular dystrophy. (In Portuguese). J Bras Pneumol 2009;35:973-9.

106. Dohna-Schwake C, Ragette $\mathrm{R}$, Teschler $\mathrm{H}$, et al. IPPB-assisted coughing in neuromuscular disorders. Pediatr Pulmonol 2006;41:551-7

107. Nygren-Bonnier M, Markstrom A, Lindholm P, et al. Glossopharyngeal pistoning for lung insufflation in children with spinal muscular atrophy type II. Acta Paediatr 2009;98:1324-8.

108. Bach JR, Bianchi C, Vidigal-Lopes $\mathrm{M}$, et al. Lung inflation by glossopharyngeal breathing and 'air stacking' in Duchenne muscular dystrophy. Am J Phys Med Rehabil 2007;86:295-300.

109. Kang SW, Kang YS, Sohn HS, et al. Respiratory muscle strength and cough capacity in patients with Duchenne muscular dystrophy. Yonsei Med J 2006;47:184-90.

110. Toussaint M, Boitano LJ, Gathot V, et al. Limits of effective cough-augmentation techniques in patients with neuromuscular disease. Respir Care 2009;54:359-66.

111. Fauroux B, Guillemot N, Aubertin G, et al. Physiologic benefits of mechanical insufflation-exsufflation in children with neuromuscular diseases. Chest 2008:133:161-8.

112. Chatwin M, Ross $\mathrm{E}$, Hart $\mathrm{N}$, et al. Cough augmentation with mechanical insufflation/exsufflation in patients with neuromuscular weakness. Eur Respir J 2003;21:502-8.

113. Bach JR, Smith WH, Michaels J, et al. Airway secretion clearance by mechanica exsufflation for post-poliomyelitis ventilator-assisted individuals. Arch Phys Med Rehabil 1993; 74:170-7.

114. Chatwin M, Simonds AK. The addition of mechanical insufflation/exsufflation shortens airway-clearance sessions in neuromuscular patients with chest infection. Respir Care 2009;54:1473-9.

115. Miske LJ, Hickey EM, Kolb SM, et al. Use of the mechanical in-exsufflator in pediatric patients with neuromuscular disease and impaired cough. Chest 2004; 125:1406-12.

116. Vianello A, Corrado A, Arcaro G, et al. Mechanical insufflation-exsufflation improves outcomes for neuromuscular disease patients with respiratory tract infections. Am J Phys Med Rehabil 2005;84:83-8; discussion 89-91. 
117. Suri P, Burns SP, Bach JR. Pneumothorax associated with mechanical insufflations-exsufflation and related factors. Am J Phys Med Rehabil 2008:87:951-5.

118. Leith DE, Bradley M. Ventilatory muscle strength and endurance training. J App/ Physiol 1976;41:508-16.

119. Gozal D, Thiriet P. Respiratory muscle training in neuromuscular disease: long-term effects on strength and load perception. Med Sci Sports Exerc 1999;31:1522-7.

120. Martin AJ, Stern L, Yeates J, et al. Respiratory muscle training in Duchenne muscular dystrophy. Dev Med Child Neurol 1986:28:314-18.

121. Rodillo E, Noble-Jamieson CM, Aber V, et al. Respiratory muscle training in Duchenne muscular dystrophy. Arch Dis Childhood 1989;64:736-8.

122. Topin N, Matecki S, Le Bris S, et al. Dose-dependent effect of individualized respiratory muscle training in children with Duchenne muscular dystrophy. Neuromuscul Disord 2002:12:576-83.

123. Wanke T, Toifl $\mathrm{K}$, Merkle $\mathrm{M}$, et al. Inspiratory muscle training in patients with Duchenne muscular dystrophy. Chest 1994;105:475-82.

124. Koessler W, Wanke T, Winkler G, et al. 2 Years' experience with inspiratory muscle training in patients with neuromuscular disorders. Chest 2001;120:765-9.

125. Winkler G, Zifko U, Nader A, et al. Dose-dependent effects of inspiratory muscle training in neuromuscular disorders. Muscle Nerve 2000;23:1257-60.

126. Annane D, Quera-Salva MA, Lofaso F, et al. Mechanisms underlying effects of nocturnal ventilation on daytime blood gases in neuromuscular diseases. Eur Respir J 1999:13:157-62.

127. Nickol AH, Hart N, Hopkinson NS, et al. Mechanisms of improvement of respiratory failure in patients with restrictive thoracic disease treated with noninvasive ventilation. Thorax 2005;60:754-60.

128. Piper AJ, Sullivan CE. Effects of long-term nocturnal nasal ventilation on spontaneous breathing during sleep in neuromuscular and chest wall disorders. Eur Respir J 1996;9:1515-22

129. Hill NS, Eveloff SE, Carlisle CC, et al. Efficacy of nocturnal nasal ventilation in patients with restrictive thoracic disease. Am Rev Respir Dis 1992;145:365-71.

130. Mellies U, Ragette R, Dohna Schwake C, et al. Long-term noninvasive ventilation in children and adolescents with neuromuscular disorders. Eur Respir $\mathrm{J}$ 2003;22:631-6

131. Toussaint $\mathbf{M}$, Soudon $\mathbf{P}$, Kinnear $\mathbf{W}$. Effect of non-invasive ventilation on respiratory muscle loading and endurance in patients with Duchenne muscular dystrophy. Thorax 2008;63:430-4.

132. Mellies U, Dohna-Schwake C, Stehling F, et al. Sleep disordered breathing in spinal muscular atrophy. Neuromuscul Disord 2004;14:797-803.

133. Simonds AK, Ward $\mathrm{S}$, Heather $\mathrm{S}$, et al. Outcome of paediatric domiciliary mask ventilation in neuromuscular and skeletal disease. Eur Respir J 2000;16:476-81.

134. Young HK, Lowe A, Fitzgerald DA, et al. Outcome of noninvasive ventilation in children with neuromuscular disease. Neurology 2007;68:198-201.

135. Raphael JC, Chevret S, Chastang C, et al. Randomised trial of preventive nasal ventilation in Duchenne muscular dystrophy. French Multicentre Cooperative Group on Home Mechanical Ventilation Assistance in Duchenne de Boulogne Muscular Dystrophy. Lancet 1994;343:1600-4.

136. Dohna-Schwake C, Podlewski P, Voit T, et al. Non-invasive ventilation reduces respiratory tract infections in children with neuromuscular disorders. Pediatr Pulmonol 2008:43:67-71.

137. Katz S, Selvadurai H, Keilty K, et al. Outcome of non-invasive positive pressure ventilation in paediatric neuromuscular disease. Arch Dis Child 2004:89:121-4.

138. Bach JR, Bianchi C. Prevention of pectus excavatum for children with spinal muscular atrophy type 1. Am J Phys Med Rehabil 2003;82:815-19.

139. Petrone A, Pavone M, Testa MBC, et al. Noninvasive ventilation in children with spinal muscular atrophy types 1 and 2. Am J Phys Med Rehabil 2007:86:216-21.

140. Simonds AK, Muntoni F, Heather $S$, et al. Impact of nasal ventilation on survival in hypercapnic Duchenne muscular dystrophy. Thorax 1998:53:949-52.

141. Eagle M, Baudouin SV, Chandler C, et al. Survival in Duchenne muscular dystrophy: improvements in life expectancy since 1967 and the impact of home nocturnal ventilation. Neuromuscul Disord 2002;12:926-9.

142. Jeppesen J, Green A, Steffensen BF, et al. The Duchenne muscular dystrophy population in Denmark, 1977-2001: prevalence, incidence and survival in relation to the introduction of ventilator use. Neuromuscul Disord 2003;13:804-12.

143. Toussaint M, Steens M, Wasteels G, et al. Diurnal ventilation via mouthpiece: survival in end-stage Duchenne patients. Eur Respir J 2006;28:549-55.

144. Soudon P, Steens M, Toussaint M. A comparison of invasive versus noninvasive full-time mechanical ventilation in Duchenne muscular dystrophy. Chronic Respir Dis 2008;5:87-93

145. Lofaso F, Brochard L, Touchard D, et al. Evaluation of carbon dioxide rebreathing during pressure support ventilation with airway management system (BiPAP) devices. Chest 1995:108:772-8.

146. Fauroux B, Leroux K, Desmarais G, et al. Performance of ventilators for noninvasive positive-pressure ventilation in children. Eur Respir J 2008;31:1300-7.

147. Chatwin M, Nickol AH, Morrell MJ, et al. Randomised trial of inpatient versus outpatient initiation of home mechanical ventilation in patients with nocturnal hypoventilation. Respir Med 2008;102:1528-35.

148. Gonzalez J, Sharshar T, Hart N, et al. Air leaks during mechanical ventilation as a cause of persistent hypercapnia in neuromuscular disorders. Intensive Care Med 2003:29:596-602.

149. Meyer TJ, Pressman MR, Benditt J, et al. Air leaking through the mouth during nocturnal nasal ventilation: effect on sleep quality. Sleep 1997;20:561-9.
150. Orlikowski D, Mroue G, Prigent $\mathrm{H}$, et al. Automatic air-leak compensation in neuromuscular patients: a feasibility study. Respir Med 2009;103:173-9.

151. Fauroux B, Lavis JF, Nicot $F$, et al. Facial side effects during noninvasive positive pressure ventilation in children. Intensive Care Med 2005;31:965-9.

152. Villa MP, Pagani J, Ambrosio R, et al. Mid-face hypoplasia after long-term nasa ventilation. Am J Respir Crit Care Med 2002:166:1142-3.

153. Baydur A, Kanel G. Tracheobronchomalacia and tracheal hemorrhage in patients with Duchenne muscular dystrophy receiving long-term ventilation with uncuffed tracheostomies. Chest 2003:123:1307-11.

154. Iodice FG, Salzano M, Marri M, et al. Tracheobronchial haemorrhage in patients with neuromuscular disorders. Respir Med 2005;99:1613-15.

155. Bach JR, Rajaraman R, Ballanger $F$, et al. Neuromuscular ventilatory insufficiency: effect of home mechanical ventilator use $\mathrm{v}$ oxygen therapy on pneumonia and hospitalization rates. Am J Phys Med Rehabil 1998:77:8-19.

156. Bach JR. A comparison of long-term ventilatory support alternatives from the perspective of the patient and care giver. Chest 1993;104:1702-6.

157. Markstrom A, Sundell K, Lysdahl M, et al. Quality-of-life evaluation of patients with neuromuscular and skeletal diseases treated with noninvasive and invasive home mechanical ventilation. Chest 2002;122:1695-700.

158. Hill NS, Redline S, Carskadon MA et al. Sleep-disordered breathing in patients with Duchenne muscular dystrophy using negative pressure ventilators. Chest 1992;102:1656-62.

159. Smith PE, Edwards RH, Calverley PM. Oxygen treatment of sleep hypoxaemia in Duchenne muscular dystrophy. Thorax 1989;44:997-1001.

160. Padman R, Lawless S, Von Nessen S. Use of BiPAP by nasal mask in the treatment of respiratory insufficiency in pediatric patients: preliminary investigation. Pediatr Pulmonol 1994;17:119-23.

161. Niranjan V, Bach JR. Noninvasive management of pediatric neuromuscular ventilatory failure. Crit Care Med 1998;26:2061-5.

162. Vianello A, Bevilacqua M, Arcaro G, et al. Non-invasive ventilatory approach to treatment of acute respiratory failure in neuromuscular disorders. A comparison with endotracheal intubation. Intensive Care Med 2000;26:384-90.

163. Piastra M, Antonelli M, Caresta E, et al. Noninvasive ventilation in childhood acute neuromuscular respiratory failure: a pilot study. Respiration 2006:73:791-8.

164. Servera E, Sancho J, Zafra MJ, et al. Alternatives to endotracheal intubation for patients with neuromuscular diseases. Am J Phys Med Rehabil 2005;84:851-7.

165. Pope JF, Birnkrant DJ. Noninvasive ventilation to facilitate extubation in a pediatric intensive care unit. J Intensive Care Med 2000;15:99-103.

166. Bertorini TE. Myoglobinuria, malignant hyperthermia, neuroleptic malignant syndrome and serotonin syndrome. Neurol Clin 1997;15:649-71.

167. Larsen UT, Juhl B, Hein-Sorensen 0, et al. Complications during anaesthesia in patients with Duchenne's muscular dystrophy (a retrospective study). Can J Anaesth 1989;36:418-22.

168. Yemen TA, McClain C. Muscular dystrophy, anesthesia and the safety of inhalational agents revisited; again. Paediatr Anaesth 2006;16:105-8.

169. Seay AR, Ziter FA, Thompson JA. Cardiac arrest during induction of anesthesia in Duchenne muscular dystrophy. J Pediatr 1978:93:88-90.

170. Tobias JD. A review of intrathecal and epidural analgesia after spinal surgery in children. Anesth Analg 2004;98:956-65, table of contents.

171. lannaccone ST, Owens $\mathrm{H}$, Scott J, et al. Postoperative malnutrition in Duchenne muscular dystrophy. J Child Neurol 2003;18:17-20.

172. Jenkins JG, Bohn D, Edmonds JF, et al. Evaluation of pulmonary function in muscular dystrophy patients requiring spinal surgery. Crit Care Med 1982;10:645-9

173. Silva WJ, Rucker RW, Ewald BA. Preoperative pulmonary assessment in pediatric neuromuscular disease. Anesthesiol Rev 1982:9:35-42.

174. Yuan N, Skaggs DL, Dorey F, et al. Preoperative predictors of prolonged postoperative mechanical ventilation in children following scoliosis repair. Pediatr Pulmonol 2005; 40:414-19.

175. Udink ten Cate FE, van Royen BJ, van Heerde M, et al. Incidence and risk factors of prolonged mechanical ventilation in neuromuscular scoliosis surgery. $J$ Pediatr Orthop B 2008;17:203-6.

176. Takaso M, Nakazawa T, Imura T, et al. Preoperative inspiratory muscle training for patients with severe scoliosis and high-risk pulmonary dysfunction in duchenne muscular dystrophy. Eur J Orthop Surg Traumatol 2010;20:113-21.

177. Marsh A, Edge G, Lehovsky J. Spinal fusion in patients with Duchenne's muscula dystrophy and a low forced vital capacity. Eur Spine J 2003:12:507-12.

178. Harper CM, Ambler G, Edge G. The prognostic value of pre-operative predicted forced vital capacity in corrective spinal surgery for Duchenne's muscular dystrophy. Anaesthesia 2004;59:1160-2

179. Gill I, Eagle M, Mehta JS, et al. Correction of neuromuscular scoliosis in patients with preexisting respiratory failure. Spine 2006;31:2478-83.

180. Yuan N, Skaggs DL, Davidson Ward SL, et al. Preoperative polysomnograms and infant pulmonary function tests do not predict prolonged postoperative mechanica ventilation in children following scoliosis repair. Pediatr Pulmonol 2004:38:256-60.

181. DiMeglio A, Canavese F, Charles YP. Growth and adolescent idiopathic scoliosis: when and how much? J Pediatr Orthop 2011;31(1 Suppl):S28-36.

182. Bayar B, Uygur F, Bayar K, et al. The short-term effects of an exercise programme as an adjunct to an orthosis in neuromuscular scoliosis. Prosthet Orthot Int 2004:28:273-7.

183. Miller G, O'Connor J. Spinal bracing and respiratory function in Duchenne muscular dystrophy. Clin Pediatr (Phila) 1985;24:94. 
184. Noble-Jamieson CM, Heckmatt JZ, Dubowitz V, et al. Effects of posture and spinal bracing on respiratory function in neuromuscular disease. Arch Dis Child 1986;61:178-81.

185. Tangsrud SE, Carlsen KC, Lund-Petersen I, et al. Lung function measurements in young children with spinal muscle atrophy; a cross sectional survey on the effect of position and bracing. Arch Dis Child 2001:84:521-4.

186. Seeger BR, Sutherland AD, Clark MS. Orthotic management of scoliosis in Duchenne muscular dystrophy. Arch Phys Med Rehabil 1984;65:83-6.

187. Evans GA, Drennan JC, Russman BS. Functional classification and orthopaedic management of spinal muscular atrophy. J Bone Joint Surg Br 1981;63B:516-22.

188. Cambridge W, Drennan JC. Scoliosis associated with Duchenne muscular dystrophy. J Pediatr Orthop 1987:7:436-40.

189. Colbert AP, Craig C. Scoliosis management in Duchenne muscular dystrophy: prospective study of modified Jewett hyperextension brace. Arch Phys Med Rehab 1987:68:302-4.

190. Chng SY, Wong YQ, Hui JH, et al. Pulmonary function and scoliosis in children with spinal muscular atrophy types II and III. J Paediatrics Child Health 2003;39:673-6.

191. Shapiro F, Sethna N, Colan S, et al. Spinal fusion in Duchenne muscular dystrophy: a multidisciplinary approach. Muscle Nerve 1992;15:604-14.

192. Velasco MV, Colin AA, Zurakowski D, et al. Posterior spinal fusion for scoliosis in duchenne muscular dystrophy diminishes the rate of respiratory decline. Spine 2007;32:459-65.

193. Miller RG, Chalmers AC, Dao $\mathrm{H}$, et al. The effect of spine fusion on respiratory function in Duchenne muscular dystrophy. Neurology 1991;41:38-40.

194. Galasko CS, Delaney C, Morris P. Spinal stabilisation in Duchenne muscular dystrophy. J Bone Joint Surg Br 1992;74(2):210-14.

195. Kennedy JD, Staples AJ, Brook PD, et al. Effect of spinal surgery on lung function in Duchenne muscular dystrophy. Thorax 1995;50:1173-8.

196. Kinali M, Messina S, Mercuri E, et al. Management of scoliosis in Duchenne muscular dystrophy: a large 10-year retrospective study. Dev Med Child Neurol 2006:48:513-18

197. Piasecki J0, Mahinpour S, Levine DB. Long-term follow-up of spinal fusion in spinal muscular atrophy. Clin Orthop Relat Res 1986:44-54.

198. Brown JC, Zeller JL, Swank SM, et al. Surgical and functional results of spine fusion in spinal muscular atrophy. Spine 1989;14:763-70.

199. Takaso M, Nakazawa T, Imura T, et al. Surgical management of severe scoliosis with high risk pulmonary dysfunction in Duchenne muscular dystrophy: patient function, quality of life and satisfaction. Int Orthop 2010;34:695-702

200. Durkin ET, Schroth MK, Helin M, et al. Early laparoscopic fundoplication and gastrostomy in infants with spinal muscular atrophy type I. J Pediatr Surg 2008;43:2031-7.

201. Kawahara H, Mitani Y, Nose K, et al. Should fundoplication be added at the time of gastrostomy placement in patients who are neurologically impaired? J Pediatr Surg 2010;45:2373-6.

202. Viswanath $\mathbf{N}$, Wong D, Channappa D, et al. Is prophylactic fundoplication necessary in neurologically impaired children? Eur J Pediatr Surg 2010;20:226-9.

203. Birnkrant DJ, Pope JF, Martin JE, et al. Treatment of type I spinal muscular atrophy with noninvasive ventilation and gastrostomy feeding. Pediatr Neurol 1998;18:407-10.

204. Bach JR, Gonzalez M, Sharma A, et al. Open gastrostomy for noninvasive ventilation users with neuromuscular disease. Am J Phys Med Rehabil 2010;89:1-6

205. Sy K, Mahant S, Taback N, et al. Enterostomy tube placement in children with spinal muscular atrophy type 1. J Pediatr 2006;149:837-9.

206. Panitch HB. Viral respiratory infections in children with technology dependence and neuromuscular disorders. Pediatr Infect Dis J 2004:23(11 Suppl):S222-7.

207. Jaffe KM, McDonald CM, Ingman E, et al. Symptoms of upper gastrointestinal dysfunction in Duchenne muscular dystrophy: case-control study. Arch Phys Med Rehabil 1990;71:742-4.

208. Pane M, Vasta I, Messina S, et al. Feeding problems and weight gain in Duchenne muscular dystrophy. Eur J Paediatr Neurol 2006:10:231-6.

209. Willig TN, Paulus J, Lacau Saint Guily J, et al. Swallowing problems in neuromuscular disorders. Arch Phys Med Rehabil 1994;75:1175-81.

210. Hill M, Hughes T, Milford C. Treatment for swallowing difficulties (dysphagia) in chronic muscle disease. Cochrane Database Syst Rev 2004;(2):CD004303.

211. Yuan N, Wang CH, Trela A, et al. Laparoscopic Nissen fundoplication during gastrostomy tube placement and noninvasive ventilation may improve survival in type I and severe type II spinal muscular atrophy. J Child Neurol 2007;22:727-31.

212. Ramelli GP, Aloysius A, King C, et al. Gastrostomy placement in paediatric patients with neuromuscular disorders: indications and outcome. Dev Med Child Neurol 2007:49:367-71

213. Seguy D, Michaud L, Guimber D, et al. Efficacy and tolerance of gastrostomy feeding in pediatric forms of neuromuscular diseases. JPEN J Parenteral Enteral Nutr 2002;26:298-304.

214. Martigne L, Seguy D, Pellegrini N, et al. Efficacy and tolerance of gastrostomy feeding in Duchenne muscular dystrophy. Clin Nutr 2010;29:60-4.

215. Wilkesmann A, Ammann RA, Schildgen 0, et al. Hospitalized children with respiratory syncytial virus infection and neuromuscular impairment face an increased risk of a complicated course. Pediatr Infect Dis J 2007;26:485-91.

216. Thorburn K. Pre-existing disease is associated with a significantly higher risk of death in severe respiratory syncytial virus infection. Arch Dis Child 2009;94:99-103
217. Keren R, Zaoutis TE, Bridges CB, et al. Neurological and neuromuscular disease as a risk factor for respiratory failure in children hospitalized with influenza infection. JAMA 2005:294:2188-94

218. Doug M, Adi Y, Williams J, et al. Transition to adult services for children and young people with palliative care needs: a systematic review. Arch Dis Child 2011;96:78-84

219. Hill ME, Phillips MF. Service provision for adults with long-term disability: a review of services for adults with chronic neuromuscular conditions in the United Kingdom. Neuromuscul Disord 2006:16:107-12.

220. Craig F, Rajapakse CD, McNamara K, et al. Investing in primary care as the bridge for transitional care. Arch Dis Child 2011;96:1-2

221. Birnkrant DJ. New challenges in the management of prolonged survivors of pediatric neuromuscular diseases: a pulmonologist's perspective. Pediatr Pulmonol 2006;41:1113-17.

222. Rahbek J, Werge B, Madsen A, et al. Adult life with Duchenne muscular dystrophy: observations among an emerging and unforeseen patient population. Pediatr Rehabil 2005:8:17-28.

223. Stewart D. Transition to adult services for young people with disabilities: current evidence to guide future research. Dev Med Child Neurol 2009;51(Suppl 4):169-73.

224. Engel JM, Kartin D, Carter GT, et al. Pain in youths with neuromuscular disease. Am J Hosp Palliat Med 2009;26:405-12.

225. Rotimi F, Childs AM, Potrata B. A pilot study to evaluate current practices of transition of young adults from paediatric to adult neuromuscular (NM) clinics. Eur $J$ Neurol 2010;17:532

226. Iannaccone ST, Hynan LS. Reliability of 4 outcome measures in pediatric spinal muscular atrophy. Arch Neurol 2003:60:1130-6.

227. Bray P, Bundy AC, Ryan MM, et al. Health-related quality of life in boys with duchenne muscular dystrophy: agreement between parents and their sons. J Child Neurol 2010;25:1188-94.

228. lannaccone ST, Hynan LS, Morton A, et al. The PedsOL in pediatric patients with spinal muscular atrophy: feasibility, reliability, and validity of the pediatric quality of life Inventory generic core scales and neuromuscular Module. Neuromuscul Disord 2009;19:805-12.

229. Bach JR, Campagnolo DI, Hoeman S. Life satisfaction of individuals with Duchenne muscular dystrophy using long-term mechanical ventilatory support. Am J Phys Med Rehabil 1991;70:129-35.

230. Bach JR. Ventilator use by muscular dystrophy association patients. Arch Phys Med Rehabil 1992;73:179-83.

231. Bach JR, Vega J, Majors J, et al. Spinal muscular atrophy type 1 quality of life. Am J Phys Med Rehabil 2003;82:137-42.

232. Hardart MK, Truog D. Spinal muscular atrophy-type 1. Arch Dis Child 2003:88:848-50.

233. Sakakihara Y, Kubota M, Kim S, et al. Long-term ventilator support in patients with Werdnig-Hoffmann disease. Pediatr Int 2000;42:359-63.

234. Bothwell J, Dooley J, Gordon K, et al. Duchenne muscular dystrophy: parenta perceptions. Clin Pediatr 2002;41:105-9.

235. Mah JK, Thannhauser JE, Kolski H, et al. Parental stress and quality of life in children with neuromuscular disease. Pediatr Neurol 2008:39:102-7.

236. Brooks D, King A, Tonack $M$, et al. User perspectives on issues that influence the quality of daily life of ventilator-assisted individuals with neuromuscular disorders. Can Respir J 2004;11:547-54.

237. Kohler M, Clarenbach CF, Boni L, et al. Quality of life, physical disability, and respiratory impairment in Duchenne muscular dystrophy. Am J Respir Crit Care Med 2005; 172:1032-6.

238. Vuillerot C, Hodgkinson I, Bissery A, et al. Self-perception of quality of life by adolescents with neuromuscular diseases. J Adolesc Health 2010:46:70-6.

239. Jeppesen J, Madsen A, Marquardt J, et al. Living and ageing with spinal muscula atrophy type 2: observations among an unexplored patient population. Dev Neurorehabil 2010;13:10-18.

240. Grootenhuis MA, de Boone J, van der Kooi AJ. Living with muscular dystrophy: health related quality of life consequences for children and adults. Health Qual Life Outcomes 2007;5:31.

241. Ahlstrom G, Gunnarsson LG. Disability and quality of life in individuals with muscular dystrophy. Scand J Rehabil Med 1996:28:147-57.

242. Bach JR. Medical considerations of long-term survival of Werdnig-Hoffmann disease. Am J Phys Med Rehabil 2007;86:349-55.

243. Bostrom K, Ahlstrom G. Quality of life in patients with muscular dystrophy and their next of kin. Int J Rehabil Res 2005;28:103-9.

244. Narayanaswami P, Bertorini TE, Pourmand R, et al. Long-term tracheostomy ventilation in neuromuscular diseases: patient acceptance and quality of life. Neurorehabil Neural Repair 2000;14:135-9.

245. Read J, Simonds A, Kinali $M$, et al. Sleep and well-being in young men with neuromuscular disorders receiving non-invasive ventilation and their carers. Neuromuscul Disord 2010:20:458-63.

246. Geevasinga N, Ryan MM. Physician attitudes towards ventilatory support for spina muscular atrophy type 1 in Australasia. J Paediatrics Child Health 2007;43:790-4.

247. Hardart MK, Burns JP, Truog RD. Respiratory support in spinal muscular atrophy type I: a survey of physician practices and attitudes. Pediatrics 2002:110:e24.

248. Dybwik K, Tollali T, Nielsen EW, et al. Why does the provision of home mechanica ventilation vary so widely? Chronic Respir Dis 2010;7:67-73.

249. Gibson B. Long-term ventilation for patients with Duchenne muscular dystrophy: physicians' beliefs and practices. Chest 2001;119:940-6. 
250. Gilgoff I, Prentice W, Baydur A. Patient and family participation in the management of respiratory failure in Duchenne's muscular dystrophy. Chest 1989;95:519-24.

251. Kinali M, Manzur AY, Mercuri E, et al. UK physicians' attitudes and practices in long-term non-invasive ventilation of Duchenne muscular dystrophy. Pediatr Rehabil 2006:9:351-64

252. Miller JR, Colbert AP, Osberg JS. Ventilator dependency: decision-making, daily functioning and quality of life for patients with Duchenne muscular dystrophy. Dev Med Child Neurol 1990:32:1078-86.

253. Yates K, Festa M, Gillis J, et al. Outcome of children with neuromuscular disease admitted to paediatric intensive care. Arch Dis Childhood 2004;89:170-5.

254. Kenneson A, Bobo JK. The effect of caregiving on women in families with Duchenne/Becker muscular dystrophy. Health Soc Care Community 2010;18:520-8.

255. Thompson RJ Jr, Zeman JL, Fanurik D, et al. The role of parent stress and coping and family functioning in parent and child adjustment to Duchenne muscular dystrophy. J Clin Psychol 1992:48:11-19.

256. Abi Daoud MS, Dooley JM, Gordon KE. Depression in parents of children with Duchenne muscular dystrophy. Pediatr Neurol 2004;31:16-19.

257. Erby LH, Rushton C, Geller G. 'My son is still walking': stages of receptivity to discussions of advance care planning among parents of sons with Duchenne muscular dystrophy. Semin Pediatr Neurol 2006;13:132-40.

258. Yilmaz 0, Yildirim SA, Oksuz C, et al. Mothers' depression and health-related quality of life in neuromuscular diseases: role of functional independence level of the children. Pediatrics Int 2010;52:648-52.

259. Hodges L, Dibb B. Social comparison within self-help groups: views of parents of children with Duchenne muscular dystrophy. J Health Psychol 2010;15:483-92.

260. Drummond GB, Duncan MK. Abdominal pressure during laparoscopy: effects of fentanyl. Br J Anaesth 2002;88:384-8.

261. Fraser LK, Aldridge J, Manning S, et al. Hospice provision and usage amongst young people with neuromuscular disease in the United Kingdom. Eur J Paediatr Neurol 2011;15:326-30

262. Abresch RT, Carter GT, Jensen MP, et al. Assessment of pain and health-related quality of life in slowly progressive neuromuscular disease. Am J Hosp Palliat Med 2002;19:39-48.

263. Zebracki K, Drotar D. Pain and activity limitations in children with Duchenne or Becker muscular dystrophy. Dev Med Child Neurol 2008;50:546-52.

264. UKHoL Decision. Gillick versus West Norfolk and Wisbech Area Health Authority. 1985. http://www.bailii.org/uk/cases/UKHL/1985/7.html

265. Mental Capacity Act. Code of practice, 2007. England, 2005. http://www.legislation. gov.uk/ukpga/2005/9/contents

266. Wiener L, Ballard E, Brennan T, et al. How I wish to be remembered: the use of an advance care planning document in adolescent and young adult populations. $J$ Palliat Med 2008;11:1309-13.

267. Gilgoff IS, Kahlstrom E, MacLaughlin $E$, et al. Long-term ventilatory support in spinal muscular atrophy. J Pediatr 1989;115:904-9.

268. Penner L, Cantor RM, Siegel L. Joseph's wishes: ethical decision-making in Duchenne muscular dystrophy. Mt Sinai J Med 2010;77:394-7.

269. Dawson S, Kristjanson LJ. Mapping the journey: family carers' perceptions of issues related to end-stage care of individuals with muscular dystrophy or motor neurone disease. J Palliat Care 2003;19:36-42.

270. Parker D, Maddocks I, Stern LM. The role of palliative care in advanced muscular dystrophy and spinal muscular atrophy. J Paediatr Child Health 1999;35:245-50.

\section{APPENDIX 1 \\ GUIDELINE COMMITTEE MEMBERSHIP AND DECLARED CONFLICT OF INTEREST}

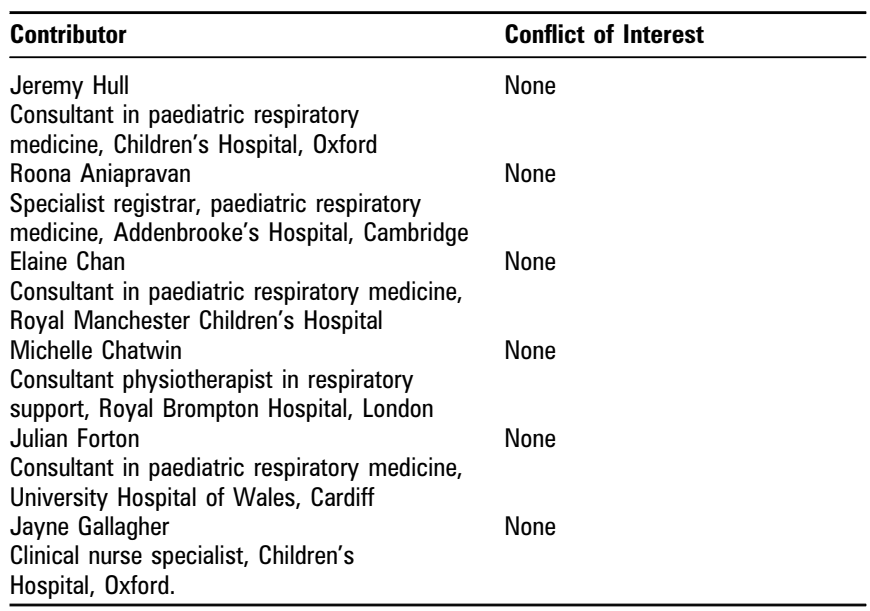

Continued
Continued

Contributor

Conflict of Interest

Neil Gibson (representing the Royal

None

College of Paediatric and Child Health

Consultant in paediatric respiratory

medicine, Royal Hospital for Sick

Children, Glasgow

Jill Gordon

Consultant community paediatrician,

Suffolk Primary Health Care Trust

Imelda Hughes

Consultant paediatric neurologist,

Royal Manchester Children's Hospital

Renee McCulloch

Consultant in paediatric palliative

medicine, NBK Hospital, Ministry of

Health, State of Kuwait

Senior clinical lecturer, Great Ormond

Street Hospital and Institute of Child

Health, London

Rob Ross Russell

None

Consultant in paediatric respiratory

medicine and paediatric intensive care,

Cambridge University Hospitals

Anita Simonds

Consultant in respiratory medicine,

Royal Brompton and Harefield

Hospital, London

Sian Haigh

Lay member and parent

None

None

None

The Guideline Committee would like to thank Dr Ingrid Du Rand Darwood for her advice on guideline methodology.

\section{APPENDIX 2 NEUROMUSCULAR CONDITIONS COVERED BY THE GUIDELINE}

Neuromuscular conditions are disorders of the 'motor unit' which comprises the anterior horn cell, peripheral nerve, neuromuscular junction and muscle. The cardinal feature of these conditions is muscle weakness. With severe muscle weakness there is usually secondary skeletal deformity.

The majority of children with neuromuscular disease have genetic conditions. In the past two decades there has been vast progress in the elucidation of the genetic and molecular pathology of neuromuscular conditions. Although there are currently trials of genetic disease-modifying treatment in DMD the only disease-modifying treatments currently in use in genetic neuromuscular disorders are steroids in DMD and enzyme replacement therapy in Pompe disease. Some of the congenital myasthenic syndromes will respond to symptomatic treatment with drugs which have an effect on neuromuscular transmission. Some children have acquired neuromuscular disease, such as inflammatory neuropathy, inflammatory myopathy or myasthenia gravis, all of which are potentially treatable but respiratory complications may occur at the time of weakness.

Involvement of respiratory, bulbar and abdominal wall muscles in these conditions causes a number of respiratory complications. Secondary skeletal abnormalities such as scoliosis or spinal rigidity also have an impact on the respiratory system. The differential involvement of these muscles in different neuromuscular disorders produces different patterns of respiratory complications in different conditions Respiratory complications are inevitable in severe progressive neuromuscular disorders and tend to occur with advanced weakness. In some conditions respiratory complications are less predictable and may, for example, be intermittent in congenita myasthenic syndromes or occur in patients who are still ambulant in Pompe's disease or some congenital myopathies. Respiratory complications result in a significant burden of morbidity in children with neuromuscular disorders and are often the lifelimiting aspect of these conditions. These complications are largely predictable and knowledge of individual conditions and their effect on the respiratory system can facilitate management.

Presentation with severe respiratory compromise may be as early as the neonatal period. Conditions which are particularly likely to present with respiratory failure in the neonatal period are congenital myopathies, especially myotubular myopathy and nemaline myopathy, congenital muscular dystrophy and congenital myasthenic syndromes.

\section{Spinal muscular atrophy}

The most common type of spinal muscular atrophy (SMA) is associated with homozygous deletions of exons 7 and 8 of the SMN1 gene or other rearrangements of this gene. Children with this condition have normal cognition and no facial weakness (at least in the period before there is respiratory failure). There is limb weakness which affects arms $>$ legs and proximal $>$ distal muscles. Intercostal muscles are affected 
with relative sparing of the diaphragm. Bulbar muscles are affected. Classification into types 1, 2 and 3 depends on best motor achievement; type 1 never sit independently, type 2 sit independently but do not stand independently, type 3 achieve independent walking. The spectrum of severity is however a continuum leading Victor Dubowitz to propose a decimal scale within each type (eg, type 1.0-1.9). ${ }^{1}$ The degree of respiratory involvement largely parallels motor involvement. Because of the prominent bulbar and intercostal muscle weakness, pneumonia is common, either aspiration or infection due to reduced secretion clearance.

Type I: in the past the vast majority died before the age of 2 years, mainly due to respiratory complications. loos and colleagues distinguish between those with onset in the first 3 months of life whose prognosis is worse than those with an onset between 3 and 6 months. ${ }^{2}$

Type II: a substantial number will develop respiratory complications during childhood; again these may be influenced by severity. Zerres and colleagues in a cohort of 168 patients reported pneumonia in the first 5 years in $28 \%$ of patients who achieved sitting only and $24 \%$ who achieved standing and breathing difficulties in the first 5 years in $12 \%$ who had sitting only and none in those who had achieved standing. ${ }^{3}$ loos and colleagues report a requirement for nocturnal non-invasive ventilation in $38 \%$, and tracheostomy in $15 \%$ of their cohort of 100 patients with type II SMA. ${ }^{2}$

Type III: patients have preservation of respiratory function until the teens followed by a decline in vital capacity but do not appear to have significant respiratory complications in childhood. ${ }^{2}$

\section{SMA with respiratory distress}

SMA with respiratory distress (SMARD) is a genetically heterogeneous condition with respiratory failure due to diaphragmatic weakness. SMARD1 is autosomal recessive and due to mutations in the IGHMBP2 gene. This condition usually presents with respiratory failure between the ages of 6 weeks and 6 months, often with eventration of the diaphragm. ${ }^{4}$ By contrast with SMA, there is prominent diaphragmatic weakness. Subsequently limb weakness, affecting distal more than proximal muscles and arms more than legs, develops.

\section{Duchenne/Becker muscular dystrophy}

Duchenne muscular dystrophy (DMD) and Becker muscular dystrophy (BMD) are X-linked conditions caused by mutations in the dystrophin gene. Dystrophin is a component of the sarcolemma of muscle whose function appears to be limitation of contraction-induced damage of the muscle cells. DMD and BMD represent different degrees of severity, although within each of these there is a range of severity. In DMD there is complete loss of dystrophin function with an earlier age of onset, more rapid progression and loss of independent ambulation by 13 years of age. In BMD there is some preservation of dystrophin function, age of onset is later, progression slower and independent ambulation is possible beyond 16 years of age.

$\mathrm{DMD}$ is the single most common neuromuscular condition in paediatric practice. Onset is by 5 years, motor development is usually but not always delayed, global developmental delay is common. There is progressive symmetrical proximal weakness affecting legs >arms. There is pseudo-hypertrophy, especially of calf muscles but temporalis, masseter and forearm muscles may be involved. Intellectual impairment is common but only occasionally is it severe and it is not progressive. Cardiomyopathy in DMD usually occurs when there is advanced skeletal muscle weakness but may occur earlier, and in BMD may occur with mild weakness; with some dystrophin mutations cardiomyopathy may occur without skeletal muscle weakness. Lifespan has increased decade on decade from the 1960s, and median life expectancy now exceeds 25 years. ${ }^{5}$ In DMD vital capacity declines rapidly between ages 10 and 20 years with the rate of decline slowing down after age 20. In one cohort study, the mean age at which mechanical ventilation was started was 20 years (range 14-31 years). ${ }^{6}$ Respiratory problems are not anticipated in childhood in BMD. Female carriers of dystrophin mutations may manifest muscle weakness which is usually not severe in childhood. A severe DMD-like phenotype may occur rarely and is usually associated with chromosomal translocation.

\section{Effect of steroids in DMD}

A Cochrane review of trials of the use of corticosteroids concluded that steroids improve muscle strength and function over 6 months and stabilise muscle strength and function for up to 2 years. ${ }^{7}$ Vital capacity was measured in four studies and showed a mean improvement at 6 months of $0.14-0.17$ litres. $^{7}$ Non-randomised studies suggest a long-term benefit on respiratory function. Biggar and colleagues report a significant difference in vital capacity between boys treated with deflazacort and untreated boys at 10, 15 and 18 years of age, which increased with time. At 18 years of age, $46 \%$ of the untreated group and none of the treated group required nocturnal non-invasive ventilation. ${ }^{8}$

\section{Limb girdle muscular dystrophy}

This is a group of genetically heterogeneous conditions. Classification is genetic. Ten per cent are autosomal dominant, for which there are currently three known genes (LGMD 1A-C); $90 \%$ are autosomal recessive, for which there are currently 15 known genes (LGMD2A-0). All have spectrum of severity with onset in childhood or adult life. Childhood-onset limb girdle muscular dystrophy is uncommon and when it occurs, has a similar phenotype to DMD/BMD. Respiratory problems occur after loss of ambulation when there is advanced limb weakness.

\section{Facioscapulohumeral muscular dystrophy}

Facioscapulohumeral (FSH) muscular dystrophy is an autosomal dominant disorder associated with a deletion within the D4Z4 repeat array on chromosome 4q. Age of onset varies and there may be intrafamilial variation. Initially there is facial weakness and weakness of the periscapular muscles causing poor scapular fixation and scapular winging. Subsequently there is proximal arm and ankle dorsiflexion weakness and later pelvic girdle weakness.

In 2-5\% of all FSH muscular dystrophy there is a severe form with infantile onset, facial weakness and marked lumbar lordosis, associated with sensorineural deafness and Coat's disease (retinal vasculopathy). Respiratory insufficiency requiring ventilatory support is very rare (approximately $1 \%$ of patients) in FSH muscular dystrophy; the majority of subjects requiring support have a disease onset before 20 years of age, are wheelchair dependent with marked spinal deformity. ${ }^{9}$ Respiratory complications in FSH muscular dystrophy are therefore uncommon in childhood and more likely to occur in those with infantile onset.

\section{Emery-Dreifuss muscular dystrophy}

Emery-Dreifuss muscular dystrophy (EDMD) is a genetically heterogeneous condition. EDMD1 is $X$ linked due to mutations in the emerin gene; EDMD2 and EDMD3 are autosomal dominant and recessive conditions respectively due to mutations in the lamin $A / C$ gene. Other forms (EDMD4, 5 and 6 ) are caused by mutations in other genes (SYNE1, SYNE2 and FHL1). Presentation may be at any age, most commonly in late childhood or early adult life. There are early contractures at the elbows and Achilles tendons before there is significant weakness. Spinal rigidity llimited trunk and neck flexion) is another feature. Muscle weakness occurs in a scapulohumeroperoneal distribution with scapular winging, weakness of biceps and triceps and ankle dorsiflexion weakness. Cardiomyopathy with conduction defects and atrial arrhythmias can occur with emerin gene mutations while ventricular fibrillation and dilated cardiomyopathy are more common with lamin $\mathrm{A} / \mathrm{C}$ gene mutations. With lamin $A / C$ gene mutations there may be a very wide range in severity with severe cases having a congenital onset and the propensity for respiratory problems in childhood. $^{10}$

\section{Congenital muscular dystrophies}

In this group of conditions, presentation is at birth or in early childhood, with hypotonia, weakness and contractures. The conditions are static or show only slow progression. Creatine kinase may be normal or mildly raised, while muscle biopsy shows dystrophic changes. Many of these conditions have associated structural brain abnormalities and eye abnormalities.

Merosin-deficient congenital muscular dystrophy (CMD1A) is the most common of these conditions. It is a recessive condition caused by mutations in the lamin A2 gene leading to deficiency of laminin A2 (merosin) in the extracellular matrix of muscle. There is a spectrum of severity but mildly affected cases are less common than patients with severe weakness of face, trunk and limbs from birth. Feeding and respiratory difficulties are common. White matter changes are seen on MRI brain scan. Cognition is usually normal. Epilepsy occurs in $30 \%$.

Another group of congenital muscular dystrophies is due to disorders of glycosylation of $\boldsymbol{\alpha}$-dystroglycan which results in abnormal basal lamina formation in brain muscle and eye. These types of congenital muscular dystrophies are associated cortical dysplasia and eye abnormalities. Included are Fukuyama congenital muscular dystrophy, muscle-eye-brain disease and Walker-Warburg syndrome. These conditions are generally associated with learning difficulty, which may be so severe that the profound hypotonia and lack of movement may be attributed to the brain disorder and muscle involvement overlooked.

Ullich congenital muscular dystrophy is a disorder of collagen $\mathrm{VI}$, as is Bethlem myopathy, a milder disorder. In Ullrich congenital muscular dystrophy there is muscle weakness with proximal contractures and distal joint laxity. There may also be orthopaedic problems; hip dysplasia, torticollis and scoliosis. Bulbar muscles are generally not severely affected. Vital capacity declines from the middle of the first decade and around $70 \%$ require non-invasive ventilation in adolescence. ${ }^{11}$

\section{Rigid spine syndrome and rigid spine muscular dystrophy}

Rigid spine syndrome is characterised by early rigidity of the spine due to axial muscle contractures and is described in several neuromuscular disorders including EDMD, minicore myopathy and collagen VI myopathies. Because of ankylosis of the costovertebral joints and as there is often early diaphragmatic involvement in these conditions, respiratory insufficiency frequently occurs when limb weakness may be mild.

One form of rigid spine muscular dystrophy is caused by mutations in the selenoprotein N1 (SEPN1) gene. Mutations in this gene can also cause minicore myopathy, a desmin myopathy and congenital fibre type disproportion. Individuals with SEPN1 related myopathy appear to be at particular risk of early respiratory insufficiency. ${ }^{12}$ 


\section{Congenital myopathyies}

This is a group of muscle disorders with early onset of hypotonia and weakness. They tend to be stable or slowly progressive. The different types of congenital myopathy are defined by findings on muscle biopsy.

\section{Central core disease}

Characteristically there is hypotonia from infancy, delayed motor development and facial and proximal limb weakness. Skeletal deformities, congenital hip dislocation, scoliosis and foot deformity are common. Cores, central areas of derangement of sarcomeres with absence of oxidative enzyme activity, are seen on muscle biopsy. Mutations in the ryanodine receptor gene (RYR1) are found in the majority of patients. RYR1 mutations are also associated with malignant hyperthermia so patients with central core disease must be considered at risk of this anaesthetic complication. Inheritance is usually autosomal dominant but rarely may be recessive. Cardiomyopathy and respiratory insufficiency are rare.

\section{Minicore disease}

In this condition muscle biopsy shows multiple small areas devoid of oxidative enzyme activity. There is hypotonia, delayed motor development and weakness which is mainly proximal and axial. There are early contractures of paraspinal muscles with spinal rigidity and scoliosis. Diaphragmatic involvement occurs early with a risk of the development of respiratory insufficiency while independent ambulation persists.

\section{Nemaline myopathy}

This condition is characterised by the finding on muscle biopsy of multiple rod-like particles derived from Z band material. It is genetically heterogeneous with mutations in six genes currently reported. Inheritance may be recessive or dominant.

There is a severe neonatal form with respiratory insufficiency, marked hypotonia and weakness and bulbar insufficiency. Affected infants may be ventilator dependent from birth or have early respiratory complications. Milder forms with less severe muscle weakness may have selective diaphragmatic involvement and early respiratory insufficiency.

\section{Myotubular myopathy (centronuclear myopathy)}

The characteristic muscle biopsy finding is multiple central nuclei surrounded by a clear zone. There is a severe neonatal form which is $X$ linked and due to mutations in the MTM1 gene. Pregnancy with an affected fetus is usually complicated by polyhydramnios and reduced fetal movements. Affected neonates are hypotonic with ptosis and ophthalmoplegia and profound weakness and around $85 \%$ require ventilatory support. An international questionnaire study identified 116 affected individuals, of whom 63 had died (median age at death 2.5 months). Of the survivors (median age 4.5 years), $70 \%$ required full-time ventilatory support, $15 \%$ required support for $<12 \mathrm{~h}$ per day and $15 \%$ had achieved respiratory independence. ${ }^{13}$ In some families the clinical phenotype is less severe. Milder forms have autosomal recessive or dominant inheritance.

\section{Congenital fibre-type disproportion myopathy}

In this condition, the muscle biopsy shows type I muscle fibres with a smaller diameter than type II fibres. Onset is usually congenital or in childhood, and severity varies from mild to severe. Weakness involves the face, and proximal more than distal limbs; bulbar weakness and respiratory muscle weakness occur in about $30 \%$ of affected children. There is genetic heterogeneity; to date mutations have been described in three genes (ACTA1, SEPN1, TPM3) all of which are associated with other congenital myopathies. Mutations in the $\alpha$-actin gene (ACTA1) are associated with rapid progression and early respiratory failure, mutations in the SEPN1 gene are associated with spinal rigidity, relative preservation of limb strength and respiratory failure in adolescence or early adult life.

\section{Myotonic dystrophy}

\section{Myotonic dystrophy 1}

Myotonic dystrophy 1 is an autosomal dominant condition due to an expansion of a variable number of CTG repeats within the DMPK gene on chromosome 19q. Severity depends on the length of CTG expansion (normal 5-37, affected $>50$, congenital 2000). An increase in the size of the expansion tends to occur with maternal inheritance. This is a multisystem disorder causing muscle weakness and myotonia. Weakness selectively affects face, ptosis, bulbar muscles, neck flexion, trunk and distal limbs with proximal weakness occurring later in the course of the condition. Other features include cataract, diabetes, hypogonadism and cognitive effects. Congenital onset is often associated with respiratory insufficiency and feeding difficulty in the neonatal period which may improve. All have learning difficulty.

\section{Myotonic dystrophy 2 (proximal myotonic myopathy)}

This tends to be less severe than myotonic dystrophy 1. Muscle weakness is proximal rather than distal. Severely affected congenital cases do not occur.

\section{Congenital myasthenic syndromes}

Congenital myasthenic syndromes are genetic disorders of the neuromuscular junction in which the safety margin of neuromuscular transmission is compromised. Mutations may be found in genes for a number of components of the apparatus involved in neuromuscular transmission, but a proportion of cases remain genetically undefined as yet. These conditions are distinct from myasthenia gravis which is an acquired immunologically mediated disorder of the neuromuscular junction. Symptoms are often present in the neonatal period and include hypotonia, feeding difficulty, contractures and respiratory insufficiency. ${ }^{14}$ In some of these conditions there is ptosis and ophthalmoplegia. Congenital stridor, sometimes due to bilateral vocal cord paresis requiring intubation, may occur with Dok7 mutations. In these conditions weakness is exacerbated during intercurrent illnesses. With mutations in the rapsyn (RAPSN) and cholineacetyltransferase (CHAT) genes there is a particular propensity to episodic apnoea, especially during intercurrent illness.

\section{Mitochondrial myopathies}

Disorders of oxidative phosphorylation are frequently multisystem disorders and those presenting in early childhood are usually progressive causing early death. Isolated myopathy or neuropathy is uncommon. There are two conditions in which skeletal muscle is selectively affected which may cause respiratory failure in childhood Mutations in the thymidine kinase 2 gene (TK2) cause a mitochondrial DNA depletion syndrome which may manifest clinical variability. Some mutations may cause a rapidly progressive myopathy with respiratory failure in infancy. ${ }^{15}$ An unusual and benign cytochrome $c$ oxidase deficiency myopathy is also described. These infants have marked hypotonia and weakness with feeding and respiratory difficulties (which may require support with mechanical ventilation) in the first days or weeks of life with many of the features of other progressive mitochondrial disorders (raised plasma lactate, muscle biopsy showing ragged red fibres, and ultrastructurally abnormal mitochondria). Uniquely, this condition is associated with spontaneous improvement, which occurs between 5 and 20 months of age, with most children making a full recovery by 2 or 3 years of age. ${ }^{16}$ Genetic testing is available for this condition.

\section{Charcot-Marie-Tooth disease}

Genetic neuropathies are clinically and genetically heterogeneous. CharcotMarie-Tooth (CMT) disease is classified as CMT1 which is demyelinating or CMT2 which is axonal. Each of these is genetically heterogeneous. The terms congenital hypo-myelinating neuropathy and Djerine-Sotas neuropathy were used for severely affected infants; these may be caused by recessive or de novo dominant mutations. CMT4 denotes recessive neuropathies. The majority are slowly progressive and cause distal weakness and foot deformity with little or no respiratory involvement. Those severely affected in infancy or early childhood may develop respiratory failure. Mutations in the GDAP1 gene cause a neuropathy which may be either axonal or demyelinating, dominant or recessive. ${ }^{17}$ Recessive mutations in GDAP1 are particularly associated with vocal cord paresis and diaphragmatic weakness. ${ }^{18}$

\section{Pompe disease}

Pompe disease is a disorder of glycogen metabolism due to lysosomal acid $\alpha$ glucosidase deficiency. There is a spectrum of severity depending on the level of residual enzyme activity. Enzyme replacement therapy is now available. Infantile onset disease is characterised by cardiomyopathy, macroglossia and severe weakness of respiratory and skeletal muscle with $<10 \%$ ventilator-free survival rate at 18 months prior to the availability of treatment. ${ }^{19} \mathrm{~A}$ recent survey of infants treated with enzyme replacement therapy found an overall ventilator-free survival of $35 \%$, and ventilatordependent survival of $30 \% .{ }^{20}$ Juvenile and late onset disease causes progressive limb weakness and respiratory insufficiency either of which may present first. Earlier onset is associated with more rapid progression. Presentation may be with a rigid spine syndrome. Enzyme replacement therapy appears to stabilise the disease. ${ }^{21}$

\section{REFERENCES}

1. Dubowitz V. Chaos in the classification of SMA: a possible resolution. Neuromuscul Disord 1995:5:3-5.

2. Ioos C, Leclair-Richard D, Mrad S, et al. Respiratory capacity course in patients with infantile spinal muscular atrophy. Chest 2004;126:831-7.

3. Zerres K, Rudnik-Schöneborn S, Forrest E, et al. A collaborative study on the natural history of childhood and juvenile onset proximal spinal muscular atrophy (type II and III SMA): 569 patients. J Neurol Sci 1997:146:67-72.

4. Guenther UP, Varon R, Schlicke M, et al. Clinical and mutational profile in spinal muscular atrophy with respiratory distress (SMARD): defining novel phenotypes through hierarchical cluster analysis. Hum Mutat 2007;28:808-15. 
5. Eagle M, Baudouin SV, Chandler C, et al. Survival in Duchenne muscular dystrophy: improvements in life expectancy since 1967 and the impact of home nocturnal ventilation. Neuromuscul Disord 2002;12:926-9.

6. Kohler M, Clarenbach CF, Bahler C, et al. Disability and survival in Duchenne muscular dystrophy. J Neurol Neurosurg Psychiatry 2009;80:320-5.

7. Manzur AY, Kuntzer T, Pike M, et al. Glucocorticoid corticosteroids for Duchenne muscular dystrophy. Cochrane Database Syst Rev 2008;(1):CD003725

8. Biggar WD, Harris VA, Eliasoph L, et al. Long-term benefits of deflazacort treatment for boys with Duchenne muscular dystrophy in their second decade. Neuromuscul Disord 2006;16:249-55.

9. Wohlgemuth M, van der Kooi EL, van Kesteren RG, et al. Ventilatory support in facioscapulohumeral muscular dystrophy. Neurology 2004;63:176-8.

10. Mercuri E, Poppe M, Quinlivan R, et al. Extreme variability of phenotype in patients with an identical missense mutation in the lamin $A / C$ gene: from congenital onset with severe phenotype to milder classic Emery-Dreifuss variant. Arch Neurol 2004;61:690-4.

11. Nadeau A, Kinali M, Main $M$, et al. Natural history of Ullrich congenital muscular dystrophy. Neurology 2009;73:25-31.

12. Schara $\mathbf{U}$, Kress $\mathbf{W}$, Bonnemann $\mathrm{CG}$, et al. The phenotype and long-term follow-up in 11 patients with juvenile selenoprotein N1-related myopathy. EurJ Paediatr Neurol 2008;12:224-30.

13. McEntagart M, Parsons G, Buj-Bello A, et al. Genotype-phenotype correlations in X linked myotubular myopathy. Neuromuscul Disord 2002;12:939-46.

14. Kinali M, Beeson D, Pitt MC, et al. Congenital myasthenic syndromes in childhood: diagnostic and management challenges. J Neuroimmunol 2008;201-202:6-12.

15. Blakely E, He L, Gardner JL, et al. Novel mutations in the TK2 gene associated with fatal mitochondrial DNA depletion myopathy. Neuromuscul Disord 2008; 18:557-60.

16. Horvath R, Kemp JP, Tuppen HA, et al. Molecular basis of infantile reversible cytochrome c oxidase deficiency myopathy. Brain 2009;132:3165-74.

17. Moroni I, Morbin M, Milani M, et al. Novel mutations in the GDAP1 gene in patients affected with early-onset axonal Charcot-Marie-Tooth type 4A. Neuromuscul Disord 2009;19:476-80.

18. Sevilla T, Jaijo T, Nauffal D, et al. Vocal cord paresis and diaphragmatic dysfunction are severe and frequent symptoms of GDAP1-associated neuropathy. Brain 2008;131:3051-61.
19. Kishnani PS, Hwu WL, Mandel $\mathrm{H}$, et al. A retrospective, multinational multicenter study on the natural history of infantile-onset Pompe disease. $J$ Pediatr 2006;148:671-6.

20. Kishnani PS, Corzo D, Leslie ND, et al. Early treatment with alglucosidase alfa prolongs long-term survival of infants with Pompe disease. Pediatr Res 2009;66:329-35

21. Strothotte S, Strigl-Pill N, Grunert B, et al. Enzyme replacement therapy with alglucosidase alfa in 44 patients with late-onset glycogen storage disease type 2: 12-month results of an observational clinical trial. J Neurol 2010:257:91-7.

\section{APPENDIX 3}

\section{LIST OF STAKEHOLDERS}

- Action Duchenne

- Association of Chartered Physiotherapists in Respiratory Care

- Association for Glycogen Storage Disease (UK)

- Association for Palliative Medicine of Great Britain and Ireland

- Association of Paediatric Anaesthetists

- Association of Paediatric Chartered Physiotherapists

- Association of Respiratory Nurse Specialists

- Association for Respiratory Technology and Physiology

- British Myology Society

- British Paediatric Respiratory Society

- Jennifer Trust

- Muscular Dystrophy Campaign

- Primary Care Respiratory Society (UK)

- Royal College of Anaesthetists

- Royal College of General Practitioners

- Royal College of Nursing

- Royal College of Paediatrics and Child Health

- Royal College of Physicians

- Royal College of Physicians and Surgeons of Glasgow

- Royal College of Physicians, Edinburgh

- Speech and Language: Ms A Aloysius, Specialist Paediatric Speech and Language Therapist 